号

Review of Scientific and Technical Options for the Superconducting Super Collider Program

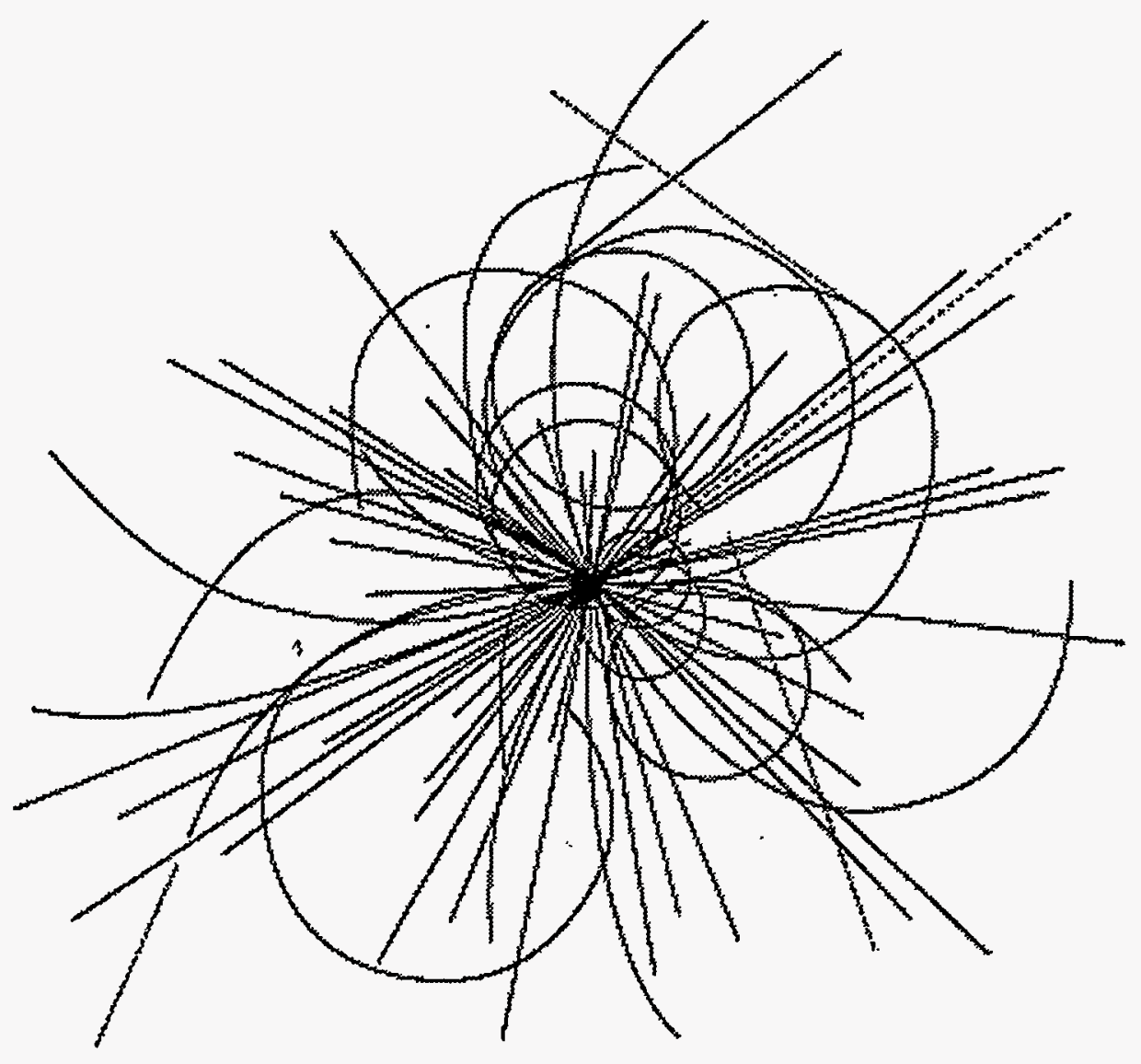

\section{Superconducting Super Collider Laboratory}

SSCL-SR-1230

November 1993

Distribution Category: $\mathbf{4 0 0}$

T. Dombeck
APPROVED FOR RELEASE OR PUBLIGATION - O.R. PATENYT GROUP

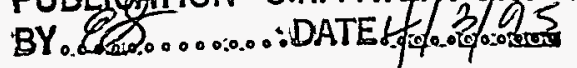




\section{Disclaimer Notice}

This report was prepared as an account of work sponsored by an agency of the United States Govemment. Neither the United States Govemment or any agency thereof, nor any of their employees, makes any warranty, express or implled, or assumes any legal liability or responsibility for the accuracy, completeness, or usefuiness of any information, apparatus, product, or process disclosed, or represents that its use would not intringe privately owned rights. Reference herein to any specific commercial product, process, or service by trade name, trademark, manufacturer, or othenwise, does not necessarily constitute or imply its endorsement, recommendation, or tavoring by the United States Government or any agency thereof. The views and opinions of authors expressed herein do not necessarily state or reflect those of the United States Govemment or any agency thereof.

Superconducting Super Collider Laboratory is an equal opportunity employer. 


\section{DISCLAIMER}

Portions of this document may be illegible in electronic image products. Images are produced from the best available original document. 
SSCL-SR-1230

\title{
Review of Scientific and Technical Options for the Superconducting Super Collider Program
}

T. Dombeck

\author{
Superconducting Super Collider Laboratory* \\ 2550 Beckleymeade Ave. \\ Dallas, TX 75237 USA
}

November 1993

*Operated by the Universities Research Association, Inc., for the U.S. Department of Energy under Contract No. DE-AC35-89ER40486. 


\title{
Review of Scientific and Technical Options
}

\author{
for the Superconducting Super Collider Program
}

Summary

This document is a review of options for the Superconducting Super Collider (SSC) Program. It is the result of an informal study by an ad-hoc working group consisting of Laboratory physicists and engineers who investigated the physics and technical implications of a number of possible alternative SSC programs. Previous studies have shown, and early in this study it was confirmed, that a collider of approximately $20 \mathrm{TeV}$ protons on $20 \mathrm{TeV}$ protons with a luminosity of $10^{33} \mathrm{~cm}^{-2} \mathrm{~s}^{-1}$ at each interaction region (with an upgrade path to $10^{34}$ ) is needed to support a physics program that is guaranteed to answer existing particle physics questions and make new discoveries. Therefore, all options considered in this document were consistent with attainment of these original goals for the SSC.

One promising option considered was a program of colliding anti-protons on protons as a possible means to reduce the cost of the SSC by eliminating one of the Collider rings. However, the luminosity requirements to obtain the SSC physics goals remains the same as for protons colliding with protons and this study confirms that even though progress has been made over the last ten years in obtaining the high intensity anti-proton beams necessary, a luminosity higher than $10^{32}$ cannot be guaranteed.

Other options were examined to see what advantages could be derived by departing from the SSC baseline program, either in schedule, in parameters, by staging, or by combinations of these options. Even though we considered re-examination of the cost of the baseline program to be beyond the scope of this document, differential cost savings were estimated. Finally, a brief survey of progress over the last ten years in various technical areas that might lead to more cost effective engineering designs was included in this study, such as higher magnetic field magnets resulting from lower operating temperatures or higher current-carrying superconducting materials. It was determined that the technology in these areas had not advanced significantly to warrant redesign of SSC components. 


\section{Table of Contents}

1. Introduction

1.1. SSC Baseline Design

1.2. Options considered

1.3. References

2. Physics Considerations

2.1. Introduction

2.2. High $P_{t}$ Physics Processes

2.2.1.Electroweak Symmetry Breaking

2.2.2.Intermediate Mass $\mathrm{H} \rightarrow \boldsymbol{\gamma}$

2.2.3.Intermediate Mass $\mathrm{H} \rightarrow Z Z^{*}$

2.2.4.Heavy-mass Higgs

2.2.5.Physics of the Top Quark

2.2.6.SUSY Searches in Gluino Pairs

2.2.7. Heavy Guage Vector Boson Searches

2.2:8. Excited Quark Searches

2.3. Accelerator Limitations

2.4. References

3. Proton-Antiproton Collider at $20 \times 20 \mathrm{TeV}$

3.1. Program Summary

3.2. Physics Program

3.2.1. Overview

3.2.2.Detector Issues

3.3. Relationship to Other Programs

3.4. Machine Configuration

3.4.1.The Antiproton Source

3.4.2.Luminosity

3.4.3. Collider Issues

3.4.4.Beam Separation

3.5. Conclusion

3.6. Cost Estimate

3.7. References

4. Missing Coil or Missing Magnet Schemes for $p-p$ collider at $10 \times 10 \mathrm{TeV}$

4.1. Program Summary (Missing Coils)

4.2. Physics Program

4.3. Relationship to Other Programs

4.4. Cost/Schedule Summary

4.5. Program Summary (Missing Magnets)

4.6. Physics Program

4.7. Relationship to Other Programs

4.8. Cost Summary 
5. Asymmetric $p-p$ collider at $2 \times 20 \mathrm{TeV}$

5.1. Program Summary

5.2. Physics Program

5.3. Detector for Asymmetric Collider

5.4. Cost/Summary

6. P-p collider at $2 \times 2 \mathrm{TeV}$

6.1. Program Summary

6.2. Physics Program

6.3. Relationship to Other Programs

6.4. Cost/Schedule Summary

7. Asymmetric $p-p$ collider at $0.2 \times 2 \mathrm{TeV}$

7.1. Program Summary

7.2. Machine Configuration

7.3. Physics Program

7.4. Physics Potential of MEB-HEB Collider

8. $2 \mathrm{TeV}$ Fixed Target Program

8.1. Program Summary

8.2. Physics Program

8.3. Relationship to Other Programs

8.4. Test Beam Program for Detector Development at $2 \mathrm{TeV}$

8.5. Cost/Schedule Summary

8.6. References

9. Single collider ring with fixed target at $20 \mathrm{TeV}$

9.1. Introduction and Summary

9.2. Beam Parameters

9.3. Physics of Heavy Flavor Particles

9.4. Experimental Facilities and Detectors

9.5. Summary

9.6. References

10. Technical Advances

10.1.High-field magnet

10.2.High Temp Superconductors

10.3.Artificial Pinning Centers In Superconducting Fibers

10.4.Cryogenic Refrigeration

Appendix A. Working Group Members

Appendix $B$. Assigned Section Editors

Appendix C. The Text of the 1986 P-Bar-P Study Group's Conclusions 


\section{Introduction}

Current funding difficulties facing the SSC Program suggest strongly that a spectrum of options for reaching the $20 \times 20 \mathrm{TeV}$ pp Collider be identified. This report has as its purpose to identify and explore in a preliminary way, possible options for such a program. The purpose is not to rank or judge these possibilities but to identify and characterize them in a useful way so that later inquiries demanding the production of such studies will have a good starting point and an idea of the general benefits and costs of the options that have been identified.

It should also be understood that this work constitutes only an informal study and should not be interpreted as having any offical status in the SSC Program. Rather, it should be identified with the physics research activity of the SSCL physics and technical community whose normal mission includes the ongoing study of the physics potential of the laboratory plus possible physics advantages or benefits that could be realized by means of modification of the official laboratory program.

Likewise, the cost and schedule aspects are recognized to have the character of scaling numbers that could be developed into actual cost estimates provided that proved desirable. We should guard against attempts to represent the very preliminary numbers in this report as reliable bases for any official development of the concepts presented here.

After consideration of SSC physics goals, the fundamental guideline used in this study is that the final goal is a proton-proton collider at greater than $15 \mathrm{TeV}$ on $15 \mathrm{TeV}$, with a capability of luminosity of $10^{34} \mathrm{~cm}^{-1} \mathrm{~s}^{-1}$.

\subsection{SSC Baseline Design}

The SSC baseline design consists of five elements: the LINAC, the LEB, the MEB, the HEB, and the two-ring Collider. Four interaction regions, two of them high luminosity, and a test beam area using the MEB beam, are planned. The layout is shown in figure 1, [1] and some relevant machine parameters are given in Table 1.1. Note that the vertical separations of the machines are in part dictated by the desire to have effective shielding so that one machine can be operated while another machine is being accessed by personnel. While the HEB extraction straight section is vertically above the Collider, the MEB is displaced horizontally. Some of the options considered in this study might require rearrangement of this physical layout.

Table 1.1: Summary of SSC baseline design parameters

\begin{tabular}{|c|c|c|c|c|c|}
\hline Machine (mode) & $\begin{array}{c}\text { Peak } \\
\text { Momentum }\end{array}$ & $\begin{array}{c}\text { No. } \\
\text { particles }\end{array}$ & $\begin{array}{c}\text { Emittance } \pi \\
\text { mm-mrad }\end{array}$ & $\begin{array}{c}\text { Cycle } \\
\text { time } \\
(\mathbf{s})\end{array}$ & $\begin{array}{c}\text { Relative } \\
\text { elevation } \\
(\mathbf{m})\end{array}$ \\
\hline Collider & $20 \mathrm{TeV} / \mathrm{c}$ & $1.3 \times 10^{14}$ & 1.0 & -- & -46 \\
\hline HEB (collider) & $2 \mathrm{TeV} / \mathrm{c}$ & $2 \times 10^{13}$ & 0.8 & 120 & -32 \\
\hline MEB (collider) & $200 \mathrm{GeV} / \mathrm{c}$ & $8 \times 10^{12}$ & 0.7 & 3 & 0 \\
\hline MEB (test beam) & $200 \mathrm{GeV} / \mathrm{c}$ & $4 \times 10^{13}$ & 4 & 4 & 0 \\
\hline LEB (collider) & $12 \mathrm{GeV} / \mathrm{c}$ & $1 \times 10^{12}$ & 0.6 & 0.1 & 0 \\
\hline LINAC (collider) & $1.2 \mathrm{GeV} / \mathrm{c}$ & $1 \times 10^{10}$ & 0.5 & 0.1 & 0 \\
\hline
\end{tabular}

In the SSC baseline program, the civil construction of the collider tunnel is completed in 1996 with the magnet manufacture beginning 1994 and being completed 1998. The collider is commissioned 1999. The HEB is constructed beginning 1996 and commissioned in 1998. The MEB is commissioned 1996, with the LEB going into operation 1996 and the LINAC operating 
from 1995. The test beam program would be based only on the MEB and would begin 1996. The essence of the baseline is that collider construction proceeds concurrently with construction of the injectors. Two major detectors would be commissioned at the same time as the collider.

\subsection{Options considered}

The following were the ground rules under which options were considered:

- All options must be consistent with a final goal of greater than 15 on $15 \mathrm{TeV}$ at a luminosity of $10^{33} \mathrm{~cm}^{-2} \mathrm{~s}^{-1}$.

- The existing collider tunnel is to be used.

- Any new land acquisition or impact statement must not stretch out the schedule.

- The option should have an operating physics program by the year 2002 .

The options considered are summarized by category below.

\subsubsection{Schedule}

In this category, a stretched-out program changes the funding profile and may allow operations for test beams, while the physics program is essentially delayed until collider operations begin.

1. Stretched-out baseline. Work on the injectors is halted while work on the collider proceeds. There is no physics program.

2. Collider delayed, injectors completed (Rainer Meinke [2]). The physics program would consist of a test beam program for detector development at $200 \mathrm{GeV}$ and $2 \mathrm{TeV}$ or a fixed target program at 2 (and/or 20) TeV.

\subsubsection{Staging}

In this category, a physics program is begun before completion of the collider. The options are ordered roughly by CM energy, they include both fixed target and collider programs.

\subsubsection{Parameter changes}

In this category we considered technical advances that may have occurred over the last decade that may reduce overall collider costs if included in the design.

\subsubsection{Cost}

Options were costed in FY94 dollars (escalated by 1.03 x over FY93 dollars). Only the cost differential savings or cost increases beyond the baseline program are quoted.

\subsubsection{Summary}

Given the baseline high-energy machines (200 GeV MEB, $2 \mathrm{TeV} H E B, 20 \mathrm{TeV}$ collider), the appropriate options are listed in Table 1.2. 
Table 1.2. Options considered

\begin{tabular}{|l|c|c|c|c|}
\hline \multicolumn{1}{|c|}{ Description } & $\begin{array}{c}\text { CM } \\
\text { energy } \\
(\mathrm{TeV})\end{array}$ & $\begin{array}{c}\text { Luminosity } \\
\text { /flux }\end{array}$ & $\begin{array}{c}\text { Year } \\
\text { operational }\end{array}$ & $\begin{array}{c}20 \times 20 \mathrm{TeV} \text { pp } \\
\text { Operational }\end{array}$ \\
\hline Streched-out pp $20 \times 20 \mathrm{TeV}$ & 40 & $10^{33}$ & 2002 & 2002 \\
\hline Pbar-p 20 $20 \mathrm{TeV}$ & 40 & $10^{32}$ & 2000 & $? ?$ \\
\hline Missing magnet, $10 \times 10 \mathrm{TeV}$ & 20 & & & \\
\hline Missing coil, $15 \times 15 \mathrm{TeV}$ & 30 & & & $? ? ?$ \\
\hline Asymmetric $2 \times 20 \mathrm{TeV}$ & 13 & $10^{32}$ & 1999 & \\
\hline $3 \times 3 \mathrm{TeV}$ & 6 & & & \\
\hline $2 \times 2 \mathrm{TeV}$ & 4 & & & \\
\hline $0.2 \times 2 \mathrm{TeV}$ & 1.3 & & & \\
\hline $2 \mathrm{TeV}$ fixed target & 0.09 & & & \\
\hline $20 \mathrm{TeV}$ fixed target & 0.27 & & & \\
\hline
\end{tabular}

\subsection{References}

[1] "Site-specific Conceptual Design of the Superconducting Super Collider," J.R. Sanford and D.M. Matthews, editors, SSCL-SR-1056 (July 19, 1990)

[2] "Proposal for Rephasing the Superconducting Super Collider, Revision A," Rainer Meinke, July 1993

\section{Physics Considerations}

Previous studies [1] have shown that, in the multi- TeV region, the primary consideration affecting physics reach is luminosity and detector design (coverage in eta and resolution). For lower CM energies, say $5 \mathrm{TeV}$ or less, particle type becomes important since pair production from pbar-p collisions become important.

\subsection{Introduction}

In order to estimate the "physics reach" of various pp or p p colliders, several physics processes of interest have been studied. For each process, the number of produced events required to carry out meaningful physics studies or detect statistically significant signals has been estimated. These estimates are largely based on detailed studies for the SDC and GEM design reports. Extrapolation to other energies takes into account calculated cross sections and crude background estimates. To convert the required number of events into an average luminosity to be delivered by the machine, a "running year" of $10^{7}$ seconds is used. This time is consistent with SSC design plans, which call for an $80 \%$ detector up time and enough stable beam time to result in $10^{7}$ "live" seconds per operating year. Note that when making comparisons with existing facilities, any deviation from these assumptions should be taken into account.

\subsection{High $\mathbf{P}_{\mathrm{t}}$ Physics Processes}




\section{Introduction}

In order to estimate the "physics reach" of various $p p$ or $\bar{p} p$ colliders, several physics processes of interest have been studied. For each process, the number of produced events required to carry out meaningful physics studies or detect statistically significant signals has been estimated. These estimates are largely based on detailed studies for the SDC and GEM design reports. Extrapolation to other energies takes into account calculated cross sections and crude background estimates. To convert the required number of events into an average luminosity to be delivered by the machine, a "running year" of $10^{7}$ seconds is used. This time is consistent with SSC design plans, which call for an $80 \%$ detector up time and enough stable beam time to result in $10^{7}$ "live" seconds per operating year. Note that when making comparisons with existing facilities, any deviation from these assumptions should be taken into account. The conclusions of this study are generally similar to those reached by Eichten, et al. [2] in 1984 and by the Drell Panel [3] in 1990.

\subsubsection{Electroweak Symmetry Breaking}

Present indications are that experiments at LEP-II will most likely be capable of discovering the standard-model Higgs boson up to a mass region of roughly $80 \mathrm{GeV} \mathrm{[4].}$ Experiments at hadron colliders must therefore be prepared to extend the search for the Higgs boson from $80 \mathrm{GeV}$ on upwards. The theoretical upper limit is about $650 \mathrm{GeV}$ but might be stretched to $800 \mathrm{GeV}$. While the production cross sections are large, $1-100 \mathrm{pb}$, it is necessary to rely on rare decay modes to overcome backgrounds. The most favorable modes are $H \rightarrow \gamma$ for $80<M_{H}<130 \mathrm{GeV}, H \rightarrow Z Z^{*} \rightarrow l^{+} l^{-} l^{+} l^{-}$for $130 \mathrm{GeV}<M_{H}<$ $2 M_{Z}$, and $H \rightarrow Z Z \rightarrow l^{+} l^{-} l^{+} l^{-}$for $M_{H}>2 M_{Z}$. 


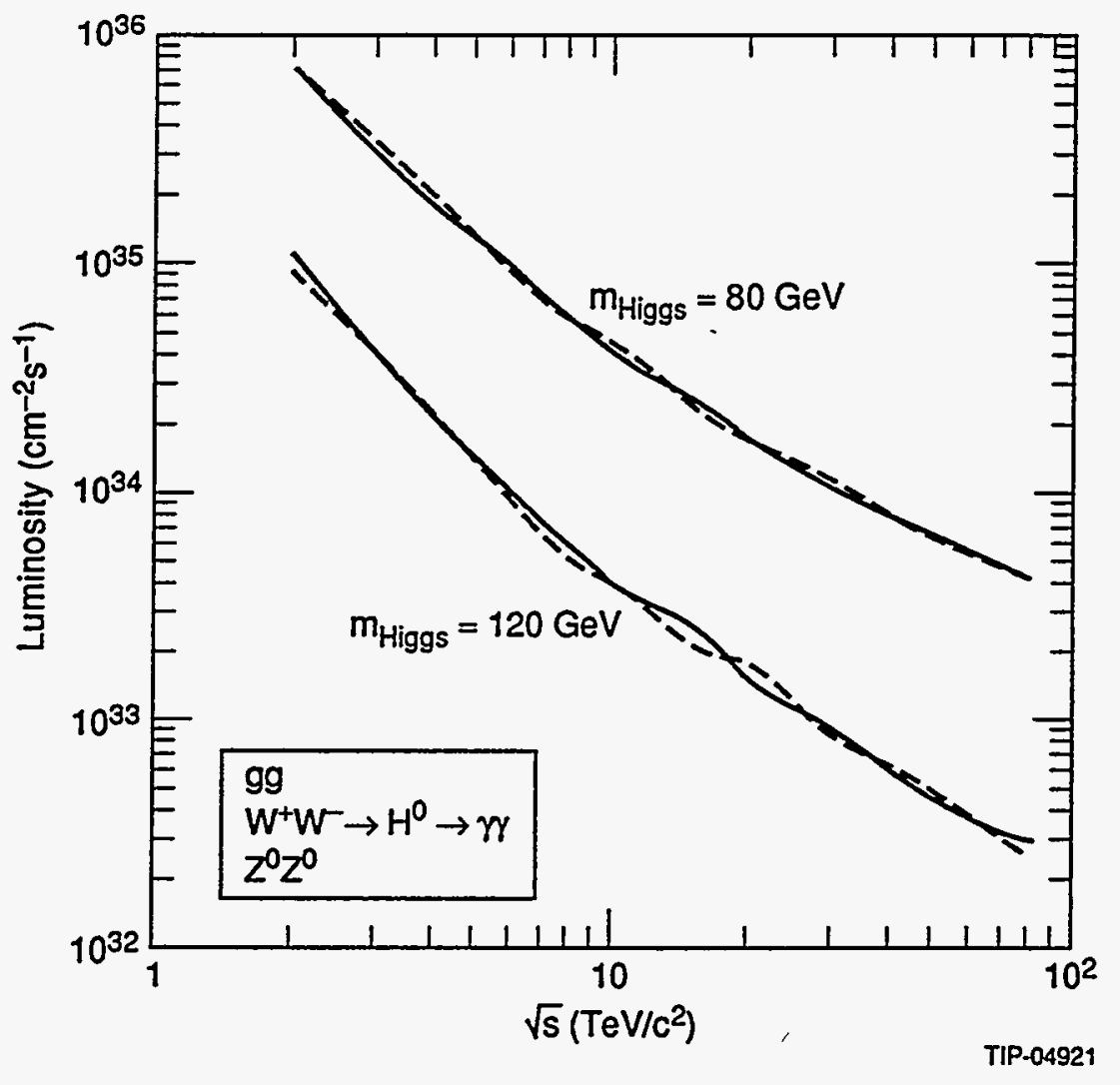

Fig. 1a. The instantaneous luminosity required to directly produce Higgs boson events such that the significance (signal $/ \sqrt{\text { background }})=5$. The Higgs boson events are required to decay via the mode $H^{0} \rightarrow \gamma$. Any increase in the QCD background at higher luminosity is not included. 


\subsubsection{Intermediate Mass $\mathrm{H} \rightarrow \gamma \gamma$}

Strategies to discover the Higgs boson in the mass range $80 \mathrm{GeV}<M_{H}<130 \mathrm{GeV}$ have been discussed in cases where the Higgs boson is directly produced (predominantly by gluon-gluon fusion) and also when it is produced in association with a $W$ boson or $\overline{t t}$ pair. In both cases, studies have focused on the decay $H^{0} \rightarrow \gamma$. Despite the relatively small branching ratio for this process $\left(\sim 10^{-3}\right)$, this mode is thought to be the most promising because it does not suffer from large QCD backgrounds for the dominant mode, $H \rightarrow b \bar{b}$. In the case of direct production, large residual backgrounds from $\overline{q q} \rightarrow \gamma \gamma, g g \rightarrow \gamma \gamma$, and $\pi^{0} \rightarrow \gamma$ from QCD two jet events still exist and will impose stringent requirements on the photon detection and $\pi / \gamma$ rejection capabilities of the detector. Extremely fine mass resolution will indeed be required to isolate a Higgs mass peak over this large continuum background. The results for direct production are based on a study presented in the GEM Technical Design Report [5]. The curves in Fig. 1a reflects the instantaneous luminosity required for a given $\sqrt{s}$ such that the significance is:

$$
S=\operatorname{signal} / \sqrt{\text { background }}=5 \text {. }
$$




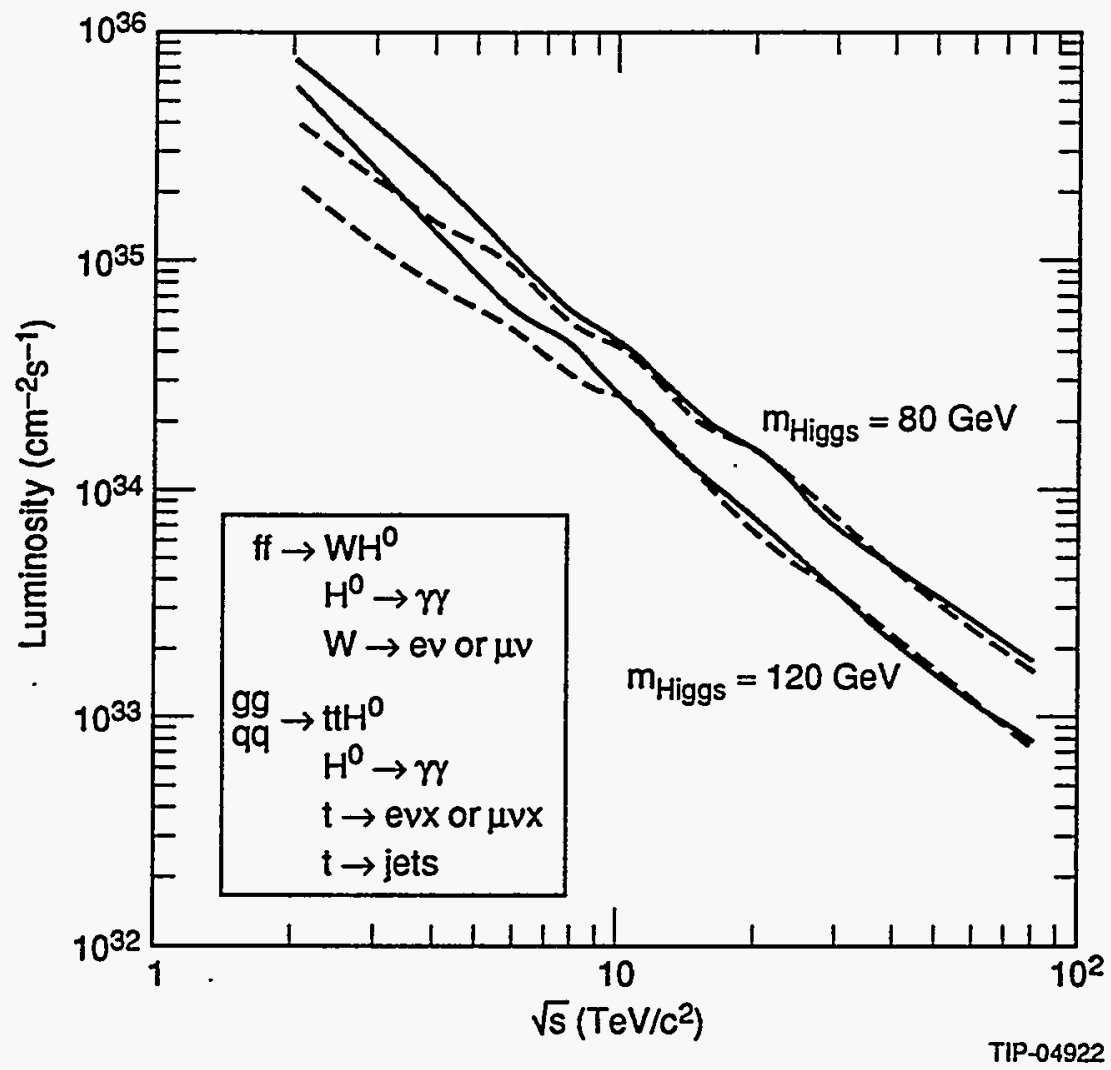

Fig. 1b. The instantaneous luminosity versus $\sqrt{s}$ for the associated production of Higgs boson in the modes $W H^{0}$ and $t H^{0}$. The curves reflect the luminosity required to product sufficient numbers of Higgs bosons such that the significance (signal $/ \sqrt{\text { background }}=5$ ) The Higgs bosons are required to decay via $H^{0} \rightarrow \gamma$ in both cases. The W boson decays leptonically into $e V$ or $\mu v$. In $t t H^{0}$ events one top quark decays semi-leptonically to produce an electron or muon and the other decays to jets. 
It should be mentioned that the background was calculated only for $\sqrt{s}=40 \mathrm{TeV}$. Since the predominant background is $q \bar{q} \rightarrow \gamma$, the ratio of signal to background will worsen as the center of mass energy is lowered. Therefore, the curves in Fig. 1a are overly optimistic at smaller $\sqrt{s}$.

The associated Higgs production mode cross sections for the processes $W H^{0}$ and $\bar{t} t H^{0}$ suffer by a reduction in nearly an order of magnitude relative to direct production. However, the requirement of a final state $l^{+}+\gamma \gamma+X$ significantly reduces backgrounds compared to only searching for di-photons. This final state therefore requires less photon mass resolution and $\gamma / j e t$ discrimination [6]. The major backgrounds to this processes are $W+\gamma \gamma$ and $Q Q+\gamma+$ jet and $Q Q+2$ jets. The results for the associated production modes are based on a study presented in the SDC Technical Design Report [7]. The curves in Fig. $1 \mathrm{~b}$ reflects the instantaneous luminosity required for a given $\sqrt{s}$ such that the significance $S=5$. This calculation of the significance was performed using Gaussian statistics, which is marginally correct when considering the numbers of events used (for example in the $80 \mathrm{GeV}$ Higgs case, there are 11 signal and an estimated 16 background events detected) but does overestimated the significance somewhat. In addition, a similar study presented in the GEM TDR [4],[8], shows a much lower efficiency for detecting signal events when using a more "realistic" detector simulation. However, it is felt the GEM study over-estimates the amount of background, since a significant part of the background comes from $Z \gamma$ events, and it should be possible to veto many of these.

\subsubsection{Intermediate Mass $H \rightarrow Z Z$ *}

The decay mode for the Higgs boson: $H^{0} \rightarrow Z Z^{*} \rightarrow(e e)(\mu \mu)$ is the favored mode to search in the mass range $130 \mathrm{GeV}<\mathrm{M}_{\mathrm{H}}<180 \mathrm{GeV}$, where the " $Z$ " stands for a virtual $Z$. In this mass range, the Higgs boson width remains quite narrow. A study of the production cross section times the branching ratio has been performed using the PYTHIA [8] generator with the $g g$ and WWIZZ fusion processes for the Higgs production. The allowed phase space correctly takes into account the $Z Z^{*}$ process.

The cross sections are calculated for both $p p$ and $\bar{p} p$ as a function of the center-of-mass energy, and the corresponding luminosity to produce 40 events per SSC year for these cross sections are shown in Fig. 2. A top quark mass of $m_{t}$ of $150 \mathrm{GeV}$ has been used. Systematic errors on the values of the cross section are mainly due to the structure functions $(\sim 30 \%)$ and the top quark mass $(\sim 15 \%)$. Close to the SSC in energy and luminosity are required to observe this mode for low masses or for $M \sim 170 \mathrm{GeV}$. 


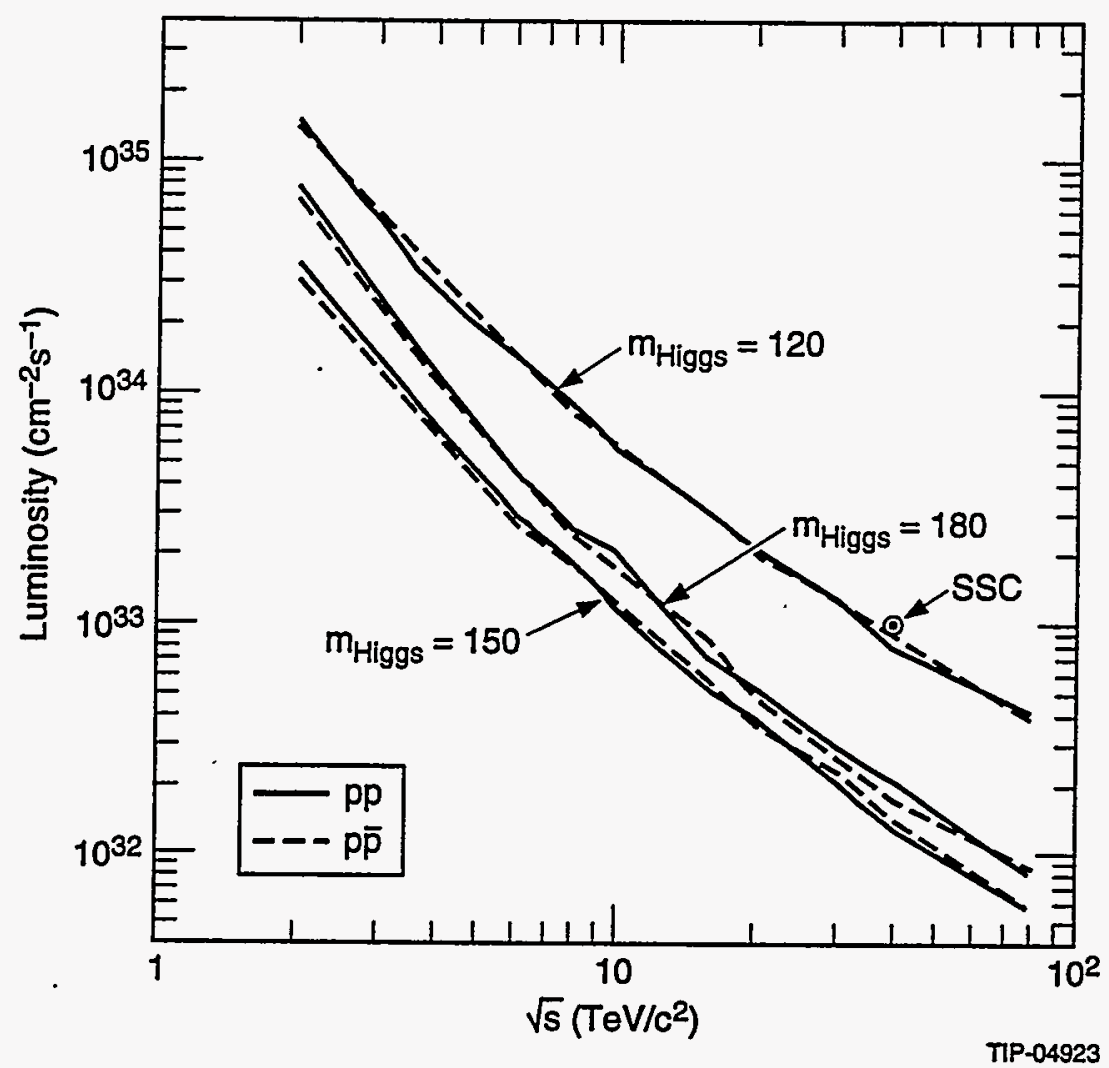

Fig. 2. The curves indicate the luminosity needed to produce 40 events/year in the mode $H \rightarrow Z Z \rightarrow 4 l(e$ or $\mu)$ in $p p$ collisions for Higgs masses of 200,600 , and $800 \mathrm{GeV}$. Curves for $\bar{p} p$ collisions (not shown) are similar. 


\subsubsection{Heavy-mass Higgs}

Figure 3 shows the luminosity needed to produce 20 events/year in the mode $H \rightarrow Z Z \rightarrow$ $4 l$ ( $e$ or $\mu$ only) in $p p$ collisions for Higgs masses of 200,400 and $800 \mathrm{GeV}$. The 20 event limit provides a reasonable estimate of the reach for this mode, based on SDC studies ${ }^{6}$ at $40 \mathrm{TeV}$ and $10^{33} \mathrm{~cm}^{-2} \mathrm{sec}^{-1}$ luminosity. The curves for $\bar{p} p$ collisions are similar to these. 


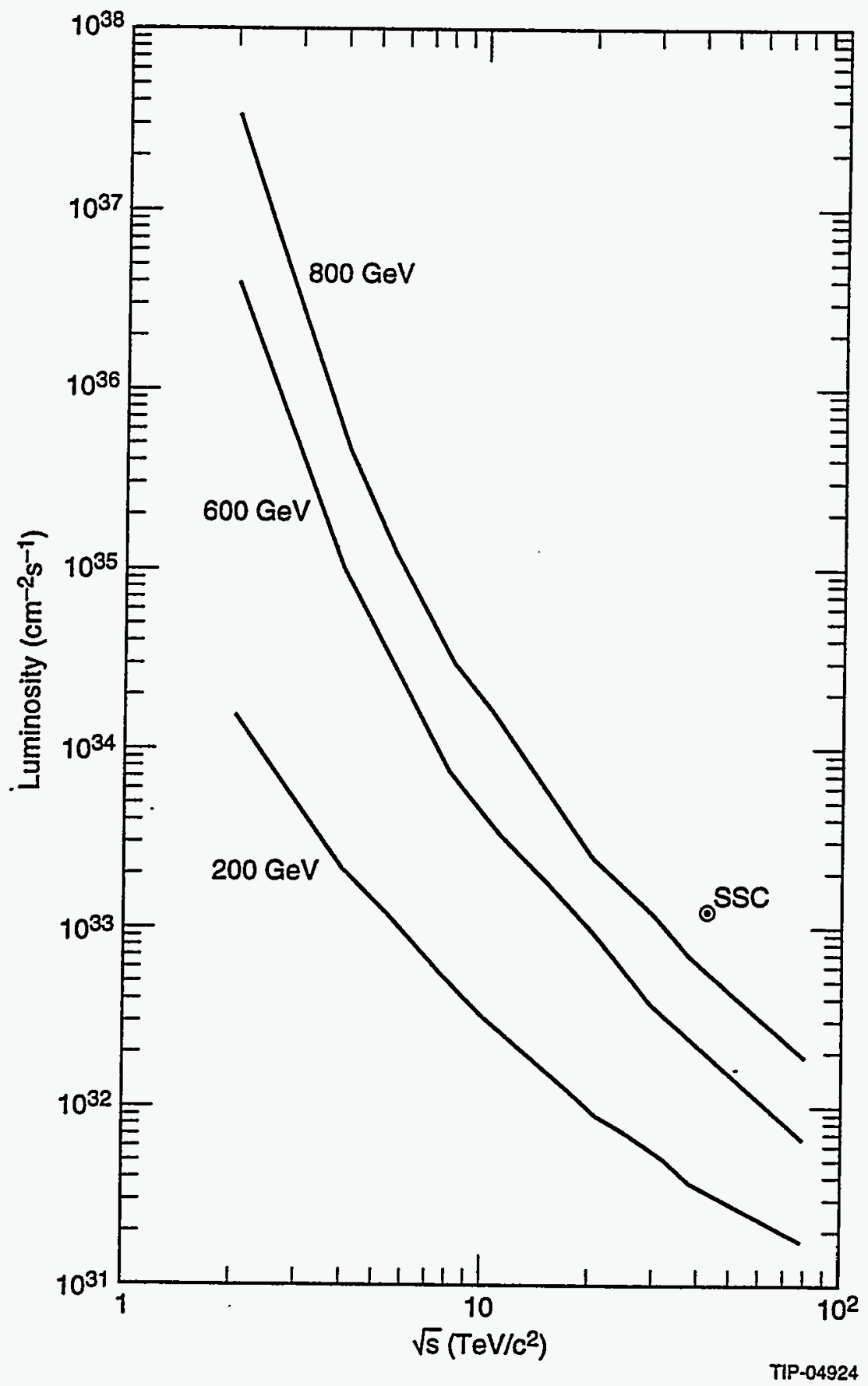

Fig. 3. The curves indicate the luminosity needed to produce 20 events/year in the mode $H \rightarrow Z Z \rightarrow 4 l$ (e or $\mu$ in $p p$ collisions for thiggs masses of 200,600 , and $800 \mathrm{GeV}$. Curves for $\bar{p} p$ collisions (not shown) are similar. 
This plot provides a conservative estimate of the reach attainable for the heavy Higgs. Note that use of the $\tau$ decay modes for one of the two $Z$ 's can increase the event sample by up to a factor of two. Also, for the highest masses decays of one of the two $Z$ s into neutrinos can be used, as was shown in the SDC and GEM detector Technical Design Reports [5], [7].

\subsubsection{Physics of the Top Quark}

The top quark is one of the two particles in the standard model that have not been observed (the other being the Higgs boson). It is presently thought that the top mass lies between 100 and $200 \mathrm{GeV}$, assuming that there is no new physics affecting higher order corrections to precision electroweak measurements. If the top mass is in this range, it will most likely be discovered at the FNAL Tevatron collider in the next few years. If it is not discovered there, it would almost certainly be discovered at any collider with reasonable luminosity and energy several times the collider due to the rapid rise in the top cross section with center-of-mass energy (Fig. 4).

Even if the top is discovered at the Tevatron, the rapid rise in the cross section indicates that many more top events would be produced in a higher energy machine, enabling better study of the properties of top. It is important to ask what properties of top are important to measure and how well can they be measured as a function of the machine energy and luminosity. The following properties were considered: (1) total cross section, (2) top mass, (3) observation of $t \rightarrow H^{+} b$ (where $H^{+}$is a charged Higgs), (4) branching ratios in the decay of the $W$ from the top, (5) flavor changing neutral current decays (such as $t \rightarrow Z+c)$, (6) angular distribution in $t \rightarrow W+b$, (7) polarization of the $W$ from top decay, and (8) rate of $t+\bar{t}+X$ as background to Higgs physics signatures at the SSC. Most of these (1, 4, 6 and 7) are probably well predicted by the standard model and unlikely to yield new physics or improved understanding of the standard model. Flavor changing neutral currents at a measurable level also seem unlikely and are probably ruled out by present limits. The decay $t \rightarrow W+s$ is very interesting but is probably impossibly difficult at the expected very small branching ratio and will not be considered further.

A precise measurement of the top mass is interesting because it is coupled with precise measurements of the masses of the $W$ and $Z$ and of other electroweak parameters through higher-order electroweak corrections. This gives a test of the consistency of the standard model and a deviation from expectation could indicate new physics. These higher-order corrections also depend logarithmically on the Higgs mass, so that a precise measurement of the top mass would give an indication of the Higgs mass. To predict the Higgs mass within a factor of two would require an error on the top mass of roughly $3 \mathrm{GeV}$. The SDC Technical Design Report [7] considers three methods of determining the top mass: lepton spectra in $e-\mu$ events, lepton spectra in sequential semileptonic decays $(t \rightarrow W+$ $b \rightarrow l+v+c+l+v)$ and invariant mass plots in three jet decays of top. The conclusion is that to achieve a statistical error on the top mass of $3 \mathrm{GeV}$ requires roughly 20000 top events when account is taken of branching ratios, acceptances, and efficiencies. Note that the SDC TDR estimates the systematic error tobe $3 \mathrm{GeV}$. 


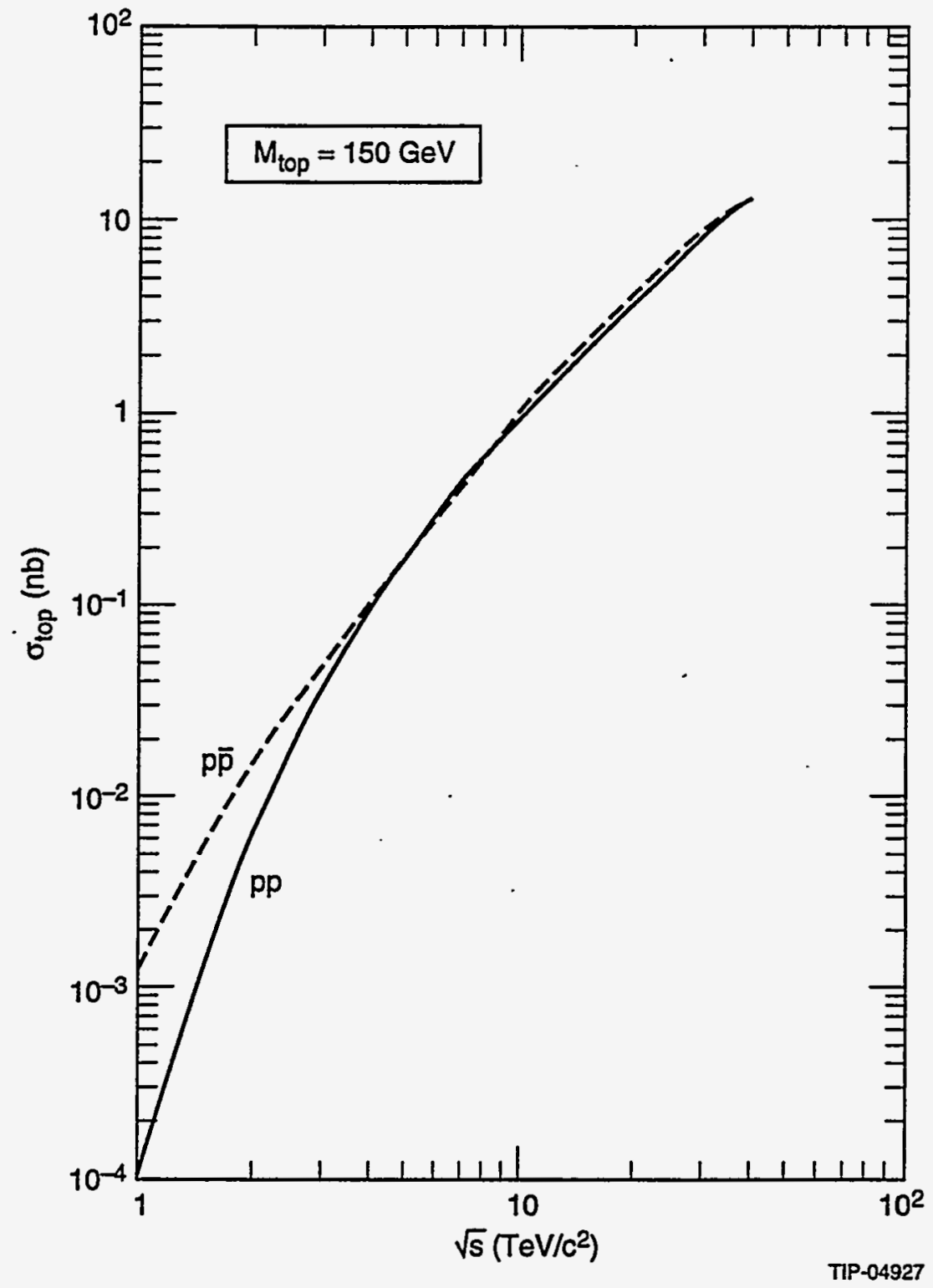

Fig. 4. Top cross section as a function of center-of-mass energy for $m_{t}=150 \mathrm{GeV}$ as calculated by PYTHIA using HMRSB structure functions. 
Figure 5a shows the luminosity required to produce this number of top events as a function of the center-of-mass energy. A pp machine with a center-of-mass energy of 4 $\mathrm{TeV}$ would need a luminosity of $2 \times 10^{31} \mathrm{~cm}^{-2} \mathrm{sec}^{-1}$. Any higher energy machine would do this easily. Also note that it is also likely that this precision will be obtained at the Tevatron $E_{\mathrm{cm}}=2 \mathrm{TeV}$ ) after the FNAL Main Injector upgrade (luminosity $=10^{32} \mathrm{~cm}^{-}$ $\left.{ }^{2} \sec ^{-1}\right)$. The exact reach does depend on the actual top mass.

If a charged Higgs exists with mass less than the top mass, it would be an extremely interesting signature to look for. This, however, is a difficult signature to uncover and is complicated by the fact that the $t \rightarrow H^{+} b$ branching ratio and the branching ratios of the various decay modes of the charged Higgs are model dependent. This signature was studied in the SDC TDR [7]. From the work there, it is estimated that 30 million top events are required to obtain at least a five standard deviation effect for $\left(m_{t}-m_{H^{+}}\right) \geq 25$ $\mathrm{GeV}$ and most choices of the other parameters. Figure $5 \mathrm{~b}$ shows the luminosity required to produce this number of top events as a function of the center-of-mass energy. As can be seen, this physics requires a machine with approximately the reach of the SSC, as is typical of most Higgs signatures. It should be noted that for parts of the multiple Higgs doublet parameter space the luminosity required to observe the $t \rightarrow H^{+} b$ decay is significantly less. 


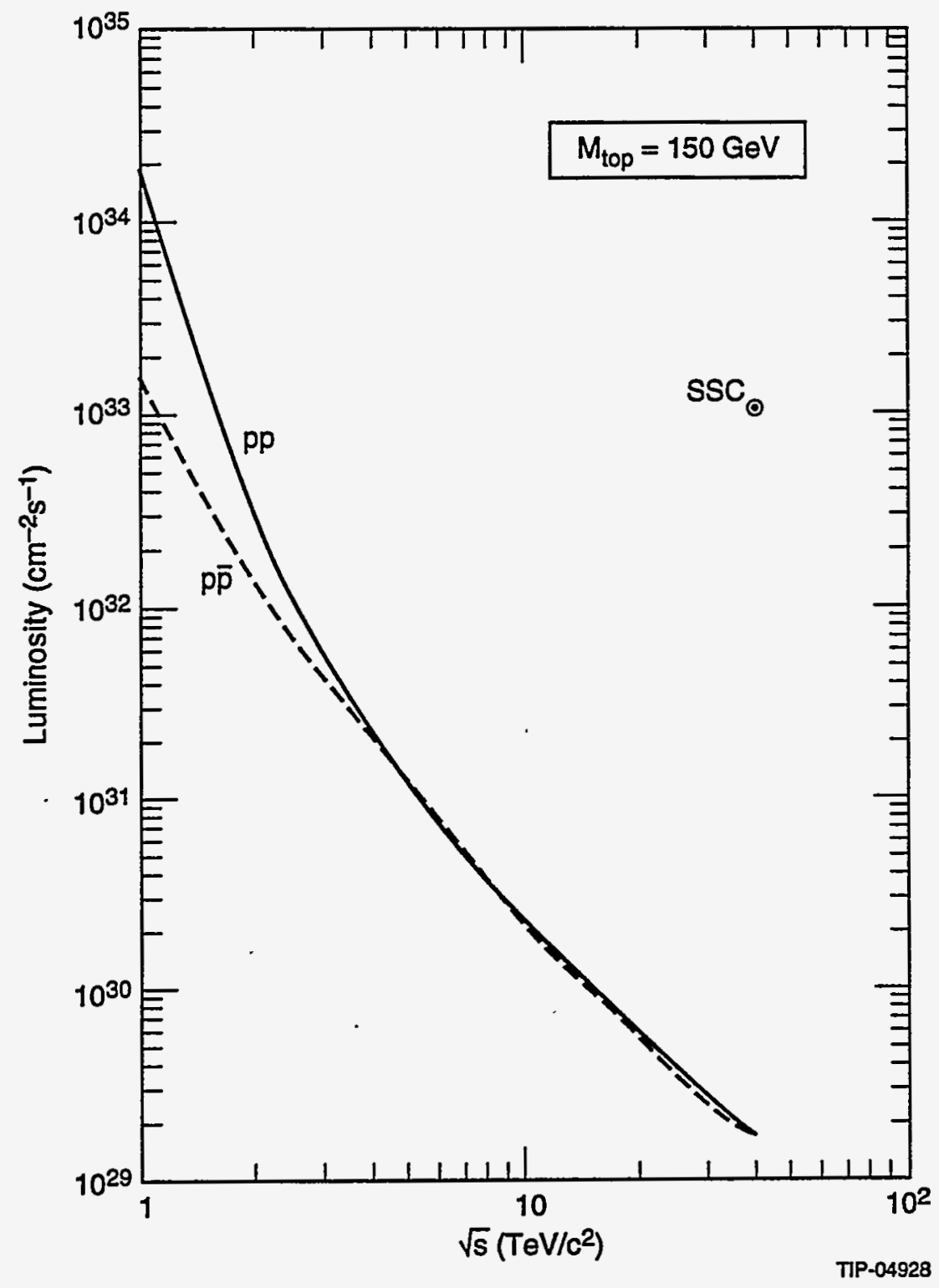

Fig. 5a. Instantaneous luminosity needed to produce 20000 it events as a function of $\sqrt{s}$. This would allow a measurement of the top mass to about $3 \mathrm{GeV}$. 


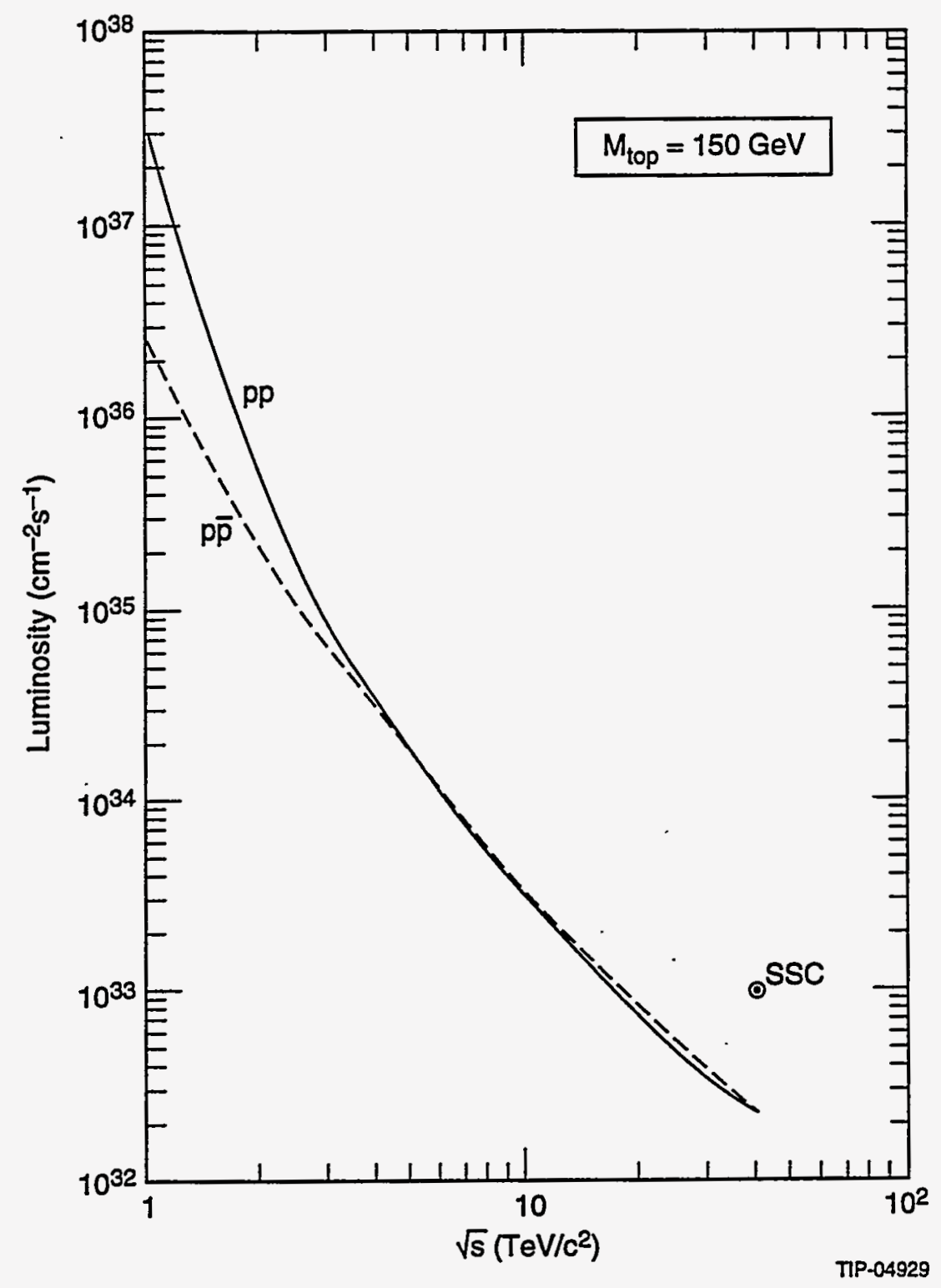

Fig. 5b. Instantaneous luminosity needed to produce $3 \times 10^{7}$ tt events as a function of $\sqrt{s}$. This would allow discovery of $t \rightarrow H^{+} b$ for $m_{t}-m_{H^{*}} \geq 25 \mathrm{GeV}$. 


\subsubsection{SUSY Searches in Gluino Pairs}

ISASUSY[10] (ISAJET Version 7.0) was used to compute the required luminosity to produce 10000 gluino pairs in $10^{7}$ seconds in $p p$ and $p \vec{p}$ collisions. The center-of-mass energy was varied between $2 \mathrm{TeV}$ and $80 \mathrm{TeV}$. The mass of the gluino was set to 300 $\mathrm{GeV}, 1000 \mathrm{GeV}$ and $3000 \mathrm{GeV}$. The minimal supersymmetric extension to the standard model was assumed. The mass of the squark, stops, sleptons and sneutrinos was set to twice the gluino mass, and $\tan (\beta)=5$ and $\mu=-300 \mathrm{GeV}$ were assumed. The mass of the supersymmetric Higgs was set to $500 \mathrm{GeV}$ and the top quark mass was fixed to $150 \mathrm{GeV}$. Cross sections were integrated between jet $p_{T}$ of $10 \mathrm{GeV}$ and $1 \mathrm{TeV}$ for the light and intermediate mass gluinos and $10 \mathrm{GeV}$ and $10 \mathrm{TeV}$ for the heavy gluinos.

From Fig. 6 we conclude that the SSC ( $40 \mathrm{TeV}$ center-of-mass energy and nominal luminosity of $\left.10^{33} \mathrm{~cm}^{-2} \mathrm{sec}^{-1}\right), 300 \mathrm{GeV}$ or $1 \mathrm{TeV}$ gluinos are within easy reach, while $3 \mathrm{TeV}$ gluinos would be much harder. However, such heavy gluinos would not solve the fine tuning problem which is one of the main motivations for SUSY. The upper limit of about $2 \mathrm{TeV}$ covers the expected range of SUSY masses related to electroweak symmetry breaking comfortably. 


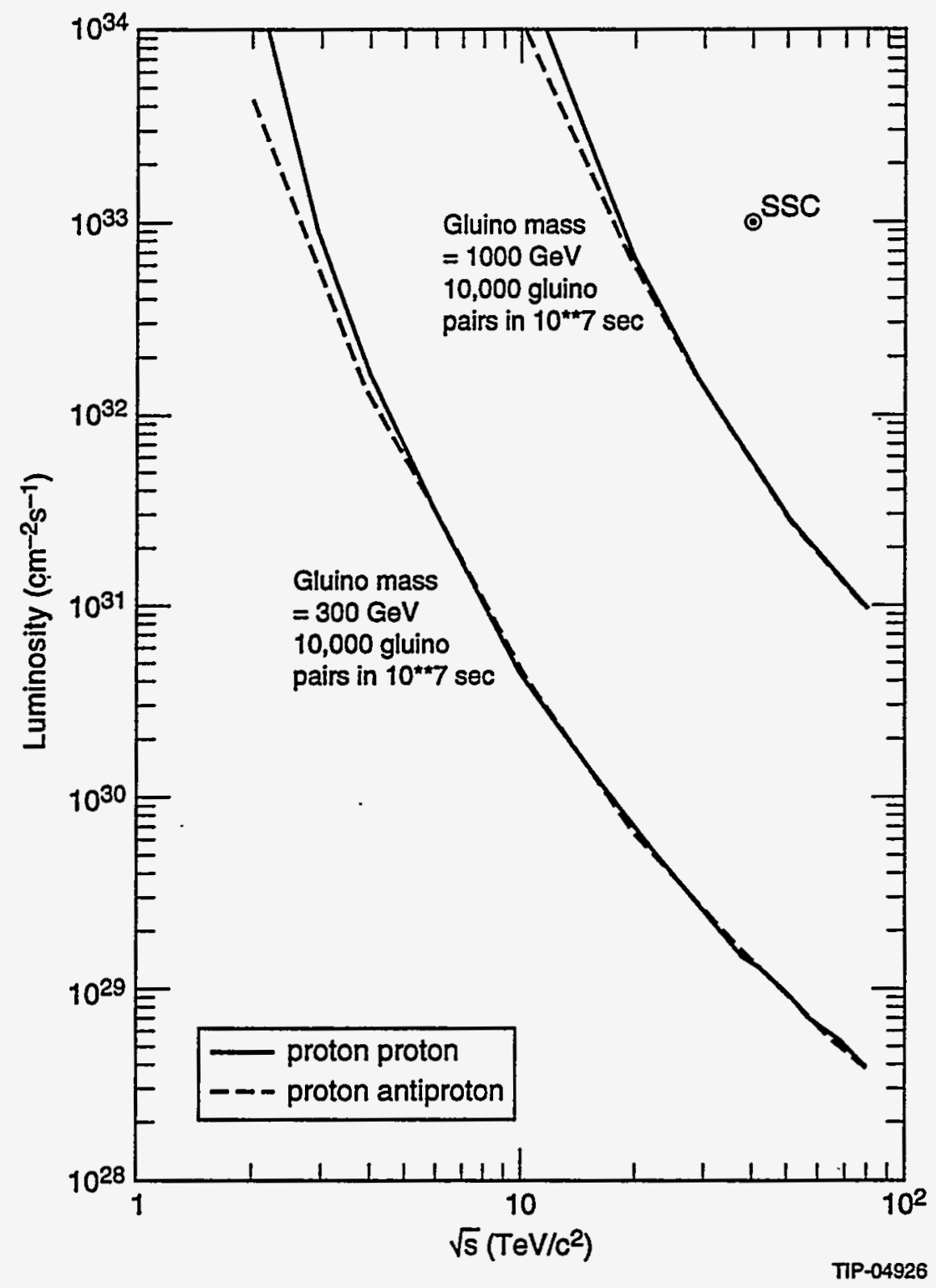
Fig. 6a. Instantaneous luminosity needed to produce 10000 gluino pairs vs. $\sqrt{s}$ for $\mathrm{M}_{\tilde{g}}=300 \mathrm{GeV}$ and $1000 \mathrm{GeV}$. This is approximately
the discovery limit. 


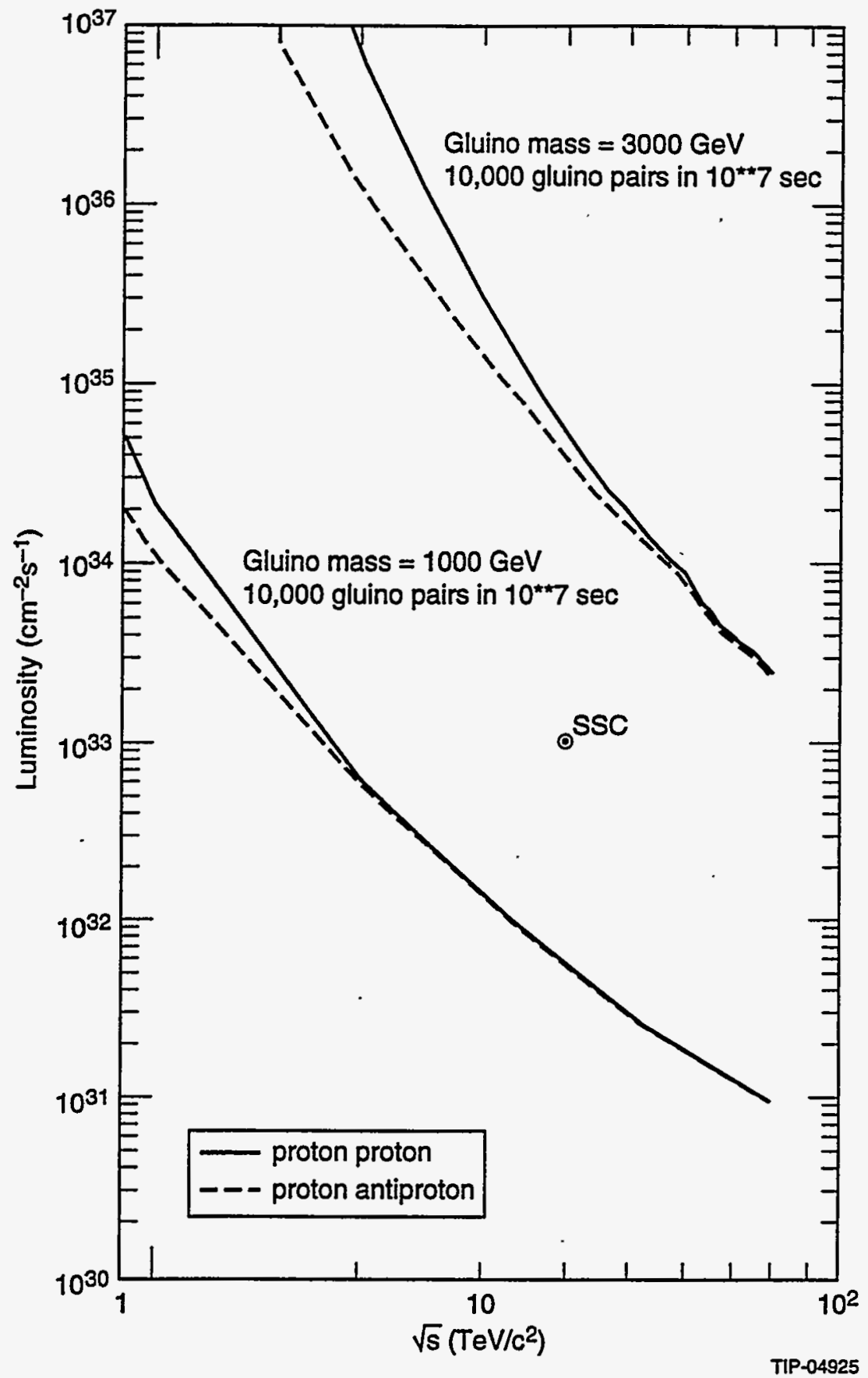
Fig. 6 b. Instantarieous luminosity needed to produce 10000 gluino pairs vs. $\sqrt{s}$ for $M_{\tilde{g}}=1000 \mathrm{GeV}$ and $3000 \mathrm{GeV}$. This is
approximately the discovery limit. 


\subsubsection{Heavy Gauge Vector Boson Searches}

The cross section for the production of additional $Z^{\prime}$ vector bosons has been investigated using the PYTHIA [9] generator. Only the $Z^{\prime}$ contribution to the propagator has been used. The width of the $Z$ is assumed to increase linearly with the mass, corresponding to the same couplings to quarks and leptons as in the standard model $Z^{0}$. Figure 7 shows the luminosity required to produce 100 events per SSC year for different masses of the $Z$ as a function of the center-of-mass energy for the $p p$ and $p \bar{p}$ case. The systematic errors $(\sim 40 \%)$ on the cross section values are mainly due to the structure functions. 


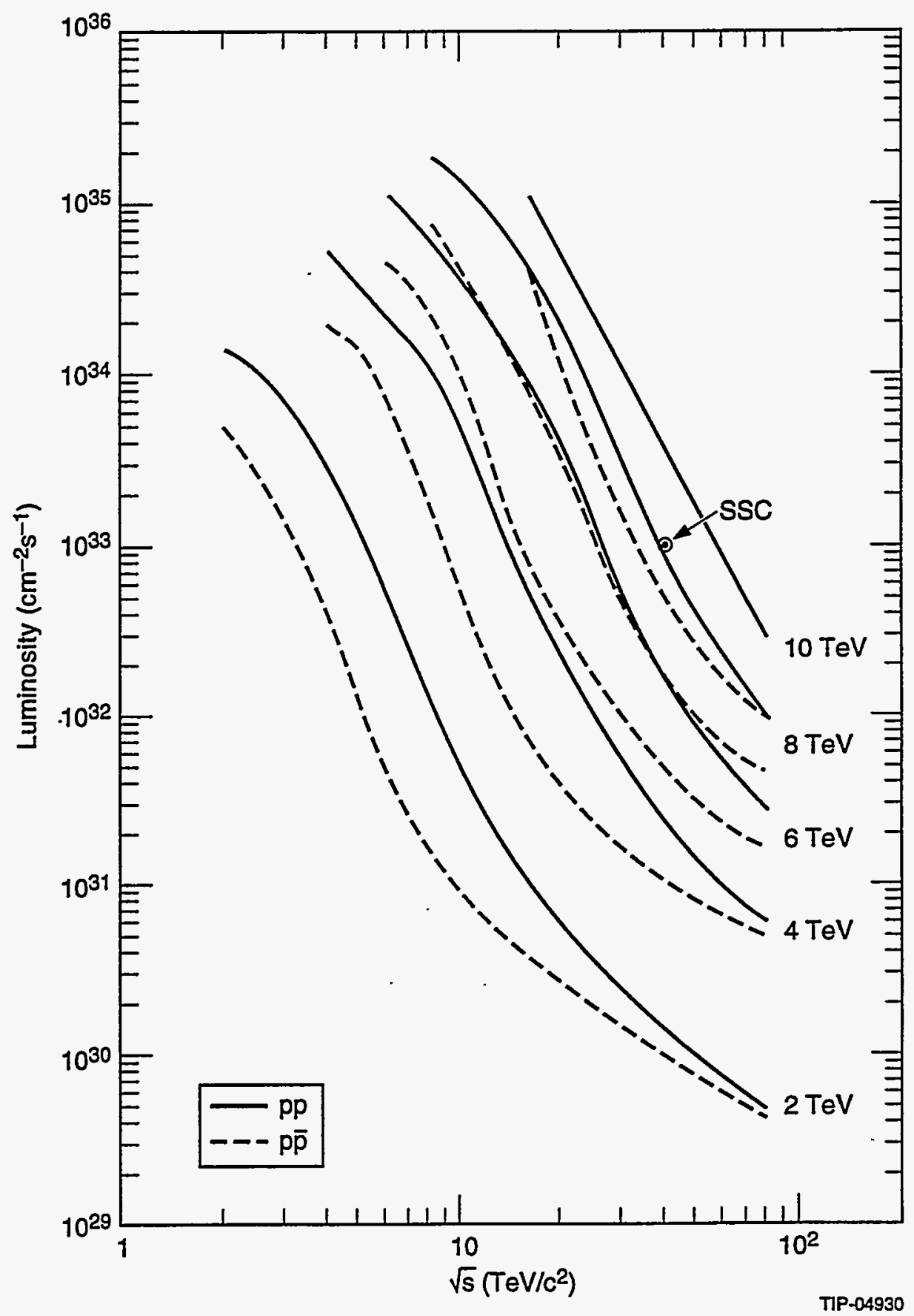

Fig. 7. The curves indicate the luminosity needed to produce $100 Z$ events/year in $p p$ (solid) $p \bar{p}$ (dotted) interactions. No branching ratios are included. 
The difference between the $p p$ and $p \bar{p}$ initiated cross sections can be explained in terms of the valence quark contributions for the $\bar{p}$ 's. The difference is about a factor of 5 for the heaviest observable $Z^{\prime}$.

\subsubsection{Excited Quark Searches}

The discovery limits have been studied for excited quark decays into $Z^{0}+$ jets using the model of Baur and Zerwas [11] in the PYTHIA 5.6 generator [9]. In this study we assume the following parameters: $f_{s}=f=f^{\prime}=1, m_{u^{*}}=m_{d^{*}}=\Lambda_{c}$ The limit was determined for $10^{7}$ seconds running and

$$
\frac{\mathrm{N}_{\text {signal }}}{\sqrt{\mathrm{N}_{\text {background }}}} \geq 5.0 \text {. }
$$

$Z^{0}+$ quark (or gluon) events were generated as the background. The event selection required at least two muons in the range $70 \mathrm{GeV} \leq M_{\mu \mu} \leq 100 \mathrm{GeV}$, with $p_{t \mu l} \geq 50 \mathrm{GeV} / \mathrm{c}$ and $p_{t \mu 2} \geq 20 \mathrm{GeV} / \mathrm{c}$, in addition to one jet $\left(E_{t} \geq 100 \mathrm{GeV}\right.$; with $\left.R=0.8\right)$ in the opposite hemisphere of the muons. The signal was determined in a window representing four times the decay width, about the central value. Figure 8 shows the luminosity required for the different masses.

We note, however, that searches involving the contribution from the contact interactions ${ }^{1}$ are more sensitive than the direct searches. If excited quarks exist, there would also be new interactions between quarks with a scale $\Lambda \sim M$. At $\sqrt{s}=40 \mathrm{TeV}$ and $L=10^{33} \mathrm{~cm}^{-}$ ${ }^{2} \mathrm{~s}^{-1}$, one is sensitive [5], [7] to $\Lambda \geq 25 \mathrm{TeV}$. 


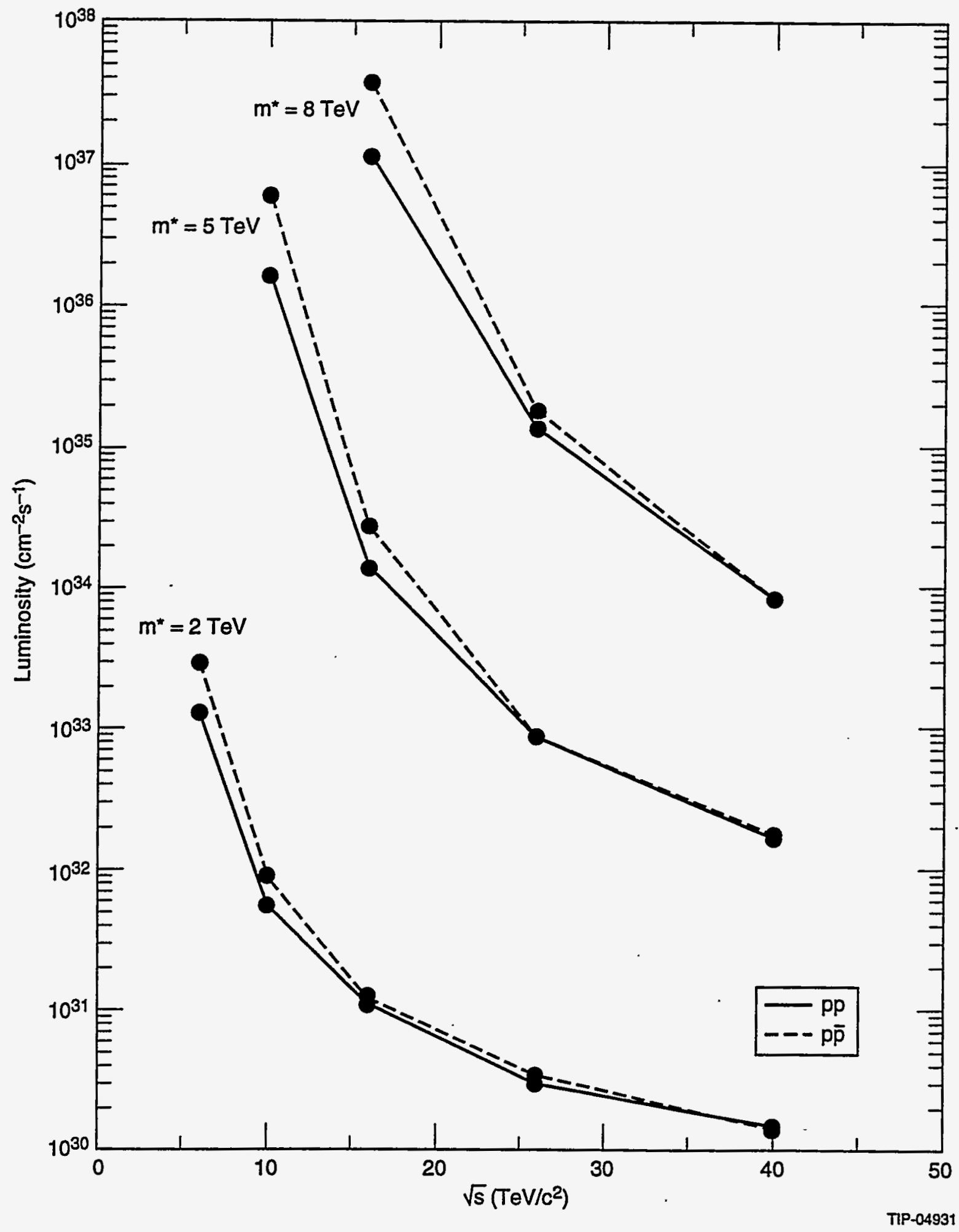

Fig. 8. Luminosity required to observe a $5 \sigma$ effect from $q^{*} \rightarrow Z+q$ in the model of ref. 6 . See Section 5 for discussion. 


\subsection{Accelerator Limitations}

Luminosity Limits:

Upper limits on the SSC luminosity as a function of energy can be readily generated, based on a few assumptions:

1. The ultimate luminosity must not violate the head-on beam-beam tune shift. This tune shift has been historically set at less than 0.005 . Observations from the CERN $\bar{p}$ p collider show serious beam lifetime degradation at values above 0.004 . We shall use this value as a limit.

2. The amount of synchrotron radiation allowable is limited by the ability of the cryogenic system to remove the induced heat. This depends on the capacity of the installed system. For the present Collider design, this amounts to $11.25 \mathrm{~kW} /$ ring of capacity to remove heat due to synchrotron radiation. This capacity can easily be increased, but with a high price [12].

3. The emittance of the beams is only variable over a small range. The design value is $\mathrm{E}_{\mathrm{N}}=1 \pi$ $\mathrm{mm}$-mrad. This can be increased up to a value of $4 \pi \mathrm{mm}$-mrad, at which point the physical aperture of the vacuum pipe will limit the increase. Further increase would be very expensive.

4. The bunch spacing is set at one bunch per five meters. Every bunch is assumed filled.

5. The present Collider interaction region design with an interaction point value of $\beta^{\star}=0.5 \mathrm{~m}$ is assumed.

Using the above assumptions, the maximum achievable luminosity at a given energy can be calculated. For energy scaling, 4 to $40 \mathrm{TeV}$ in the Center of Mass is assumed possible in the Collider, although the lower-energy lifetime may be a problem. Energies below $4 \mathrm{TeV}$ would require collisions in the HEB. An HEB interaction geometry capable of developing a collision point in the HEB. An HEB interaction geometry capable of developing a collision point $\beta^{*}=$ $0.5 \mathrm{~m}$ is assumed.

Using the above assumptions, the maximum achievable luminosity at a given energy can be calculated. For energy scaling, 4 to $40 \mathrm{TeV}$ in the Center of Mass is assumed possible in the Collider, although the lower-energy lifetime may be a problem. Energies below $4 \mathrm{TeV}$ would require collisions in the HEB. An HEB interaction geometry capable of developing a collision $\beta^{\star}=1 \mathrm{~m}$ has been assumed.

The head-on beam-beam tune shift is given by:

with:

$$
\Delta v=\mathrm{N}_{\mathrm{Br}} / 4 \pi \mathrm{E}_{\mathrm{N}} \leq 0.004
$$

$$
\begin{aligned}
& N_{B}=\text { Number of particles per bunch } \\
& E_{N}=\text { Normalized emittance } \\
& \mathfrak{r}_{p}=\text { classical proton radius }
\end{aligned}
$$


With this value for $N_{B}$, the luminosity is given by:

$$
L=N^{2}{ }_{B} c / 4 \pi \sigma^{2} S_{B}
$$

with:

$$
\sigma^{2}=E_{N} \beta / \beta \gamma=\text { beam size }
$$

and:

$$
\mathrm{S}_{\mathrm{B}}=\text { bunch spacing. }
$$

The results of these calculations are shown in Figure 2.3.1.

\subsection{References}

[1] "An Assessment of the Antiproton-Proton Option for the SSC," prepared by the pbarp study group of the Central Design Group, B. Barish, chair, in 1986, SSC-SR-1022

[2] E. Eichten, I. Hinchliffe, K. Lane, and C. Quigg, Rev. Mod. Phys. 56, 579 (1984)

[3] S. D. Drell, et al., DOE/ER-0434 (Jan., 1990)

[4] J. Boucrot, et al, "Search for Neutral Higgs at LEP 200," ECFA Workshop on LEP 200, CERN Report 87-08, p. 312

[5] GEM Collaboration, GEM Technical Design Report, GEM-TN-93-262

[6] K. Einsweiler, SDC Note SDC-91-00095

[7] SDC Collaboration, SDC Technical Design Report, SDC-92-201

[8] S. Mrenna, et al, GEM-TN-93-373

[9] T. Sjostrand, CERN-TH.6488/92

[10] H. Baer, F. Paige, S. Protopopescu, and X. Tata, SSCL-Preprint-441 (1993)

[11] U. Baur and P.M. Zerwas, Phys. Rev. D42 (1990) 815

[12] As an example, in order to increase the luminosity at $40 \mathrm{TeV}$ from the current $1033 \mathrm{~cm}-2 \mathrm{~s}-1$ by an order of magnitude, the refrigeration would need to be doubled. This would mean acquiring ten additional cryo plants and fully developing the 'zero' sites to equal the 'five' sites. The approximate cost for this upgrade is $200 \$ \mathrm{M}$. 


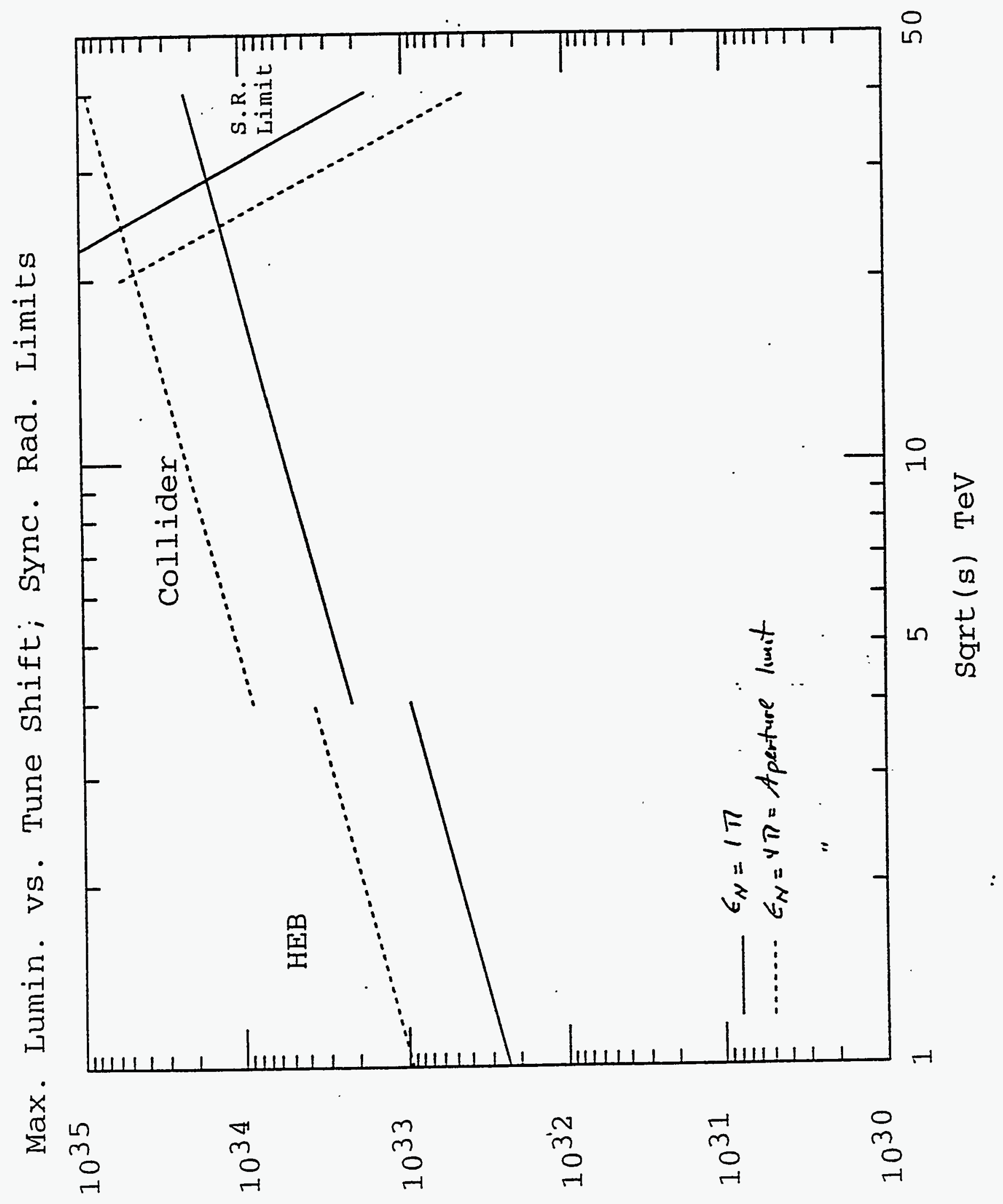

s/ $2 * *$ UT⿰/ K7TุSOUṬunT 


\section{Proton-Antiproton Collider at $20 \times 20 \mathrm{TeV}$}

\subsection{Program Summary}

In this option, a single $20 \mathrm{TeV}$ ring is constructed, with an antiproton source, so that $40 \mathrm{TeV}$ collisions take place between proton and antiproton beams counter-rotating in the same beam pipe. There is a potential for cost reduction by eliminating one of the two collider rings and the optics for the cross-over of the two beams at the interaction regions, though an antiproton source must be added. Many questions are thus opened for discussion so that the ground rules for this option strongly affect any estimate of cost or performance. There are two extremes: make the minimum machine changes to obtain $p \bar{p}$ collisions at $40 \mathrm{TeV}$ at whatever luminosity is thus obtained, or to try to obtain a luminosity which allows most of the physics program to be followed.

In the first case, the luminosity will likely be less than $10^{32} \mathrm{~cm}^{-2} \mathrm{~s}^{-1}$; in the latter case, many machine changes must be made, for example, the magnet apertures are likely to be increased substantially [1]. It should be remembered that the largest increase in estimated cost for the SSC was due the increase of magnet aperture from $40 \mathrm{~mm}$ to $50 \mathrm{~mm}$. In addition, the detectors may have to be upgraded to handle larger numbers of interactions per bunch crossing. Detailed study is necessary before cost reductions can be guaranteed.

The major physics issue is that an estimate of the luminosity of a $p \bar{p}$ collider, using safe extrapolation of existing technology, is about $10^{32} \mathrm{~cm}^{-2} \mathrm{~s}^{-1}$; a factor of ten less than that of the baseline $p p$ design. Since the physics reach of a multi- TeV collider depends more on its luminosity and center-of-mass $(\mathrm{cm})$ energy than on whether protons or antiprotons are used, this option is not competitive with an LHC that would begin to operate in the same time frame.

The machine evolution is divided into two phases. In Phase 1 , one $20 \mathrm{TeV}$ ring is constructed, with an antiproton source; a luminosity of roughly $10^{32} \mathrm{~cm}^{-2} \mathrm{~s}^{-1}$ is attainable. In Phase 2 (which would be funded separately), the second collider ring and new interaction optics are added, and a switch is made to proton-proton collisions, to reach a luminosity in the range $10^{33} \mathrm{~cm}^{-2} \mathrm{~s}^{-1}$ to $10^{34} \mathrm{~cm}^{-2} \mathrm{~s}^{-1}$.

The construction of this option could involve some, or all, of the following changes to the baseline design:

- The antiproton source, with two or three additional rings, a target and injection line, and new operating modes for the MEB and HEB, must be designed, constructed, and brought into operation.

- An increase in the aperture of the collider magnets, perhaps to $60-70 \mathrm{~mm}$, to allow separation of the proton and antiproton beams.

- Increase in the MEB cycle rate (to $1 \mathrm{~Hz}$ from the present $0.12 \mathrm{~Hz}$ ).

- An increase in LINAC energy (from $600 \mathrm{MeV}$ to $1 \mathrm{GeV}$ ), to improve proton beam intensity.

- Cooling of the protons as well as of the antiprotons to achieve low emittance and high intensity.

- An increase in the average number of interactions per bunch crossing, which would have a cost impact on detectors. 
- Maintenance of $\mathrm{HEB}$ energy at $2 \mathrm{TeV}$ (or even an increase).

- The antiproton source has in addition, several options:

- Debuncher and accumulator of LEB circumference to increase number of bunches

- Multiple targetting to overcome target heating limitation

The proton-antiproton option would have the following disadvantages compared with the $p p$ baseline:

- Lower luminosity in $p \bar{p}$ mode, limiting the initial physics program.

- Reduced availability of beam due to the complexity of operating the antiproton source.

- Recovery from a loss of stored beam would take much longer than for a $p p$ collider due to the need to make a new fill of antiprotons. The combination of this and the previous item is likely to reduce the availability of collisions by a factor of two, based on SppS experience [1]

- Moving from Phase 1 to Phase 2 would require a shutdown of at least two years and incur a large additional cost.

- Tighter tolerances on many collider components.

A proton-antiproton collider is more risky than its proton-proton counterpart, and it is incapable of providing the $10^{34} \mathrm{~cm}^{-2} \mathrm{~s}^{-1}$ luminosity eventually achievable in a proton-proton collider. While a proton-antiproton collider with an average luminosity of $10^{33} \mathrm{~cm}^{-2} \mathrm{~s}^{-1}$ has not been technically ruled out at present, it would require more than an order of magnitude improvement over existing antiproton source technology as well as an increase in the collider dipole aperature.

The goals of the physics program would be the same as that for the baseline design (though it would clearly be pursued in a less effective way), so that all work done on design and construction for GEM and SDC would be used. The change from Phase 1 (single ring) to Phase 2 (two rings) would necessitate a change in detector height, a change in magnet height, or a set of interaction region dipoles (the cost of this has not been estimated, but would be part of the Phase 2 cost).

Several studies have been made of this option. The most recent document is Ref. 1 . References to earlier work can be found in this document. Since the time of that study there have been a few changes:

- The cross section for antiproton production has been determined to be about a factor of two lower than assumed at the time of the study.

- The number of active interaction regions has declined, from six to two full-luminosity regions.

- Machine parameters have changed, including MEB and HEB circumference and energy and collider aperture.

- The favored mass of the top quark has increased to the region of $150 \mathrm{GeV}$.

Each of these changes modifies the conclusions of Ref. 1 in detail. 


\subsection{Physics Program}

\subsubsection{Overview}

The goals of the physics program would be the same as those of the baseline SSC design, namely the exploration of electroweak symmetry breaking and the search for physics beyond the Standard Model. (Additional options, not considered here, might include a low energy antiproton physics program.) As a consequence, the detector requirements are the same. In particular, the discovery and study of supersymmetry, possible at modest luminosity, requires hermetic calorimetry and good identification and measurement of leptons. The existing detector designs would be used, and may even need improvement to maintain physics capability, as noted below.

The physics potential of $p p$ and $\not{p}$ at the same energy and luminosity is nearly identical except for those processes with large enough couplings that large masses, $M \geq 0.1 \sqrt{s}$, can be reached. Otherwise, valence quarks do not play an important role, and the gluon and sea-quark distributions are identical for $p p$ and $\bar{p}$. In the SSC energy and luminosity range, the only such processes are expected to be the production of $Q C D$ jets and the production of new $W^{\prime}$ or $Z^{\prime}$ boson. The jet cross sections in $p p$ and $p \bar{p}$ are nearly equal. The cross sections for $W^{\prime}$ and $Z^{\prime}$ at the highest observable masses, $\mathrm{M} \sim 8 \mathrm{TeV}$, are about an order of magnitude larger for $p \bar{p}$ than for $p p$. Hence, if the luminosity for $p \bar{p}$ is an order of magnitude less than that for $p p$, then the physics performance for $p p$ is better for all processes except the heaviest $W^{\prime}$ and $Z^{\prime}$ bosons, for which it is comparable. This has been confirmed by detailed calculations of a wide variety of processes; see, e.g., the Workshop on $p \bar{p}$ Options for the Supercollider (Chicago, 1984) and Eichten, Hinchliffe, Lane, and Quigg (EHLQ)[2].

To make a comparison between the physics reach of various possibilities, we use several sources, including the work of EHLQ. Some arbitrary choices were made in EHLQ, so that limits based on this are not absolute, but should be comparable between $p p$ and $\bar{p} p$.

In Table 3.1 we list the 'physics reach' for various processes for some combinations machine parameters, for a 'year' of $10^{7}$ seconds. The coverage of a $\bar{p} p$ machine at a luminosity of $10^{32}$ is clearly less than that of a $p p$ machine at 1033 . It is clearly inadequate for a Higgs search, since the mass of the Higgs can lie anywhere from the current lower limit (about $60 \mathrm{GeV}$ ) to a theoretical upper limit of $650-800 \mathrm{GeV}$. Also, the range of coverage of the Higgs mass is incomplete even for $p p$ at $10^{33}$, since it will be necessary to go down to $80 \mathrm{GeV}$, and that will take either several years' running or higher luminosity.

Table 3.1: Physics reach (limits or lower limits) for one year ( $10^{7}$ seconds) of running at given effective luminosity (actual luminosity will depend on the detector).

\begin{tabular}{|l|l|l|l|l|}
\hline Particles: & \multicolumn{2}{|c|}{ Proton-proton @ 40 TeV } & \multicolumn{2}{c|}{ Proton-antiproton @ 40 TeV } \\
\hline Physics & $10^{32} \mathrm{~cm}^{-2} \mathrm{~s}^{-1}$ & $10^{33} \mathrm{~cm}^{-2} \mathrm{~s}^{-1}$ & $10^{32} \mathrm{~cm}^{-2} \mathrm{~s}^{-1}$ & $10^{33} \mathrm{~cm}^{-2} \mathrm{~s}^{-1}$ \\
\hline \hline $\begin{array}{l}\text { Precise mass of top } \\
\text { quark }\end{array}$ & $200 \mathrm{GeV}$ & $300 \mathrm{GeV}$ & same & same \\
\hline New heavy quark & not calculated & $1.7 \mathrm{TeV}$ & $1.2 \mathrm{TeV}$ & $1.8 \mathrm{TeV}$ \\
\hline
\end{tabular}




\begin{tabular}{|l|l|l|l|l|}
\hline $\begin{array}{l}\text { New heavy } \\
\text { (charged) lepton }\end{array}$ & $0.25 \mathrm{TeV}$ & $0.55 \mathrm{TeV}$ & $0.27 \mathrm{TeV}$ & $0.65 \mathrm{TeV}$ \\
\hline New boson $\left(\mathrm{W}^{ \pm}\right)$ & $4.2 \mathrm{TeV}$ & $7 \mathrm{TeV}$ & $4.8 \mathrm{TeV}$ & $8 \mathrm{TeV}$ \\
\hline New boson $\left(\mathrm{Z}^{\circ}\right)$ & $3.5 \mathrm{TeV}$ & $5.5 \mathrm{TeV}$ & $4 \mathrm{TeV}$ & $7 \mathrm{TeV}$ \\
\hline $\begin{array}{l}l l \text { compositeness } \\
\text { scale }\end{array}$ & not calculated & $28 \mathrm{TeV}$ & $25 \mathrm{TeV}$ & $36 \mathrm{TeV}$ \\
\hline Higgs mass range & $200-300 \mathrm{GeV}$ & $95-800 \mathrm{GeV}$ & $200-300 \mathrm{GeV}$ & $95-800 \mathrm{GeV}$ \\
\hline Gluino/squark mass & $0.2-1 \mathrm{TeV}$ & $0.2-2 \mathrm{TeV}$ & $0.2-1 \mathrm{TeV}$ & $0.2-2 \mathrm{TeV}$ \\
\hline
\end{tabular}

From the physics point of view, the $10^{32} \bar{p} p$ option is unsatisfactory in that it leaves known and extensive gaps in its discovery potential. We would be building an instrument that we know would have blind spots.

\subsubsection{Detector Issues}

As noted above, the physics program that is possible with $\mathrm{pp}$ at $40 \mathrm{TeV}$ has much in common with the baseline program. In particular, the discovery and study of supersymmetry, possible at modest luminosity, requires hermetic calorimetry and good identification and measurement of leptons. The existing detector designs would be used, and may even need improvement.

One significant difference if high luminoisity were to be obtained by increasing the average number of interactions per bunch crossing, $n$, e.g. to $\mathrm{n}=37$ for $\mathrm{L}=2 \times 10^{32} \mathrm{~cm}^{-2} \mathrm{~s}^{-1}$. The GEM [3] and SDC [4] detectors have planned to accept up to $n=1.6$ for most of their physics program (at a luminosity of $10^{33} \mathrm{~cm}^{-2} \mathrm{~s}^{-1}$ ). They also have studied detector performance at up to $n=16$ for work at a luminosity of $10^{34} \mathrm{~cm}^{-2} \mathrm{~s}^{-1}$, where they may turn off or ignore central subdetectors due to high occupancy or radiation damage.

The LHC detectors have accepted $n=40$ as a baseline scenario, but by using apparatus which has compensating features at higher cost, and by exploiting the luminosity of $10^{34} \mathrm{~cm}^{-2} \mathrm{~s}^{-1}$ to obtain a sensitivity about equal to that of the SSC baseline for some cases.

The case of high $n$ but modest luminosity requires study. The silicon vertex trackers would continue to operate, but pattern recognition of 40 vertices would be hard; a pixel vertex detector might be needed. In the case of SDC, the straw-tube outer tracker has an occupancy of $10 \%$ in its first superlayer at $n=1.6$ (see Table 4-12 of [3]); at $n=40$, this will exceed $100 \%$, making this tracker layer useless. Even the outer superlayer occupancy would exceed $50 \%$. So an improved tracker would be required. In the case of GEM, the search for an intermediate mass Higgs would require improved resolution, segmentation, and pointing in the EM calorimeter to get useful sensitivity. Sensitivity would be reduced by the increased noise and pileup. In both cases, some modification in the electronics may be needed to handle the greater amount of data per event. No study has been done to establish the need for, or to estimate the cost of, these improvements, but an increase in cost of $\$ 100 \mathrm{M}$ per detector seems not unreasonable.

\subsection{Relationship to Other Programs}

Other relevant programs include LEP200, the FNAL Tevatron, and the LHC. LEP200 will be able to find a Higgs up to about $80 \mathrm{GeV}$, defining the lower limit for SSC searches. The 
Tevatron, with the main injector, will be able to look for top quarks up to about $170 \mathrm{GeV}$ and compositeness to a scale of 5 or so $\mathrm{TeV}$.

LHC is a proton-proton collider designed to be directly competitive with the SSC, using a cm energy of $14 \mathrm{TeV}$ and a luminosity of $2 \times 10^{34} \mathrm{~cm}^{-2} \mathrm{~s}^{-1}$. It is not yet approved, but current plans indicate a startup date around 2004 . The high luminosity is designed to compensate for the lower energy, but produces experimental difficulties, which, however, the LHC detector collaborations believe they can overcome. A $p \bar{p}$ collider at a luminosity of $10^{32} \mathrm{~cm}^{-2} \mathrm{~s}^{-1}$ would not be competitive with LHC unless it were to begin operating earlier. Thus the main questions are what the schedule of a $p \bar{p}$ collider could be and what luminosity can be attained.

\subsection{Machine Configuration}

Here we discuss the machine configuration for a luminosity of about $10^{32} \mathrm{~cm}^{-2} \mathrm{~s}^{-1}$, followed by a discussion of the possibility of attaining luminosities as high as $10^{33} \mathrm{~cm}^{-2} \mathrm{~s}^{-1}$. For this, there are two main issues to consider: how fast can antiprotons be produced and can machine parameters be adjusted so that antiprotons can be used up fast enough to allow the desired luminosity? These issues will now be discussed briefly.

\subsubsection{The Antiproton Source}

Here we describe a minimum-cost approach. Antiproton production would be carried out in a series of steps, initially following the Fermilab and CERN models [5] and as described in Ref. 1:

1. Antiprotons would be produced by accelerating protons through the linac, LEB and MEB and extracted in one turn onto a target at 120 - $200 \mathrm{GeV}$. The MEB would run with a threesecond cycle time. The thermal shock in the target limits the beam intensity.

2. Antiprotons produced at about $10-12 \mathrm{GeV}$ would be focused by a lithium lens and sent on an injection beam line to a debuncher/accumulator ring.

3. In the LEB-size debuncher, the bunches would be manipulated in longitudinal phase space and stochastically precooled.

4. The antiprotons are then sent to an accumulator and stochastically cooled further and accumulated in a 'stack.'

The target, injection line, debuncher and accumulator would be in a new complex.

5. Once a day, the collider is prefilled with protons to match the $\bar{p}$ bunches.

6. Once a day, the antiproton stack is transferred to the MEB in two 100-bunch batches and thence to the collider.

Therefore, a proton-antiproton collider will require two additional rings (debuncher and accumulator), additional technologies (stochastic cooling and antiproton formation), new operating modes and machine upgrades beyond what is contemplated for the present SSC design.

The production capability of this scenario for the SSC can be estimated by extrapolation from the FNAL experience Ref. 5 as shown in Table 3.2 (column SSC 1). The major 
Table 3.2: Estimate of antiproton accumulation rate for the SSC

\begin{tabular}{|l|l|l|l|l|}
\hline \multicolumn{2}{|l|}{ Parameter } & FNAL & SSC 1 & SSC 2 \\
\hline 1. & Beam momentum & $120 \mathrm{GeV} / \mathrm{c}$ & $180 \mathrm{GeV} / \mathrm{c}$ & $180 \mathrm{GeV} / \mathrm{c}$ \\
\hline 2. & Relative production cross section & 1.0 & 1.5 & 1.5 \\
\hline 3. & Cycle time & $2.4 \mathrm{~s}$ & $3.0 \mathrm{~s}$ & $1.0 \mathrm{~s}$ \\
\hline 4. & No. of protons per cycle & $1.8 \times 10^{12}$ & $6 \times 10^{12}$ & $1.2 \times 10^{13}$ \\
\hline 5. & Beam spot size & $0.15 \mathrm{~mm}$ & $0.5 \mathrm{~mm}$ & $1.0 \mathrm{~mm}$ \\
\hline 6. & Target length (Cu) & $70 \mathrm{~mm}$ & $70 \mathrm{~mm}$ & $70 \mathrm{~mm}$ \\
\hline 7. & Debuncher acceptance & $28 \pi \mathrm{mm}$-mrad & $40 \pi \mathrm{mm}-\mathrm{mrad}$ & $40 \pi \mathrm{mm}$-mrad \\
\hline 8. & Nominal fraction within acceptance & 0.34 & 0.36 & 0.21 \\
\hline 9. & Production momentum & $8.9 \mathrm{GeV} / \mathrm{c}$ & $12 \mathrm{GeV} / \mathrm{c}$ & $12 \mathrm{GeV} / \mathrm{c}$ \\
\hline 10. & $\Delta \mathrm{p} / \mathrm{p}$ & 0.04 & 0.06 & 0.06 \\
\hline 11. & $\begin{array}{l}\text { Ratio SSCL/FNAL [parameters } \\
2,3,4,8,9,10]\end{array}$ & 1.0 & 8.6 & 30. \\
\hline 12. & Accumulation rate & $4 \times 10^{10} \mathrm{pbar} / \mathrm{hr}$ & $\begin{array}{l}0.33 \times 10^{12} \mathrm{pbar} / \mathrm{hr} \\
\left(1 \times 10^{8} \mathrm{pbar} / \mathrm{s}\right)\end{array}$ & $\begin{array}{l}1.2 \times 10^{12} \mathrm{pbar} / \mathrm{hr} \\
\left(3.0 \times 10^{8} \mathrm{pbar} / \mathrm{s}\right)\end{array}$ \\
\hline 13. & Transfer efficiency to collider & -- & 0.75 & 0.75 \\
\hline 14. & $\begin{array}{l}\text { No. of antiprotons in collider }(22 \mathrm{hr} \\
\text { stack) }\end{array}$ & -- & $0.6 \times 10^{13}$ & $1.7 \times 10^{13}$ \\
\hline
\end{tabular}

factors which would change the accumulation rate are shown; other factors are assumed to be the same. The observed performance of the FNAL source is used. Target heating is controlled by using a larger beam so that more protons per spill can be used; column 'SSC 2' is based on the same information, and uses a fast-cycling MEB and an MEB-sized antiproton storage ring, and shows that acceptance begins to limit the usefulness of this approach (twice the beam gives only $17 \%$ more antiprotons).

Multiple targets and/or multiple extractions from the MEB might be able to overcome the heating in the target and make use of the $4 \times 10^{13}$ capability of the $\mathrm{MEB}$, to give a factor of three increase in antiproton accumulation rate.

\subsubsection{Luminosity}

The luminosity obtained from the antiprotons depends on several factors, the most important being the emittance achieved and the number of bunches that are filled. In this section we use the parameters and default values given in Table 3.3

With these definitions, the luminosity is given by:

$$
\mathrm{L}=\frac{\beta \gamma f B \mathrm{~N}^{+} \mathrm{BN}^{-} B}{4 \pi \varepsilon_{N} \beta^{*}}
$$


A major limitation is the head-on tune shift, which is given for one beam by:

$\Delta v_{\mathrm{H} 0}=\frac{N_{B} r_{p}}{4 \pi \varepsilon_{N}}$

where the quantities on the RHS refer to the opposing beam.

The luminosity can be rewritten in terms of the head-on tune shift and the total number of p-bars as:

$\mathrm{L}=\frac{\beta \gamma f N_{\mathrm{tOt}} \Delta v_{\mathrm{HO}}}{r_{p} \beta^{*}}$

The average number of interactions per crossing is given by

$n=\frac{L \sigma_{\text {inel }}}{f B}$

There are two possible scenarios for filling the Collider. One choice is to maximize the luminosity. This is accomplished by putting as many particles in as few buckets as possible. The particle density is limited by the head-on beam-beam tune shift and the number of buckets limited by the total $\bar{p}$ production rate. The tune shift limits the number of particles per bucket to $3 \times 10^{10}$. (The actual tune shift increases slightly during the cycle due to synchrotoron radiation damping.) An optimal cycle to maximize integrated luminosity collides beams for about 22 hours, with a refill time of 2 hours. With a $\bar{p}$ production rate of $1 \times 10^{8} \bar{p}^{\prime} \mathrm{s} / \mathrm{sec}$, and a $\bar{p}$ transfer efficiency of $75 \%$, a total of $6 \times 10^{12} \bar{p}$ 's can be put into the Collider. This leads to filling 200 buckets in two LEB-length batches. It should be noted that here the luminosity is limited only by the beam-beam tune shift and the total number of $\bar{p}^{\prime}$ s produced, as well as the machine $\beta^{*}$. In particular, it does not depend on the beam emittance, and thus methods to reduce the emittance will not be of use.

This filling scenario has an advantage over the method examined in the 1986 study: namely that the protons and $\bar{p}$ 's are contained in two sausages, each approximately $1 \mathrm{~km}$ long. Thus, the beams only pass each other in two regions, the IRs on either side of the machine. In order to reduce the long-range beam-beam tune shift, the beams need to be separated only in these two straight sections, and thus, no increase in the apertures in the arc regions is needed.

The present design of the SSC injectors is for a proton density of $8 \times 10^{9}$ protons in a $1 \pi \mathrm{mm}$ mrad normalized emittance. This could probably be pushed to $1 \times 10^{10}$ protons per bunch, but further increase is believed to be difficult without an accompaning emittance increase. Thus, the current obtainable luminosity would be $7 \times 10^{31} \mathrm{~cm}^{-2} \mathrm{~s}^{-1}$. This obtainable luminosity would have an interaction rate of $\langle n\rangle=12$, significantly higer than the SSC baseline design. This defines Case 1 in Table 3.4.

If the $p$ bunches can be increased to $3 \times 10^{10}$ in $1 \pi \mathrm{mm}$-mrad, then the tune shift limit discussed above would be reached for both beams, and a luminosity of $2 \times 10^{32} \mathrm{~cm}^{-2} \mathrm{~s}^{-1}$ could be achieved. There would be $\langle n\rangle=37$ interactions per bunch crossing, which would have a significant impact on the detectors. This defines Case 2 in Table 3.4. Achieving it would require 
more understanding and operational experience. A substantial upgrade of the warm machines, including an increase in the LINAC energy to $1 \mathrm{GeV}$, might also be required.

The other possible scenario is to limit the number of interactions per crossing. Setting this value to $\langle n\rangle=2$, similar to the $p p$ design, the same number of $p$ 's collected in 22 hours would need to be spread into 850 buckets. This would be matched by 850 proton buckets, all having $7 \times 10^{9}$ particles each. In this case, the initial luminosity would be limited to $5 \times 10^{31} \mathrm{~cm}^{-2} \mathrm{~s}^{-1}$. This defines Case 3 in Table 3.4. In this case, since neither beam is near the beam-beam tune shift limit, some method of reducing the beam emittance, such as electron cooling, would increase the luminosity, but at the same time it would increase the number of interactions per crossing. The only way to increase the luminosity without increasing $\langle n\rangle$ would be to increase the number of $p$ 's produced, and put them into some larger number of buckets. In addition to the reduced luminosity, this scenario has another major drawback - the beams will now pass each other in the arc regions and so the arc components may need to be increased in aperture.

Table 3.3: Parameters, symbol and default values.

\begin{tabular}{|l|l|l|}
\hline Parameter & Symbol & Default value with units \\
\hline \hline Luminosity & $\mathrm{L}$ & $\mathrm{cm}^{-2} \mathrm{~s}^{-1}$ \\
\hline Lorentz factor & $\beta \gamma$ & $2.132 \times 10^{4}(\mathrm{at} 20 \mathrm{TeV} / \mathrm{c})$ \\
\hline $\begin{array}{l}\text { SSC revolution frequency @ 20 } \\
\text { TeV/c }\end{array}$ & $f$ & $3441 \mathrm{~Hz}$ \\
\hline $\begin{array}{l}\text { Number of filled bunches (of } \\
\text { antiprotons) }\end{array}$ & $B$ & \\
\hline $\begin{array}{l}\text { Number of particles per bunch } \\
\text { (antiprotons) }\end{array}$ & $N^{-} B$ & \\
\hline Number of protons per bunch & $N^{+} B$ & $0.6 \times 10^{13}$ \\
\hline $\begin{array}{l}\text { Total number of circulating } \\
\text { antiprotons }\end{array}$ & $N_{\text {tot }}=B N^{-} B$ & $1 \times 10^{-6} \mathrm{~m}$ \\
\hline $\begin{array}{l}\text { Normalized emittance (one } \\
\text { standard deviation) }\end{array}$ & $\varepsilon_{N}$ & $0.5 \mathrm{~m}$ \\
\hline $\begin{array}{l}\text { Betatron function at interaction } \\
\text { point }\end{array}$ & $\beta^{*}$ & $<0.004$ \\
\hline Head-on tune shift & $\Delta v$ & $120 \mathrm{mb}\left(1.2 \times 10^{-29} \mathrm{~m}^{2}\right)$ \\
\hline $\begin{array}{l}\text { Inelastic cross section for } \mathrm{pp} \\
\text { scattering at 20 TeV }\end{array}$ & $\sigma_{\mathrm{inel}}$ & $1.535 \times 10^{-18} \mathrm{~m}$ \\
\hline Classical proton radius & $r_{p}$ & $87.12 \mathrm{~km}$ \\
\hline $\begin{array}{l}\text { Average number of interactions per } \\
\text { bunch crossing }\end{array}$ & $<n>$ & \\
\hline SSC circumference & & \\
\hline
\end{tabular}


The three cases are summarized in Table 3.4. The conclusion, based on Fermilab operational experience, is that a luminosity of $0.7-2 \times 10^{32} \mathrm{~cm}^{-2} \mathrm{~s}^{-1}$ can be obtained in the Collider and is limited only by $\bar{p}$ production. The rate assumed here is an order of magnitude increase from the past, but is not an unobtainable goal. Any further increase in luminosity requires a proportionately greater $\bar{p}$ production rate, as well as a necessary increase in the aperture of the entire Collider. The more natural increase path would be to add another Collider ring and convert to $p p$ collisions. 
Table 3.4: Luminosity under various scenarios (values defining the scenario are in bold).

\begin{tabular}{|l|l|l|l|l|}
\hline Parameter & \multicolumn{1}{|c|}{$\mathrm{pp}$} & \multicolumn{3}{c|}{$\mathrm{p} \overline{\mathrm{p}}$} \\
\hline Case & Baseline & 1 & 2 & 3 \\
\hline \hline $\begin{array}{l}\text { Initial or } \\
\text { nominal } \\
\text { luminosity } \\
\left(\mathrm{cm}^{-2} \mathrm{~s}^{-1}\right)\end{array}$ & $\mathbf{1 0 ^ { 3 3 }}$ & $7 \times 10^{31}$ & $2 \times 10^{32}$ & $5 \times 10^{31}$ \\
\hline$n$ & 2 & 12 & 37 & \\
\hline$\Delta v(\mathrm{p})$ & 0.0009 & 0.0013 & 0.004 & 0.0009 \\
\hline$\Delta v(\overline{\mathrm{p}})$ & - & 0.004 & 0.004 & 0.0009 \\
\hline$B$ & $1.57 \times \mathbf{1 0}^{4}$ & $\mathbf{2 0 0}$ & $\mathbf{2 0 0}$ & 850 \\
\hline$N^{-}{ }_{B}$ & - & $3 \times 10^{10}$ & $3 \times 10^{10}$ & $7 \times 10^{9}$ \\
\hline$N^{+} B$ & $0.74 \times 10^{10}$ & $\mathbf{1 \times 1 0 ^ { 1 0 }}$ & $\mathbf{3 \times 1 0 ^ { 1 0 }}$ & $7 \times 10^{9}$ \\
\hline$N_{\text {tot }}$ & $1.2 \times 10^{14}$ & $\mathbf{6 \times 1 0 ^ { 1 2 }}$ & $\mathbf{6 \times 1 0 ^ { 1 2 }}$ & $\mathbf{6 \times 1 0 ^ { 1 2 }}$ \\
\hline$\varepsilon_{\mathrm{N}}(\mathrm{m})$ & $\mathbf{1 . 0 \times 1 0 ^ { - 6 }}$ & $\mathbf{1 . 0 \times 1 0 ^ { - 6 }}$ & $\mathbf{1 . 0 \times 1 0 ^ { - 6 }}$ & $\mathbf{1 . 0 \times 1 0 ^ { - 6 }}$ \\
\hline \hline $\begin{array}{l}\text { Beam separation } \\
\text { Location }\end{array}$ & - & $-10 \mathrm{~mm}$ & $\sim 10 \mathrm{~mm}$ & $\begin{array}{l}10-15 \mathrm{~mm} \\
\text { Entire ring }\end{array}$ \\
\hline
\end{tabular}

\subsubsection{Collider Issues}

There are several issues that need to be addressed by a detailed study.

The magnet aperture must allow separation of the two beams, enough to reduce long-range beam-beam interactions to a tolerable level. The minimum scenario outlined above avoids the issue of magnet aperture by having the beams pass each other in the straight sections.

In other scenarios, beam may pass in the collider dipoles. The effect on one beam scales as the total number of particles in the opposing beam, and depends on separation of the beams and emittance. Ref. 1 indicated a need for a beam separation of $15 \mathrm{~mm}$, leading to a dipole diameter of $61 \mathrm{~mm}$ (the then-current baseline had a diameter of $40 \mathrm{~mm}$ and an injection energy of $1 \mathrm{TeV}$ ); scaling from this gives beam separations of 7-10 $\mathrm{mm}$ for the values of Table 3.3, which might be accomodated in a $50 \mathrm{~mm}$ dipole. This requires detailed study. Control of beam-beam effects is easier in a distributed lattice [1] than in a clustered lattice like the present baseline. A change in the lattice would result in a mismatch to the baseline footprint, and possible land acquisition and tunneling costs.

The evolution of emittance as a function of time during a store affects the average luminosity and requires detailed study.

The interaction region is not simple; the beams must be kept separated as in the $p p$ option (depending on bunch spacing), and there will be a long range tune shift as complicated as in $p p$. It seems certain that there will be significant costs and risks associated with these issues, as well as schedule delays.

\subsubsection{Beam Separation}

The $\bar{F}$ and proton beams must be separated in the region of their passing in order to avoid an unacceptably large long-range tune shift. In the case with 200 bunches in each beam, this 
separation can be accomplished entirely within the long-straight sections on either side of the Collider. The most demanding separation will occur at injection energies, where the beam size is the maximum and so the aperture requirement will be the largest. During this time the beams will not be in collision. The long-range forces can be summarized by:

$\Delta v_{L_{R}}=\frac{2 \Delta v_{\text {HO }}}{(d / \sigma)^{2}}$

and

$\delta v=\frac{ \pm 12 \Delta v_{\mathrm{HO}}}{(d / \sigma)^{4}}$

where $\Delta v_{\mathrm{HO}}$ is the head-on beam-beam tune shift $\Delta v_{\mathrm{LR}_{\mathrm{R}}}, d$ is the beam separation, and $\sigma$ is the beam sigma. These quantities are per crossing. The \pm sign in the tune spread equation refer to the two planes.

For the above case, the total number of crossings per turn is 400 , and the tune shift and tune spread are:

$\Delta \mathrm{v}_{\mathrm{L}_{\mathrm{R}}}=0.0045$

$\delta v= \pm 0.00004$

for a beam-beam separation of $10 \mathrm{~mm}$. Detailed calculations and simulations will be required to determine whether this separation is possible within the present $50 \mathrm{~mm}$ aperture or whether the separation can be reduced somewhat to fit the magnets.

\subsection{Conclusion}

At the present time, a safe extrapolation from FNAL and CERN experience would yield an average luminosity of no more than 0.7 to $2 \times 10^{32} \mathrm{~cm}^{-2} \mathrm{~s}^{-1}$, possibly with a high number of interactions per bunch crossing. A proton-antiproton collider achieving a luminosity of $10^{33} \mathrm{~cm}^{-}$ $2 \mathrm{~s}^{-1}$ at one interaction point is not excluded but would require extensive $R \& D$, more than an order of magnitude increase in antiproton production rate above existing sources, rethinking of all machines, and a possible increase in the collider magnet apertures. Detector designs may have to be changed.

Otherwise, the impact on the physics program would be to reduce the discovery potential of the SSC program: necessary sensitivity would be reduced, or detector running time must be extended to ten or more years, straining the reliability of the apparatus or driving up its cost. As can be seen below, the assumptions made in a cost estimate can have very different results, ranging from almost no saving to very substantial.

\subsection{Cost Estimate}

A very rough, order-of-magnitude cost estimate for the above scenarios is given in Table 3.5 below. The cost savings from the elimination of one collider ring are based on the BCE. They do not take into account possible changes in unit cost from building only one ring. The cost of the $\bar{p}$ source is roughly estimated by scaling from the existing designs of the warm machines. Clearly the cost estimate is only a rough guess. 
Table 3.5: Rough cost estimate of the $\bar{p}$ option.

\begin{tabular}{|l|l|l|}
\hline Savings from eliminating one ring & & \\
\hline Deletion of one ring & $(1000)-(1300)$ & \\
\hline Contingency $(14 \%)$ & $(140)-(180)$ & \\
\hline Subtotal & & $(\mathbf{1 1 4 0}-(\mathbf{1 4 8 0})$ \\
\hline & & \\
\hline Baseline upgrades & & - \\
\hline MEB to 3 sec cycle & 6 & - \\
\hline Beam separation & 1 & - \\
\hline Other & 3 & 0 \\
\hline Increase IR aperture & 20 & \\
\hline $\bar{p}$ source & & \\
\hline Target \& transfer & 20 & \\
\hline Debuncher (scaled from LEB) & 50 & \\
\hline Accumulator (scaled from LEB) & 100 & \\
\hline Stoc. Cooling & 50 & \\
\hline Fast cycling MEB upgrade & 20 & \\
\hline Contingency (30\%) & 70 & \\
\hline Subtotal & & $(\mathbf{8 3 0}-(\mathbf{1 1 7 0 )}$ \\
\hline Total & & \\
\hline & & \\
\hline Possible additions & & \\
\hline Detector upgrade for large $<n>$ & 200 & \\
\hline Increased arc aperture if needed & 450 & \\
\hline $\bar{p}$ storage ring for increased $\bar{p}$ production & 250 & \\
\hline Linac upgrade for increased $\bar{p}$ production & 5 & \\
\hline MEB upgradefor increased $\bar{p}$ production & 100 & \\
\hline Contingency (30\%) & 120 & \\
\hline Subtotal & & \\
\hline
\end{tabular}

\subsubsection{References}

[1] "An Assessment of the Antiproton-Proton Option for the SSC," prepared by the pbar-p study group of the Central Design Group, B. Barish, chair, in 1986, SSC-SR1022

[2] E. Eichten, I. Hinchclifee, K. Lane, C. Quigg, Rev. Mod. Phys. 56, 579, 1984

[3] GEM Technical Design Report, GEM TN-93-262, SSCL-SR-1219, April 1993

[4] SDC Technical Design Report, SDC-92-201, SSCL-SR-1215, April 1992

[5] M.D. Church and J.P. Marriner, Ann. Rev. Nucl. and Particle Science (1993), to be published

\section{Missing Coil or Missing Magnet Schemes for $p-p$ collider at $10 \times 10 \mathrm{TeV}$}

\subsection{Program Summary (Missing Coils)}


The possibility of reducing the number of coils in a magnet ( 2 layer to 1 layer) and the resulting cost saving is estimated. The peak beam energy will be reduced to about $15 \mathrm{TeV}$. Designs to obtain higher fields in the magnets will be risky and it is not evident that such designs exist. The cost is based on using the present superconducting cable. The concept would be not be upgradable without changeout of magnets. The upgrading will involve disassembling all beam line equipments and may be categorized as installation of irradiated components, which can increase reinstallation cost.

\subsection{Physics Program}

Although at $\mathrm{CM}$ energies lower than $40 \mathrm{TeV}$, the Higgs production rate is lower by a factor depending on the Higgs mass, it appears that a beam energy of $15 \mathrm{TeV}$ has a finite possibility of finding a large mass Higgs Boson. However, a lower beam energy would result in a proportionately smaller luminosity that might have to be compensated by longer running times. Ultimately the luminosity might have to be raised above $10^{33}$ to obtain the same physics reach as at $40 \mathrm{TeV}$ if the Higgs remained elusive. The Collider could also be a good B factory or a Top factory at this energy. There are potential risks in field quality since 1 layer coils will have larger high order multipoles, which can increase cost for correctors or reduce beam quality (emittance) or life time.

\subsection{Relationship to Other Programs}

Even at these lower energies, the machine energy will be significantly higher than other machines including LHC. The construction of the machine will not be significantly affected by using this approach and therefore in terms of advantages in doing early physics, no gains are possible.

\subsection{Cost/Schedule Summary}

All estimates are based on PMB (BCE) and are given 1991 dollars. The cost of 1 layer coil magnet is based on information from Erich Willen (BNL) on the RHIC Program. Erich Willen estimates that a 1 layer SSC dipole can be operated at 5 T.

RHIC dipole: Aperture $=80 \mathrm{~mm}$; Length $=9.5 \mathrm{~m}, \mathrm{~B}=4.6 \mathrm{~T}$; Nominal cost: $\$ 13100 / \mathrm{m}(\$ 13500$ /m with SSC Cable with 5T operation) in 1991 \$.

SSC Dipole: Aperture $=50 \mathrm{~mm}$; Length $=15 \mathrm{~m}, \mathrm{~B}=5 \mathrm{~T}$; The cost $=13500 \$ * 50 \mathrm{~mm} / 80 \mathrm{~mm} *$ $15 \mathrm{~m}=126600 \$$ which is about $\$ 30 \mathrm{~K}$ cheaper than the present cost of dipole. For 9000 magnets this saves about $270 \mathrm{M} \$$ in dipole costs. The lowering of energy could save an additional $\$ 20 \mathrm{M}$ in quadrupoles and corrector magnets for a total saving of $\$ 280 \mathrm{M}$.

The major problem with the pursuit of this option is that the Collider magnets (dipole as well as quadrupole) would have to be redesigned. This could reduce any savings anticipated by lower cable costs to be totally off set.

Conclusion: The change to 1 layer dipole will reduce the peak energy of the machine to about 30 $\mathrm{TeV}$ with additional risks in field quality and therefore beam quality and life time. The approach will lead to a machine that will not be easily upgradable. The approach could save up to $\$ 300 \mathrm{M}$ when contingency and escalation are included, but these savings could be totally off set by the need to redesign all of the collider magnets. Prospects of raising the luminosity to $10^{34}$ to achieve the ultimate physics goals of the SSC program do not seem probable. With these disadvantages in mind, it seems more appropriate to consider sthe option of leaving out magnets 
during installation as this can achieve the same results in a more straight forward manner as discussed in the next section.

\subsection{Program Summary (Missing Magnets)}

In this option Collider dipole magnets of the current design are left out during the installation with the aim to replace them later. This means that the Collider would operate at a lower energy depending on the number of dipoles removed. Most of the Collider half cells are comprised of five 15-m-long dipoles and the most likely scenarios studied [1] are to remove one or two out of the five and replace them with empty cryostats. With a resulting half cell consisting of four dipoles (4/5ths-Option), the Collider CM energy would be $32 \mathrm{TeV}$, while a half cell of three dipoles (3/5ths-Option) would yield a CM energy of $24 \mathrm{TeV}$. Luminosities would also be reduced proportionately to the energy with a substantial reduction in physics reach. However, the lower beam energies would reduce the synchrotron radiation generated such that a savings in cryogenic capapcity might be realized. This savings would be off set by changes required to make the machine operate correctly, such as, the quadrupole magnets are currently arranged in series with the dipole magnets and for the same current would result in too much strength for a reduced half cell of dipoles. Therefore, the quadrupole magnets might have to be reduced in length or powered separately requiring more cryogenic power feed-thrus, cabling and power supplies.

\subsection{Physics Program}

4/5ths-Option. The physics program for a reduced CM energy of $32 \mathrm{TeV}$ would be similar to that described above for the option where coils are missing in the dipole magnets (section 4.2). Though the energy and luminosity are reduced there would remain still be a large physics reach that could discover a large mass Higgs boson. To insure the same coverage of the $40 \mathrm{TeV}$ Collider would require an increase in luminosity by a factor of two or more if the Higgs were not discovered.

3/5ths-Option. The resulting CM energy is $24 \mathrm{TeV}$ and reduced to a point where the luminositiy would have to be increased substantially to near $10^{34}$ to achieve the physics reach of the original $10^{33}-40 \mathrm{TeV}$ Collider, suggesting that it would probably be more cost effective to repace the missing dipoles than invest in a R\&D program to achieve the higher luminosities. Therefore, this program is similar to other staging options in the sense that it divides the SSC project into two successive construction periods to lower costs between now and FY99. The replacement of the missing dipole magnets to bring the beam energy up to $20 \mathrm{TeV}$ is postponed until FY2003.

\subsection{Relationship to Other Programs}

With either the $4 / 5$ ths- or 3/5ths-Options the CM energy would be significantly above other machines including LHC, though the luminosity would lead to a much reduced program of physics relative to LHC in the 3/5ths-Option. As both options lend to a staged approach leading to the full Collider physics reach at a later stage, there could be an intermediate program that would lead to full physics results at a later time.

\subsection{Cost Summary}

$4 / 5$ ths-Option. The cost savings are estimated to be about $\$ 100 \mathrm{M}$ to $\$ 200 \mathrm{M}$ in this case which includes the reduced number of dipole magnets, and the additional cost for empty cryostats to 
replace them. As noted above, there are some other savings realizable from a reduced cryogenic

heat load, but this is probably off set by the cost to redesign the quadrupole magnets to have a shorter effective length for the same dipole currents.

3/5ths-Option. The cost savings are estimated to be about $\$ 300 \mathrm{M}$ in this case which includes the reduced number of dipole magnets, and the additional cost for empty cryostats to replace them. Again, there are some other savings realizable from a reduced cryogenic heat load, off set by the cost to redesign the quadrupole magnets to have a shorter effective length for the same dipole currents. However, in this case it might be more cost effective to keep the same quadrupole design as currently planned for the Collider and install feed-thrus to allow the quadrupoles to be powered independently of the dipoles in the half cell. This is because the CM energy in the 3/5ths-Option has been reduced to an extent where achieving the physics goals of the SSC can only be achieved by replacing the missing dipole magnets later in the program to go back to 20 $\mathrm{TeV}$ on $20 \mathrm{TeV}$.

\subsection{References}

[1] M. Syphers, "Investigation of Phasing the Collider: 4/5ths Option," Internal SSCL Draft Report, 1993

\section{Asymmetric p-p collider at $2 \times 20 \mathrm{TeV}$}

\subsection{Program Summary}

In this option we consider colliding the HEB beam at $2 \mathrm{TeV}$ with the beam in a single ring from the Collider at $20 \mathrm{TeV}$. The resulting CM energy (near $13 \mathrm{TeV}$ ) is similar to that at LHC but the best estimate for the initial luminosity is $10^{32}$ and will not be competitive requiring an upgrade where the second Collider ring is added later to achieve the full physics potential of the SSC. Therefore, this program is similar to other staging options in the sense that it divides the SSC project into two successive construction periods to lower costs between now and FY99. The construction of the second $20 \mathrm{TeV}$ collider ring is postponed until FY2003. In this scenario the machine construction is divided into two phases: Phase 1 - construction of $20 \times 2 \mathrm{TeV}$ collider using HEB and one $20 \mathrm{TeV}$ collider ring; this phase should be completed by FY99. Then there will be 3 years of operation using this asymmetric collider and one detector. Meanwhile, the construction of magnets for the second ring and of the second detector will take place. Phase 2 the completion of the $20 \times 20 \mathrm{TeV}$ collider (FY2003 and FY2004).

\subsection{Physics Program}

From a physics point of view, the $20 \mathrm{TeVx} 2 \mathrm{TeV}$ asymmetric collider is equivalent to $6.3 \times 6.3$ $\mathrm{TeV}$ symmetric machine (12.6 TeV center of mass energy). This is close to the energy chosen for the LHC project (14-16 TeV in the center of mass). Not surprisingly our study has shown that in principle the physics potential of $20 \times 2 \mathrm{TeV}$ collider is close to the physics potential of the LHC at lower luminosity (we assume that the nominal luminosity of the asymmetric collider would be $10^{32}$, i.e., two orders of magnitude lower than the LHC). This is illustrated in Fig. 5.1, where the cross sections of the Higgs particle production for the SSC $20 \times 20 \mathrm{TeV}$, Asymmetric Collider 20x2 TeV and LHC $8 \times 8 \mathrm{TeV}$ are compared (in arbitrary units). We will list below our conclusions about the discovery potential of the asymmetric collider after many years of running time (Integrated luminosity of about 1 inverse $\mathrm{fb}$.).

1. Higgs can be discovered through 4 lepton decays in the mass range of $200-400 \mathrm{GeV}$. 
2. Top Quark (if not discovered at Fermilab) can be discovered in a mass range up to 200 $\mathrm{GeV}$ through both $1+\mathrm{mu}$ and $1+$ jets decay modes.

3. compositness scale limits can be pushed up to $10 \mathrm{TeV}$ using pp jets production or DrellYan processes.

4. Super symmetry can be discovered through missing Et studies or same sign dileptons studies for Gluino/Squark masses below $0.4-0.5 \mathrm{TeV}$.

\subsection{Luminosity Considerations}

The luminosity for the collision of the HEB beam with the Collider beam is given by:

$$
\mathrm{L}=\frac{\mathrm{f} \mathrm{N}_{\mathrm{HEB}} \mathrm{N}_{\mathrm{Col}}}{2 \pi \sigma^{2}}
$$

where $f$ is the frequency of revolution $\left(6 \times 10^{4} \mathrm{~Hz}\right), \mathrm{N}_{\mathrm{HEB}}$ and $\mathrm{N}_{\mathrm{Col}}$ are the number of protons in the HEB $\left(8 \times 10^{9}\right)$ and Collider $\left(8 \times 10^{9}\right)$ respectively. Sigma squared is the sum of the squares of the beam widths, which is dominated by the HEB having a mean square beam width ten times that for the collider and is given by $\sigma_{\mathrm{HEB}}{ }^{2}=\beta^{*} \varepsilon \gamma^{-1}$. A major limitation is the head-on tune shift for the HEB beam, $\Delta v_{\mathrm{HEB}}=0.004$, that can be shown places an additional, but weaker, limitation that $\beta_{\mathrm{HEB}} * / \beta_{\mathrm{Col}} *$ is less than or on the order of 0.4 . Using the values of 2130 for $\gamma$ and $\varepsilon$ equal to $10^{-4} \mathrm{~cm}-\mathrm{rad}$ for the HEB yields:

$$
\mathrm{L}=\frac{2 \times 10^{31}}{\beta^{*}}
$$

where $\beta^{*}$ is for the HEB only.

The best values imagined for $\beta_{\mathrm{HEB}} *$ are near $0.25 \mathrm{~m}$ as $\mathrm{UNK}$ designed interaction regions near this value $(0.3 \mathrm{~m})$. This would place the maximum luminosity using current parameters for the collider and the HEB at $10^{32} \mathrm{~cm}^{-2} \mathrm{~s}^{-1}$. Using strategies that had been discussed as means to increase the SSC luminosity by an order of magnitude (bunch coalescing, etc.) it might be possible to achieve a luminosity near $10^{33}$ ultimately for a $20 \times 2 \mathrm{TeV}$ collider. Higher luminosities do not seem possible.

\subsection{Machine Configuration}

It is a major technical challenge to arrange collisions between the HEB and the SSC main ring modifying the current design and noting that the LINAC, LEB and MEB are already under construction and provide a constraint on HEB placement. The HEB would have to be lowered to the same level as the Collider with the concurrent lengthening of the MEB to HEB transfer lines. The present design for the West Utility area for the collider where collisions would have to take place is very complicated with rf and beam ejection. To allow beam transfer from the HEB to the Collider and still affect collisions in a suitable collision hall, it might be necessary to move the HEB to the East by tens of meters allowing for the addition of a diamond-type by-bass system, or even move the HEB to the South to allow collisions in IR-1. Moving the HEB might necessitate purchase of additional land which might be prohibitive at present as new land acquisition would open up the Lab Environmental Impact Statement for review. Otherwise the HEB diameter and energy might have to be reduced to fit within the current site boundary. 
The redesign for the new transfer lines and HEB changes, something that at present we are not sure how to accomplish given the current injector positions, could amount to much money and perhaps degradation of HEB performance.

\subsection{Detector for the Asymmetric Collider}

A natural question about the detector is if it can be the same for the asymmetric collider as for the $20 \times 20 \mathrm{TeV}$ machine. We have performed this study and our conclusion is that the detector that is optimized for $20 \times 20 \mathrm{TeV}$ operation is suitable for $20 \times 2 \mathrm{TeV}$ collider as well, so that one can use essentially the same detector for both phases of collider operations. This conclusion is illustrated in Fig. 5.2 through Fig. 5.5. Figures 5.2 and 5.3 show that eta distribution of leptons and gammas from Higgs decays into four leptons and two gammas is not changing much when $20 \times 20 \mathrm{TeV}$ collider is replaced by $20 \times 2 \mathrm{TeV}$. In figure 5.4 one can see that energy flow for minimum bias events is asymmetric only by factor 2.5 in eta distribution within the rapidity range $-5<$ eta<+5. Next figure (Fig. 5.5) illustrates that trigger efficiency for a $140 \mathrm{GeV}$ Higgs decay into four muons (Pt-threshold of $20 \mathrm{GeV}$ for a single muon trigger or $10 \mathrm{GeV}$ for two muon trigger) drops from $88.8 \%$ in case of $20 \times 20 \mathrm{TeV}$ to $84.2 \%$ for $20 \times 2 \mathrm{TeV}$. This is a small loss which probably can be partially recovered by tuning the trigger thresholds.

\subsection{Cost Summary}

The cost savings at phase 1 is basically the cost of one $20 \mathrm{TeV}$ Collider ring which was estimated in Section 3 to be about $\$ 1.1$ to $\$ 1.4$ billion. This cost must be off set by any changes that would be incurred by moving and redesigning the HEB and the transfer lines, perhaps $\$ 200 \mathrm{M}$. or more. Given that the LINAC, LEB and MEB are already under construction and set in place, there is no current idea how such a redesign might even proceed. Furthermore, placing a detector on the West side would require the addition of a detector hall costing at least $\$ 32 \mathrm{M}$ as it would have to be constructed in the Eagleford shale with pylons for stabilizing the detector.

\section{Figure Captions}

Figure 5.1. Cross-section for $\mathrm{H}^{0}$-production as a function of $\mathrm{M}_{\mathrm{H}}$ for various center of mass energies: + SSC 20 on $20 \mathrm{TeV}$, o LHC 7 on $7 \mathrm{TeV}$, black dots SSC 20 on $2 \mathrm{TeV}$. The crosssections were generated with Pythia.

Figure 5.2. Eta-distribution of the photons produced in $\mathrm{H}^{0} \rightarrow \gamma \gamma$ at $\mathrm{M}_{\mathrm{H} 0}=140 \mathrm{GeV}$. The solid line refers o 20 on $2 \mathrm{TeV}$, the dashed line to 20 on $20 \mathrm{TeV}$. The events were generated with Pythia. Eta was extracted from the generator output, a detector was not simulated.

Figure 5.3. Eta-distribution of the muons produced in $\mathrm{H}^{0} \rightarrow \mathrm{ZZ}^{\wedge *} \rightarrow 4$ muons at $\mathrm{M}_{\mathrm{H} 0}=140$ $\mathrm{GeV}$. The solid lines refers to 20 on $2 \mathrm{TeV}$, the dashed line to $20 \mathrm{on} 20 \mathrm{TeV}$. The events were generated with Pythia. Eta was extracted from the generator output, a detector was not simulated.

Figure 5.4. Energy-flow in minimum bias p-p background events for 20 on $2 \mathrm{TeV}$ (solid line) and 20 on $20 \mathrm{TeV}$ (dashed line). The top plot shows the energy per unit of eta, per event for eta $<10$, the bottom plot shows the region of eta $<5$. 
Figure 5.5. The triggers for the process $\mathrm{H}->4$ muons are a single muon trigger with a $\mathrm{Pt}-$ threshold of $20 \mathrm{GeV}$ and a 2 muon trigger with a Pt threshold of $10 \mathrm{GeV}$. See also GEM-TN-93332.

The studies presented in GEM-TN-93-332 were repeated for $\mathrm{H}->4$ muons. Without changing the trigger thresholds and for a $\mathrm{H}^{0}$-mass of $140 \mathrm{GeV}$, the trigger efficiency drops from $88.8 \%$ to $84.2 \%$. This is a small loss and by tuning the trigger thresholds we can probably recover some of these events. 


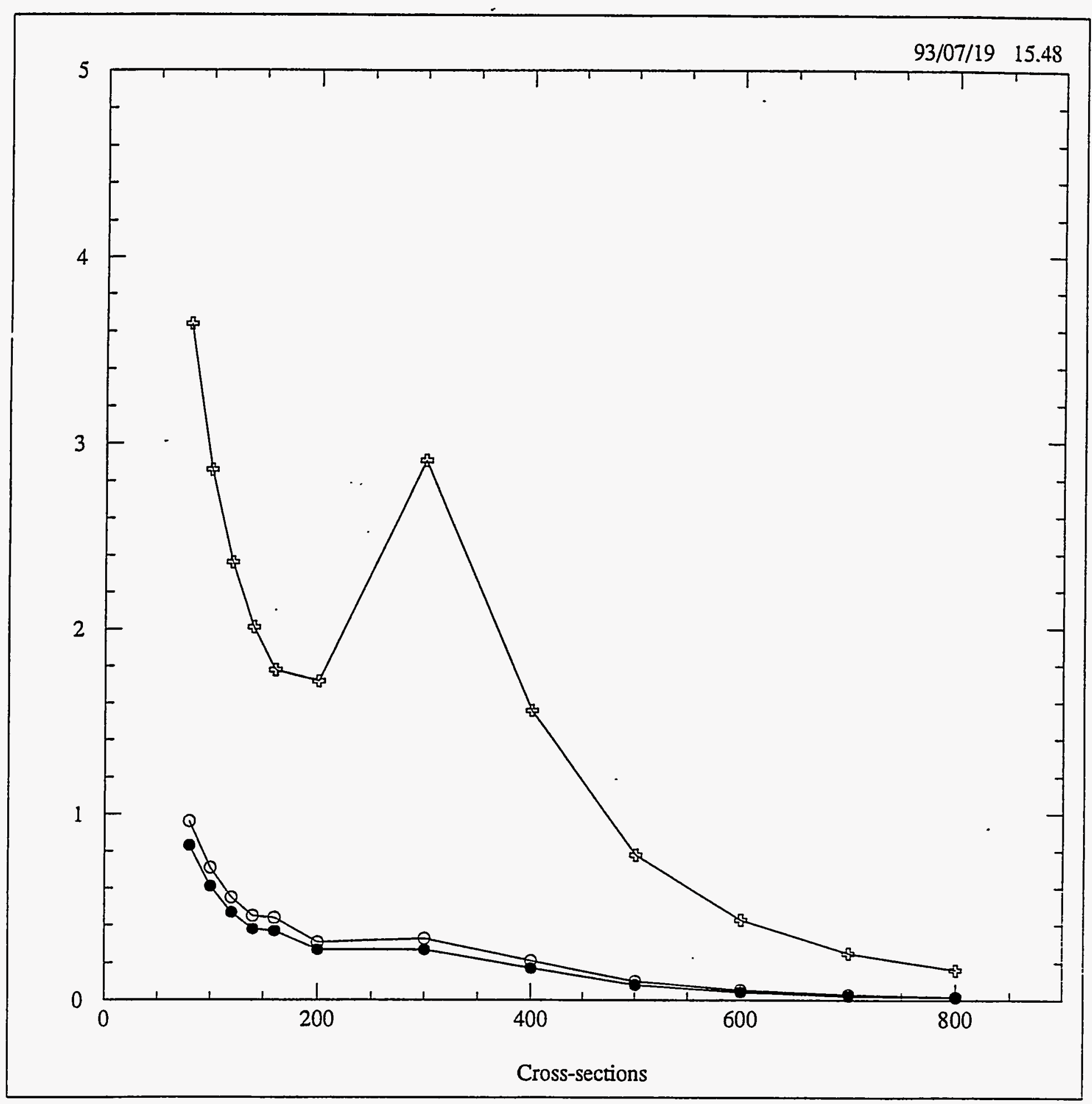



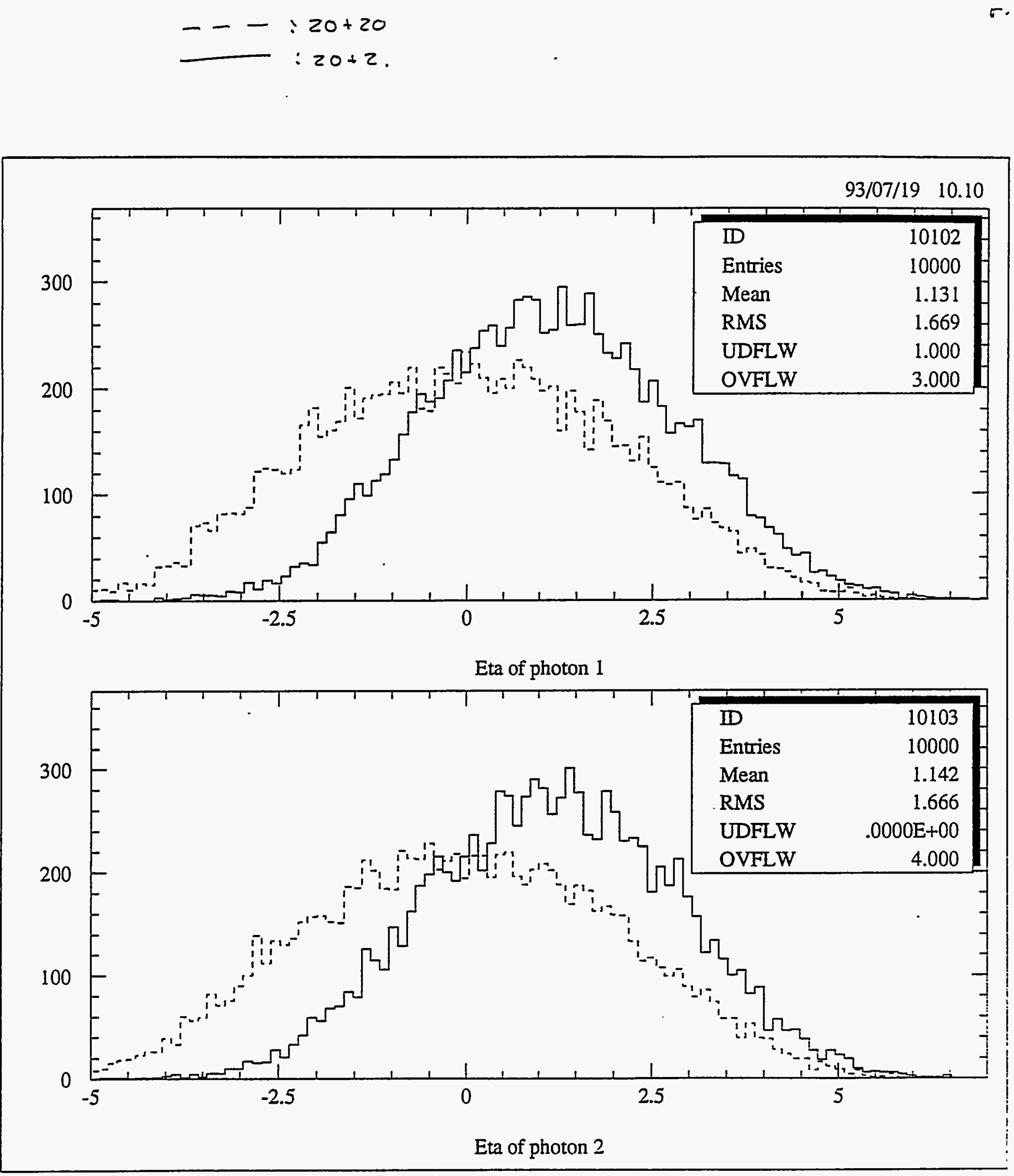

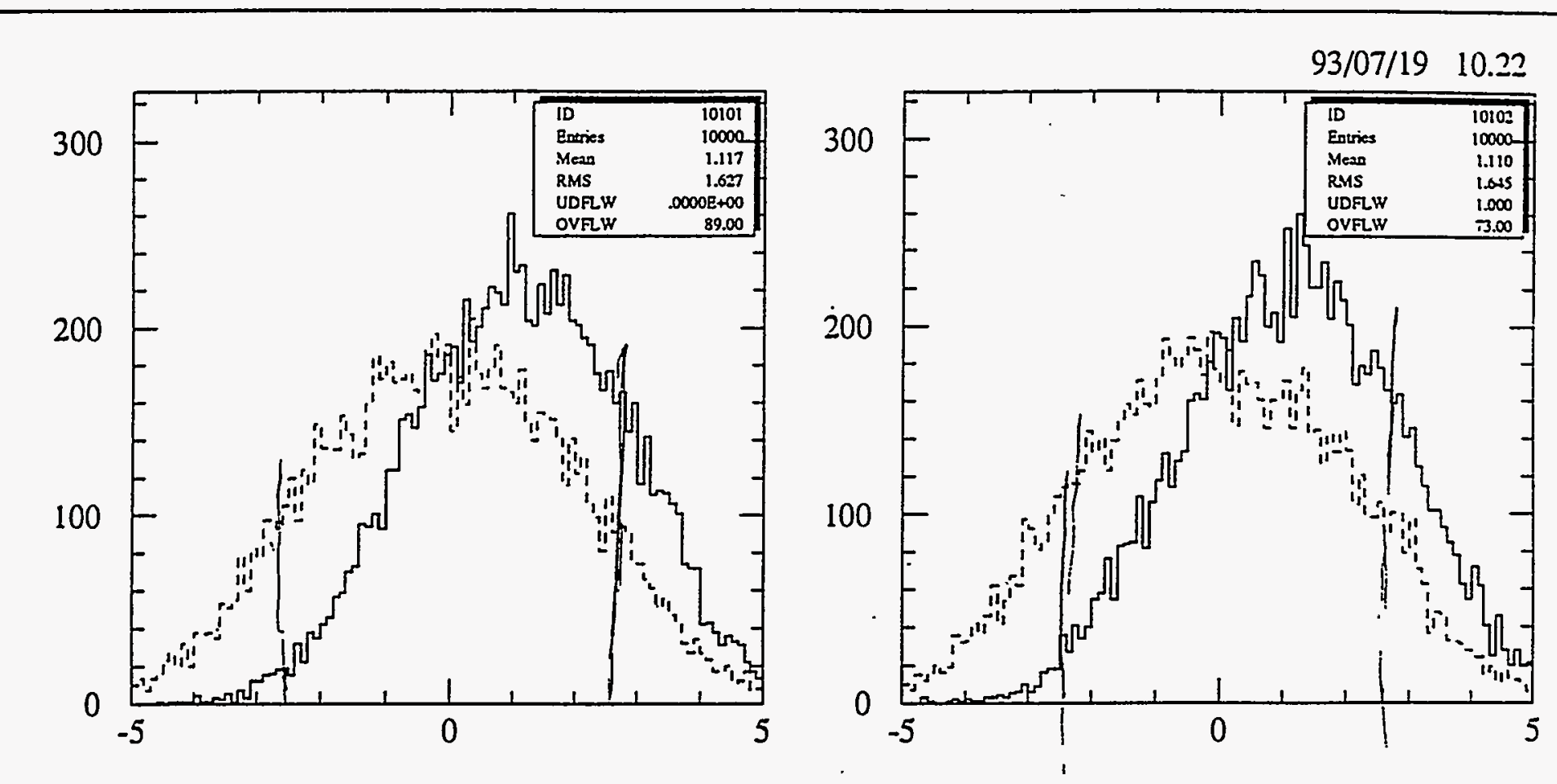

Eta of muon 1

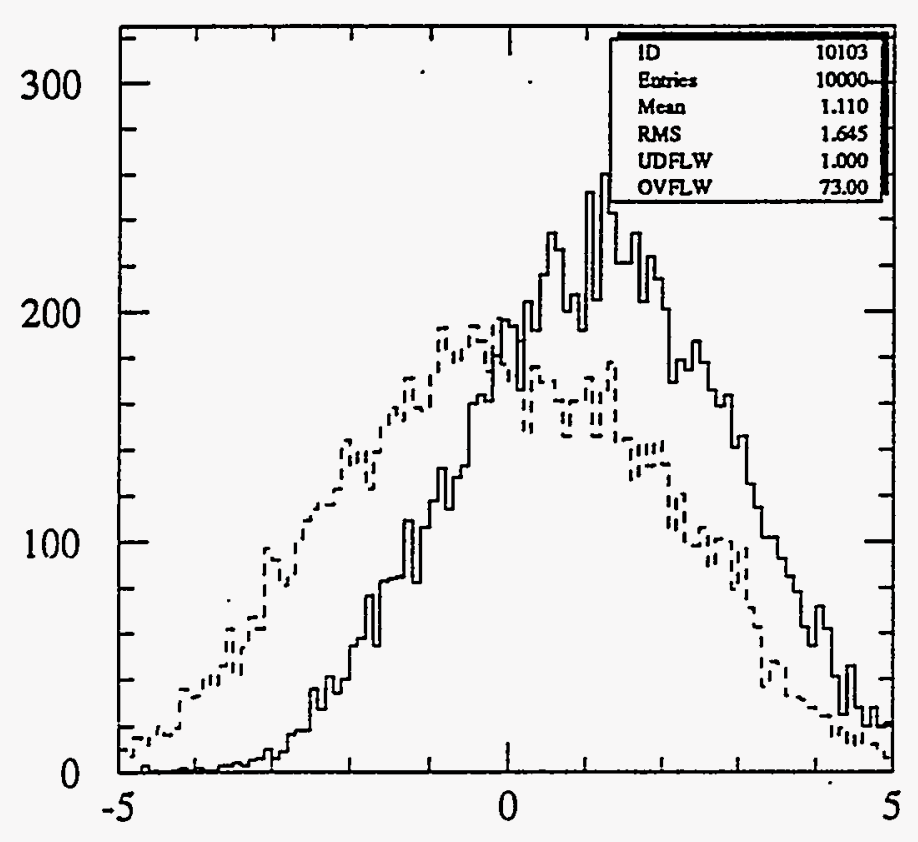

Eta of muon 3

Eta of muon 4 
$93 / 07 / 19 \quad 10.22$

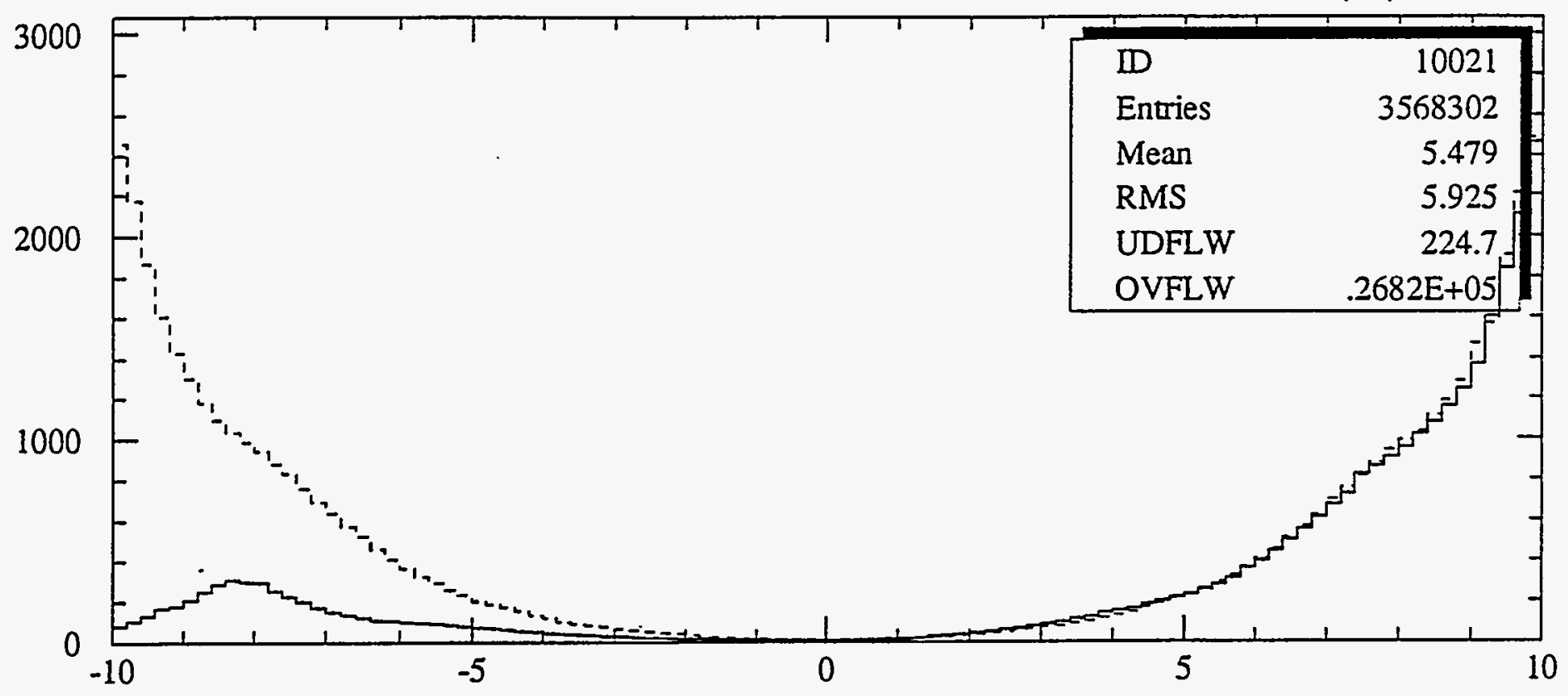

Energy/eta/event

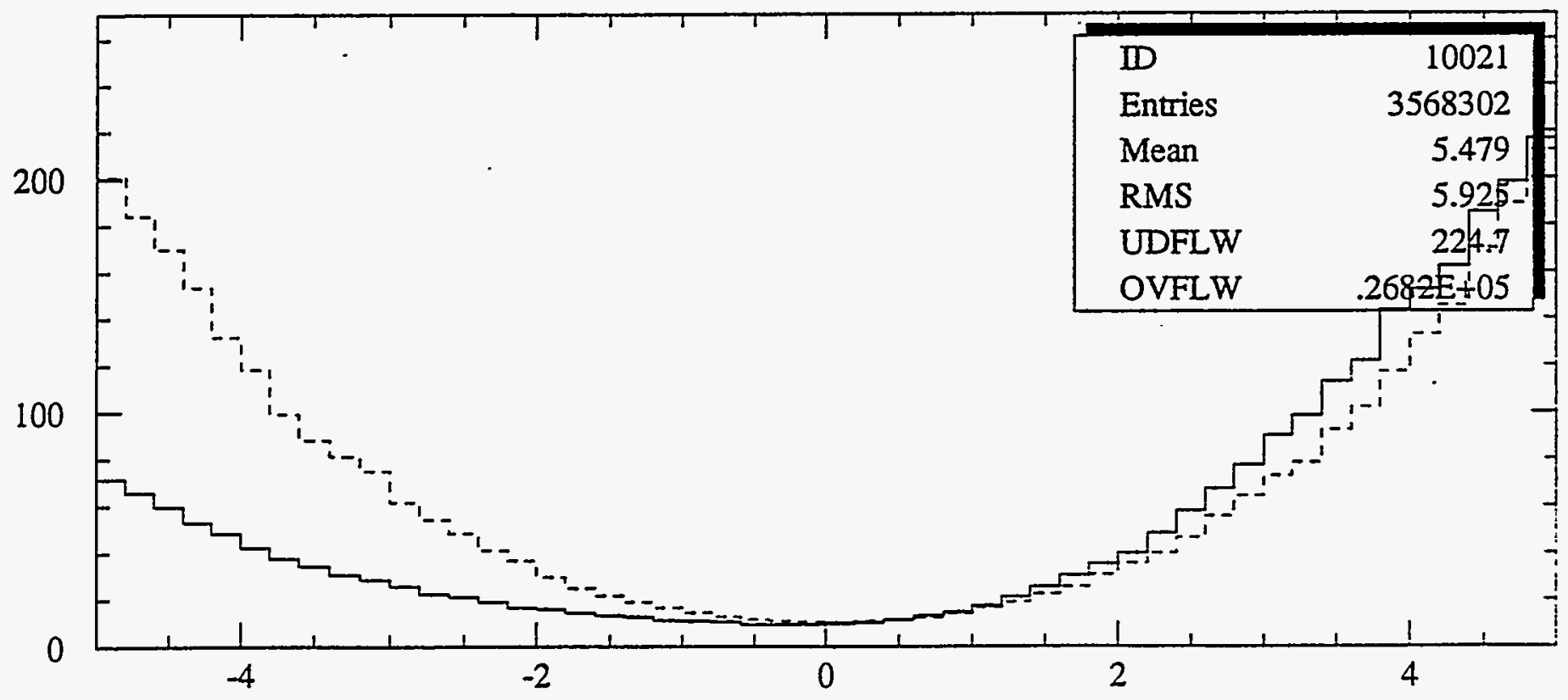

Energy/eta/event 


$$
H \rightarrow 4 \mu
$$

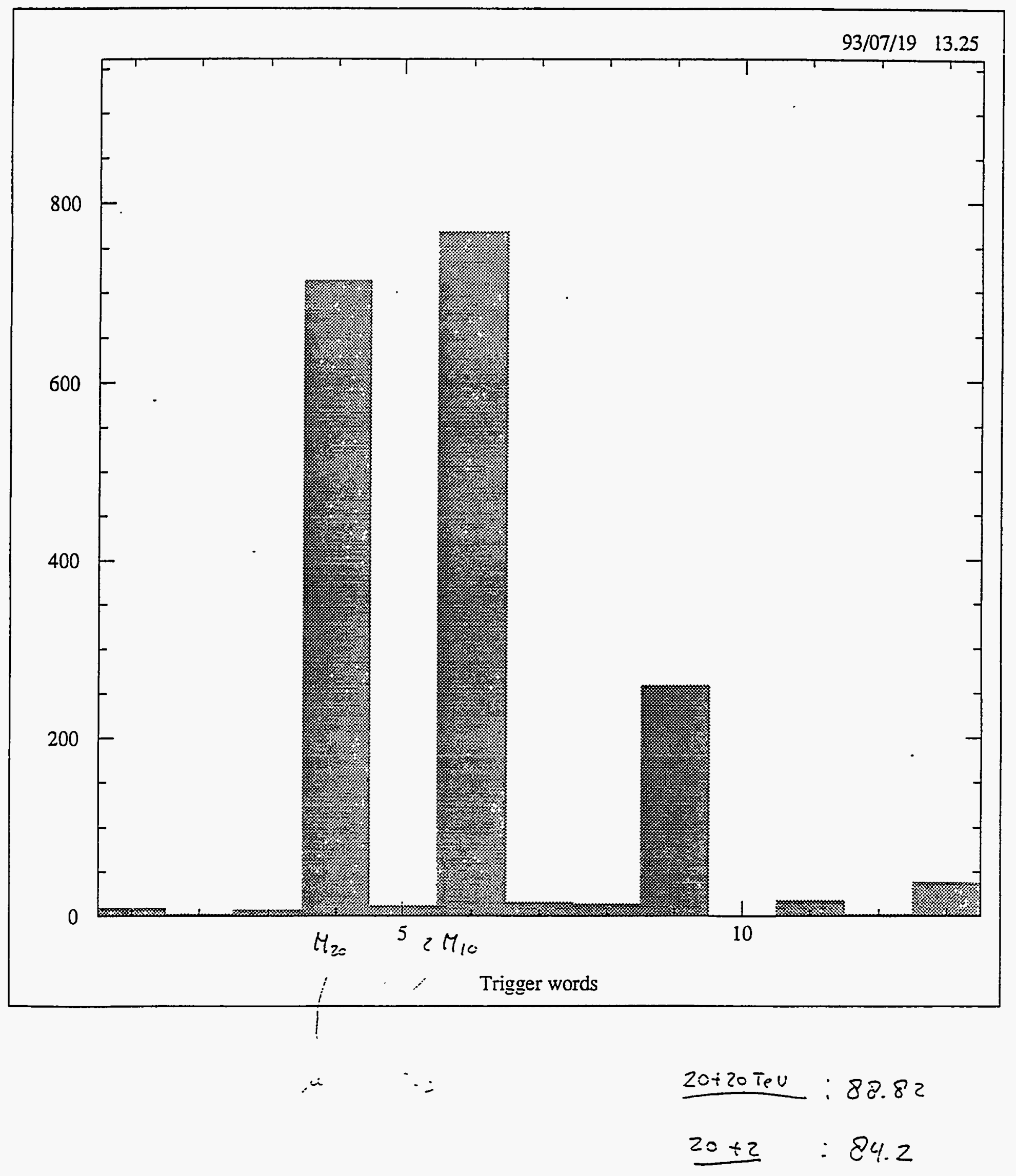




\section{P-p collider at $2 \times 2 \mathrm{TeV}$}

\subsection{Program Summary}

This program concept divides the SSC project into two successive construction periods to lower costs between now and FY93. The $20 \times 20$ Collider construction is delayed until FY98. The machine evolution is divided into two phases: Phase I - all injector machines through the HEB are completed, and a pair of intersecting proton rings are installed in the HEB tunnel by FY98; Phase II - the $20 \times 20$ Collider is constructed between FY99 and FY04.

A program of low energy p-p collider physics is initiated at completion of the HEB and carried out during Phase II Collider construction. Low-level R\&D is pursued on the Phase II Collider during Phase I. Advantage is taken during the fiscal years 1994-1997 of the lower costs of the injector machines relative to the Collider to maintain the SSC cost profile at or below $\$ 500 \mathrm{M}$ per year in FY94 dollars. Operational experience with the injector string will provide a more rapid rise to full luminosity in the Phase II Collider.

\subsection{Physics Program}

The pair of $2 \mathrm{TeV}$ proton rings in the HEB tunnel could be made to collide that will provide the highest energy and intensity collisions available until completion of the beam Phase II Collider. The maximum luminosity this machine can achieve is near $10^{33} \mathrm{~cm}^{-2} \mathrm{sec}^{-1}$ (Figure 2.3.1). The maximum energy achievable in the planned tunnel is about $2.7 \mathrm{TeV} / \mathrm{beam}$, based on reasonable extrapolation of existing magnet technology. A larger radius tunnel could be constructed, (see Figure 6.1) but such new land acquisition would open up the Lab Environmental Impact Statement for review. A new EIS is a lengthy process that probably prohibits this possibility.

Given a center of mass energy in the $4 \mathrm{TeV}$ range, and luminosity in the $10^{33}$ range, the capabilities of such a machine can be quantified (see section 2). The main physics goals of such a machine are to study top, and extend SUSY limits to the several hundred GeV mass range. No aspect of the MSM Higgs Sector is expected to be accessible. Possible charged Higgs decays of the top quark are also not fully accessible.

The main competition to this machine comes from the main injector upgrade at FNAL. AT 2 $\mathrm{TeV}$ and $10^{32}$ luminosity an upgraded FNAL provides direct competition for the same high pt physics in an existing set of experiments. In addition, possible completion of the LHC on a similar time scale would threaten to make this machine obsolete (see Table 6.1).

Table 6.1. Comparison of $\mathrm{TeV}$ - Class Colliders

\begin{tabular}{|l|c|c|c|l|}
\hline Machine & CM E (TeV) & L (cm $\left.\mathbf{c s e c}^{-1}\right)$ & $\begin{array}{c}\text { Turn on } \\
\text { Date }\end{array}$ & Comments \\
\hline Tevatron & 2.0 & $10^{31}$ & FY92 & Exists now with detectors \\
Tevatron & 2.0 & $10^{32}$ & FY98 & $\begin{array}{l}\text { Completion date of upgrade } \\
\text { uncertain } \\
\text { upgrade }\end{array}$ \\
HEB & 4.0 & $10^{33}$ & FY98 \\
LHC & 15.0 & $10^{34}$ & $?$ & $\begin{array}{l}\text { Pre-proposal stage } \\
\text { Pre-proposal stage }\end{array}$ \\
\hline
\end{tabular}


There would also exist opportunities for b-physics and other low-pt experiments at such a machine.

\subsection{Relationship to Other Programs}

Fermilab is in the midst of the top quark discovery search at this writing. The upgrade program should provide enough luminosity to perform credible top studies if the top quark lies in the $\leq$ $150 \mathrm{GeV}$ mass range. If the top quark is more massive than expected ( $>200 \mathrm{GeV})$, detailed top studies may await the BSC.

The LHC future is uncertain, and it may or may not be constructed on a similar time scale. If the LHC is constructed by $\sim$ FYO0, the BSC would be completely outclassed. Thus, the "window of opportunity" for the BSC is quite narrow-bracketed on the low side by the Tevatron upgrades, and on the high side by the LHC. Delays in the Tevatron and/or LHC programs relative to the BSC would widen the window for its useful operation.

\subsection{Cost/Schedule Summary}

This option requires a second ring to be installed into the HEB tunnel along with an interaction region, detector hall and a new detector. This cost could be as much as $\$ 600 \mathrm{M}$ and would yield a program far short of the SSC physics objectives. Therefore, this option is sensible only in the staging context in which the final $20 \times 20 \mathrm{TeV}$ Collider appears at a later stage. 


\section{Asymmetric p-p collider at $0.2 \times 2 \mathrm{TeV}$}

\subsection{Program Summary}

In this option, the SSC is completed up through the High Energy Booster in phase one. Collisions can occur between the HEB and the Medium Energy Booster, at energies consistent with beauty production. A luminosity of approximately $10 \times 10^{32} \mathrm{~cm}^{-2} \mathrm{~s}^{-1}$ is possible. A new detector hall is built around the interaction point. The short-term cost is nearly zero, with the costs of the interaction region being offset by savings in the HEB civil construction. The final collider program costs would be increased by approximately $25-50 \$ M$ due to increased costs in the HEB to Collider transfer lines.

This option would provide many years worth of very good b-physics while allowing work to proceed on the Collider. This facility would only be surpassed by the colliding beams at Fermilab, and new, dedicated beauty factories, such as the one planned for KEK and the possible US facility.

\subsection{Machine Configuration}

The changes to the present facility required for this option are relatively simple and straightforward. The most dramatic of these changes is that the HEB would need to be repositioned to be at the MEB level and moved somewhat southeast from its present location. This would result in a considerable immediate cost reduction in the HEB civil construction due to the much shallower shafts required for a near-surface machine. This cost reduction would approximately offset all of the costs associated with producing MEB - HEB collisions, producing a high-luminosity interaction region, and providing a small interaction hall.

Some modifications would be required in the MEB lattice and more extensive changes would be required in the HEB design in order to bring the two beams into collision and produce a suitable low-beta region. This would necessitate the introduction of crossing magnets in the two rings as well as low-beta quadrupoles. Collisions would occur at the location at the present CW transfer line from the MEB to the HEB. Both machines are currently configured with little or no dispersion at these locations. There is ample space within these regions to produce a beam crossing and a beta star of approximately 1 meter. This would give a maximum luminosity of 2 $\mathrm{x} 10^{32} \mathrm{~cm}^{-2} \mathrm{~s}^{-1}$ at a center-of-mass energy of $1.27 \mathrm{TeV}$, highly boosted in the forward direction.

Operation of the HEB at the MEB level would pose no problems. Shielding, etc., would be sufficient for such a change. Difficulties would arise in the civil construction for the HEB. The abort lines would need to be reconsidered in order to take the beams to the present beam dump locations. More likely, new locations and fee-simple land acquisitions would be desired. More serious difficulties would occur in constructing the future transfer lines to the Collider. The placement of straight sections in the collider would need to be modified in order to accommodate the altered geometry, and the transfer lines would be considerably longer. In addition, the transfer lines would need to bend the beams both horizontally and vertically, as opposed to the present, purely vertical displacement. This would require adding a considerable amount of strong horizontal bending the current lines. A rough estimate of the cost increase is 25 - 50 \$M, depending on the detailed design. Some of this cost increase would be offset by a reduction of the cost of the MEB to HEB transfer lines. These would be somewhat shorter and contain no large vertical dogleg. The amount of this cost reduction has not been estimated.

\subsection{Physics Program}


The physics program available at this facility would surpass any present proposals excluding dedicated $b$ factories. Below is a table giving a comparison of this option with present proposals:

\begin{tabular}{|c|c|c|c|c|c|c|}
\hline & $\begin{array}{l}\text { HERA } \\
\text { Wire }\end{array}$ & $\begin{array}{l}\text { HEB } \\
\text { Wire }\end{array}$ & $\begin{array}{l}\text { LHC } \\
\text { Crystal }\end{array}$ & $\begin{array}{l}\text { Tevatron } \\
8 \times 1000\end{array}$ & $\begin{array}{c}\text { SSC } \\
\text { Crystal }\end{array}$ & $\begin{array}{c}\text { MEB } \otimes \text { HEB } \\
200 \times 2000\end{array}$ \\
\hline$\sqrt{\mathrm{s}}[\mathrm{GeV}]$ & 43 & 61 & 115 & 178 & 193 & 1265 \\
\hline$L\left[\mathrm{~cm}^{-2} \mathrm{~s}^{-1}\right]$ & - & - & - & $9 \times 1030$ & - & $2 \times 1032$ \\
\hline$\sigma_{b} \vec{b}[\mu b]$ & $0.008-0.016$ & $0.16-0.32$ & $0.6-1.0$ & 1.65 & 2.0 & 27 \\
\hline $\mathrm{b} \overline{\mathrm{b}} / \mathrm{sec}$ & $24-48$ & $480-960$ & $605-1009$ & 15 & 1593 & 5400 \\
\hline
\end{tabular}

\subsection{Physics Potential of MEB-HEB Collider}

An asymmetric collider based on colliding protons from the Medium Energy Booster (MEB) and High Energy Booster (HEB) would have an energy of $\sqrt{s}=1265 \mathrm{GeV}$ and an estimated luminosity of $L=2 \times 10^{32} \mathrm{~cm}^{-2} \mathrm{~s}^{-1-}$. This should be compared with the Tevatron, which is expected to have $\sqrt{\mathrm{s}}=2000 \mathrm{GeV}$ and $L=10^{32} \mathrm{~cm}^{-2} \mathrm{~s}^{-1-}$ after completion of the Main Injector and upgrades to the collider ring. The rough rule of thumb is that for high-mass physics a factor of 2 in energy is worth a factor of 10 in luminosity. Thus, even if one were to take seriously the factor of 2 difference in the nominal luminosities, it is clear that the upgraded Tevatron is far superior for top and possible new, high-mass physics.

The cross section for $b$ production calculated ${ }^{1}$ to the next to leading order (NLO) in perturbative QCD for $m b .=4.75 \mathrm{GeV}$ with the CTEQ parton distributions is shown in Fig. 1. The cross section for $\mathrm{b} \overline{\mathrm{b}}$ production at the MEB-HEB collider is about $25 \mu \mathrm{b}$, corresponding to $5 \times 10^{10} \mathrm{~b} \overline{\mathrm{b}}$ pairs per year. Since the $b$ mass is low, the cross section is much less energy dependent than than for top, changing by less than a factor of 2 between $1265 \mathrm{GeV}$ and $2000 \mathrm{GeV}$. An asymmetric collider also has an advantage both for $b$ vertex determination and for lepton identification and triggering. This is a fairly small effect: the center of mass of the MEB-HEB collider has a rapidity $y \approx 1.05$ in the lab frame, and the $b$ cross section only decreases by about $15 \%$ from $y=0$ to $y=1.05 .1$ 
The primary goal of $b$ physics is to measure $C P$ violation in the $b$ system. In particular, the $C P$ asymmetries for neutral $B$-meson decays into $C P$ eigenstates can be related to the CKM parameters with only small uncertainties from strong interaction effects. Examples of such modes include:

$$
\begin{aligned}
& \mathrm{B}^{\circ} \rightarrow \pi^{+} \pi^{-} \\
& \mathrm{B}^{\circ} \rightarrow \varphi \mathrm{K}_{\mathrm{s}} \\
& \mathrm{B}^{\circ}{ }_{\mathrm{s}} \rightarrow \varphi \phi
\end{aligned}
$$

(One does have to worry about the contribution of penguin diagrams in some of these modes.) Unitarity of the CKM matrix can be expressed as a triangle relation among its elements in the complex plane, and the asymmetries for the reactions in Eq. (1) measure the three angles $\alpha, \beta$, and $\gamma$ respectively of this triangle. This is perhaps the cleanest way of showing that the CKM matrix is actually the origin of $C P$ violation. The $C P$ asymmetries are $210 \%$. However, the branching modes for these modes are very small, and it is necessary to tag the initial $B$ or $\bar{B}$ by determining whether $a \bar{B}$ or $B$ was produced in association with it. The combination of large asymmetries and tiny branching ratios is well matched to a hadron collider experiment.

The general conclusion of the recent Snowmass Workshop 2 is that measurement of the angle $\beta$ from the asymmetry in $\mathrm{B}^{0} \rightarrow \varphi \mathrm{K}_{\mathrm{S}}$ is feasible at the Tevatron Collider with the Main Injector and therefore also at a MEB-HEB Collider. Measurement of the angle $\alpha$ from $B^{\circ} \rightarrow \pi^{+} \pi^{-}$is considerably more difficult both because of the trigger and because of combinatorial background. These problems would be similar at the two accelerators.

Besides the basic measurement of modes like those in Eq. (1); one wants to measure $C P$ violation in other modes which are less precisely calculable but still interesting, to search for rare $B$ decays, and to study $b$ baryons and exotic $b$ particles.

The detector requirements for all of this physics include excellent $b$-vertex identification, mass resolution, and lepton identification, and perhaps $\pi / K / p$ identification. All of these are intended to reduce the combinatorial background to the $B$ decays. Plans exist to upgrade CDF and D O with improved vertex detectors and electronics to operate at higher luminosity, both for top physics and for $B$ physics. A new experiment at the MEB-HEB collider dedicated to $B$ physics might include specialized trigger hardware or $\pi / K / p$ separation that gave it an advantage, but it would have to compete for resources with other experiments.

In summary, the MEB-HEB collider is interesting primarily for $B$ physics, and it provides a major gain only if the upgrade plans for the Tevatron are not carried out. Since the upgraded Tevatron is clearly superior for top and other high-mass physics, it is the preferred solution.

References:

1. A. Zieminski, in Sumamary Talks for the Workshop on B Physics at Hadron Accelerators, (unpublished).

2. ibid., talks by H. Jawahery and by F. Dejongh. 


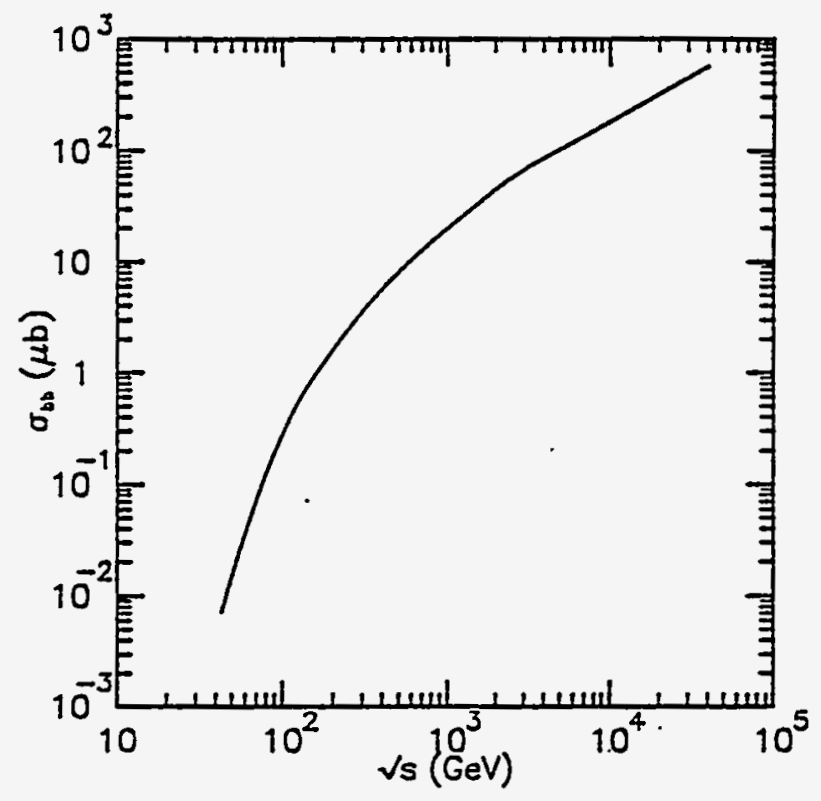

Figure71: $\sigma_{b \vec{b}}$ vs. $\sqrt{s}$ using the NLO cross section and the CTEQ parton distributions. From Ref. 1. 


\subsection{TeV Fixed Target Program}

\subsection{Program Summary}

This prgram concept divides the SSC project into two successive construction periods to lower costs between now and FY98. The $20 \times 20 \mathrm{TeV}$ Collider construction is postponed until FY98. The machine evolution is divided into two phases: Phase I - all the injector machines through the HEB are completed by 1998; Phase II - the $20 \times 20 \mathrm{TeV}$ collider is constructed between FY99 and FY04.

A program of fixed-target physics is initiated at completion of the HEB and carried out during the Phase II Collider construction . Low-level R\&D on the Collider is also pursued during Phase I. Advantage is taken during the fiscal years 1994-1997; of the lower costs of the injector machines relative to the collider, to maintain the SSC cost profile at or below $\$ 500 \mathrm{M}$ per year in FY94 dollars.

\subsection{Physics Program}

The high intensity, rapid-cycling $2 \mathrm{TeV}$ proton beam from the HEB could supply primary beam for a superior program of fixed target physics. The likely areas of maximum benefit include neutrino physics (including prompt neutrino beams and neutrino oscillations) and bottom quark spectroscopy. The existing competition is the Tevatron at Fermilab (due to reach $1 \mathrm{TeV}$ before 1998) with its existing and fully-deployed fixed target physics program. A possible future competitor would be a fixed-target program at UNK in Russia. The UNK machine is not complete at this time and prospects for its completion and use as a fixed target physics facility are dim.

The relative machine/beam capabilities for a fixed-target physics application, of the competitive proton accelerators are shown in Table 8.1.

Table 8.1. Comparison of TeV-Class, Fixed-Target Proton Accelerator

\begin{tabular}{|c|c|c|c|c|}
\hline Machine & $\mathrm{E}(\mathrm{TeV})$ & I (p/cycle) & $\mathbf{I}(\mathrm{p} / \mathrm{yr})$ & Comments \\
\hline Tevatron ${ }^{\mathrm{a}}$ & 1.0 & $3 \times 10^{13}$ & $6 \times 10^{18}$ & $\begin{array}{l}\text { exists, will reach } 1.0 \mathrm{TeV} \\
\text { by } 1996\end{array}$ \\
\hline $\mathrm{UNK}^{\mathrm{b}}$ & 3.0 & $3 \times 10^{13}$ & $3 \times 10^{18}$ & $\begin{array}{l}\text { incomplete, uncertain future } \\
\text { prospects }\end{array}$ \\
\hline SC HEBC & 2.0 & $2 \times 10^{13}$ & $1 \times 10^{18}$ & pre-proposal stage \\
\hline
\end{tabular}

a. Tevatron parameters reflect recent fixed-target operating experience.

b. UNK parameters are taken from the UNK design study.

c. SSC HEB parameters are taken from the SSC design study.

The secondary beam characteristics depend, of course, on the chosen program of physics. Here, we indicate a few of the possibilities that could be realized for the 2 TeV HEB fixed-target application and compare them with the competitors.

In Figure 8.1, prompt and conventional neutrino beam fluxes are compared, assuming all the accelerated protons are used for such a beam. If the chosen physics program requires beam 
sharing, the flux will be lower by the shared fraction. From this plot, we see that for all but the highest neutrino energies in the conventional neutrino beams, the Tevatron proton flux outbalances the energy advantage of the SSC-HEB. The UNK parameters assumed indicate this machine could complete well with the others in conventional neutrino physics. For prompt neutrino (beam-dump) beams, higher energy is a significant advantage because of the rapidly growing cross-section for heavy quark production. In this case, the UNK parameters indicate a clear advantage for this machine; the SSC-HEB does better than the Tevatron only at the highest neutrino energies.

A $2 \mathrm{TeV}$ extracted beam from the HEB could in principle be capable of producing a very intense and energetic neutrino beam. The neutrino intensity could potentially be 10-20 times higher than the Fermilab neutrino beam delivered in the past. The average energy of the neutrino will be about $400 \mathrm{GeV}$, with a spectrum ranging from $20 \mathrm{GeV}$ up to $1500 \mathrm{GeV}$ up to $1500 \mathrm{GeV}$.

Based on the measured rates at the FNAL experiments, it should be possible to accumulate 40-80 million neutrino charged current events with a 1000 ton detector. With this impressive statistical precision, one could address questions that were only of academic interest in previous experiments and laboratories. Some of these are:

Oscillation: With an appropriate apparatus one could aim at muon-neutrino to $\tau$-neutrino oscillation with a sensitivity approaching $10^{-5}$. It follows that a corresponding sensitivity in electron-neutrino to $\tau$-neutrino will be of the order $10^{-3}$.

Right Handed Currents: The sensitivity for right handed W-bosons (charged weak currents) would be enhanced by more than an order of magnitude.

Light Neutral Heavy Lepton: Neutral heavy leptons may couple to the $\tau$ but not the $\mathrm{Z}^{\circ}$ and thus may evade detection at LEP and elsewhere. The sensitivity for such lepton with masses below a few $\mathrm{GeV}$ could be more than an order of magnitude higher at the SSC.

Weak Mixing Angle: Continuing the quest for ever higher precision of $\sin ^{2}(\theta \mathrm{w})$, the neutrino experiment at the HEB will be able to attain a precision of 0.001 . This determination of different is $\sin ^{2}(\theta w)$ from and complementary to measurements at LEP. Thus, the $\rho$ parameter of the Standard Model could be determined to about $0.25 \%$ precision. Of course some of this reasoning will weaken once we know the mass of the top quark.

Quantum Chromody namics: Some of the most compelling tests of QCD could be accomplished with HEB neutrino beam. These tests, unique to neutrino interactions due to the parity violating amplitude, will be undertaken with a precision and built in redundancy in the measurement with the aim of challenging the theory, and not just conforming it. The measurable quantities will be $F S, x F 3, q$ (bar), and longitudinal structure functions.

It remains to be seen which of these studies can be performed at HERA or at LEP / SLC or will be addressed in the low energy, high intensity $\mathrm{v}$ experiments planned for Fermilab. It is assumed that the $\tau$ neutrino will have been observed at CERN or Fermilab in one of the recently approved experiments. Once could consider a similar experiment at the SSC Collider, using either the neutrino flux from the IP or the beam dump.

Figure 8.2 shows heavy ( $c$ and $b$ ) quark production for a proton beam incident on a 0.1 nuclear interaction length target for each of the machines in Table 8.1. Fluxes of these quarks are for a nominal one-year of running, assuming a beam intensity of $10^{8}$ per second on the target with an overall $50 \%$ operating efficiency factor. This intensity was chosen under the assumption that the 
primary physics interest would be in heavy quark production dynamics and spectroscopy and that experiments would involve full-acceptance spectrometers and reconstruction of many-body final states into their parent mesons and baryons.

The physics opportunities are primarily limited to spectroscopy of baryon states or meson states that are strongly inhibited in $\mathrm{e}^{+} \mathrm{e}$ - production, along with the study of strong production dynamics for heavy quarks. This is not a virgin field given the Tevatron program of some 10 years duration, but opportunities can probably be found for useful advances using a carefully thoughtout detector and a carefully crafted program of experiments. Again, this paper doesn't propose to develop the detailed arguments for particular experiments.

It is conceivable (but highly unlikely) that fixed-target physics of light quarks could also be found for which niche experiments using the SSC-HEB would be competitive. Given the existence of a fully-developed and well-exploited physics program at Fermilab and the saturation of light quark production at low CM energies, the prospects are dim and will not be considered further here.

\subsection{Relationship to Other Programs}

Fermilab has run an $0.8 \mathrm{TeV}$ program of fixed-target physics from 1985-1991 with about half the scheduled time of Tevatron operations devoted to fixed-target running. Fermilab is also presently embarked on an improvement program that will raise the Tevatron proton beam energy to $1.0 \mathrm{TeV}$ and the accelerated beam intensity to $3 \times 10^{13}$ per cycle. Given this history, plus the fact that b-quark production cross-sections are already above the rapid rise S-value in the CM, an improvement of a factor 1.4 in the CM energy is not a very competitive edge for the SSC-HEB. Furthermore, (as presently scoped), the HEB's cycle time and capability for accelerating protonsper-year will put it at a disadvantage for neutrino physics (but probably not for hadron physics).

Finally, the cost of developing a fixed-target experimental area and creating one or more fixedtarget experiments at SSC must be weighed against the fact that Fermilab could counter the HEB program with significantly less-expensive upgrades and faster time schedules. Prospects for a physics competition with Fermilab in the area of fixed-target physics in the late 1990s are dim.

In the case of UNK, the competitive situation (which nominally favors the Russian accelerator), must also take proper account of the prospects for completing the UNK machine and devoting it to fixed-target physics. Present indications are that the pace of completion of the accelerator is very slow, the plans for a physics program are not advancing and overall prospects for a competitive program relative to Fermilab or SSC-HEB are very remote. On the other hand, if a physics goal of compelling attraction appeared, that could be best performed by a fixed-target, multi-TeV proton accelerator, UNK could be developed as a formidable competitor. This scenario would probably involve strong international participation in both the accelerator and in the experimental program in order to succeed.

Regardless of how the physics attraction of a $2 \mathrm{TeV}$, fixed-target program evolves, the SSC-HEB is not likely to be a competitive winner. It is limited by the accumulated and prospective Tevatron program at somewhat lower S-valves and by the prospects of the UNK accelerator at higher S-values.

In addition to the questionable competitive position relative to the physics programs possible at Fermilab and to the superior intrinsic physics prospects at UNK, the SSC-HEB fixed-target program faces some specific drawbacks and problems in the context of the baseline SSC Project Plans. We identify these next. 
The most obvious drawback is the fact that no $2 \mathrm{TeV}$ external beam facilities or experimental areas are planned as part of the SSC baseline. Also, it is highly unlikely that detectors useful for such a program would be later applicable in the $20 \times 20 \mathrm{TeV}$ collider program. For these reasons, new facilities costs of $\$ 100 \mathrm{M}$ would have to be added to the program cost estimate. The costs of the detectors have not been included since, if the physics program were found to be competitive, the detector would likely be funded from base program funds in the ongoing U.S. HEP program.

A second drawback is the slower nominal ramp rate of the HEB relative to the Tevatron. This represents a problem primarily for a neutrino program, since a hadron program would use very long flattop spills, diminishing the importance of ramp rate.

\subsection{Test Beam Program for Detector Development at $2 \mathrm{TeV}$}

In light of the present uncertainties regarding the best approach to completing the SSC injector complex relative to completing the collider, it is relevant to discuss the purpose and importance of test beams at the SSC. It has long been recognized that while test beam facilities are a necessary component of the injector complex, scheduling and energy constraints would require facilities at other laboratories such as FNAL in order to conduct detector component research, development, prototyping, and calibration. The relationship between the SSC test beams program and test beams programs conducted eisewhere was designed to be complementary and not competitive, but this distinction has become somewhat blurred because of the current budget and schedule uncertainties. Thus, the intent of the present discussion is to reiterate the vital role of the SSC test beams program, and to indicate where it fits into a staging or replanning of the overall SSC program.

The purpose of the SSC test beams program is to provide a means for calibrating and bench marking detector subsystems before the collider is commissioned, to provide a test bed for longterm stability and response studies once the collider is operating, and to support detector development and upgrades throughout the life of the SSC physics program. Unfortunately, hypothetical plans to stretch the schedule by delaying the construction, commissioning, and operation of the various accelerators has cast doubt on exactly when, and how much before collider operation, the SSC test beams will be available. This is a serious issue that should not be regarded as peripheral to the completion of the injector complex, but which needs to be resolved based on what is best for the detector programs.

There should be no question as to whether the SSC needs to begin test beams operations several years before collider commissioning is completed. Indeed, it is the stated position of the GEM collaboration that the completed central tracker must be tested at the SSC, and their schedule calls for testing to begin at least 3 years prior to collider operation. Although test beams at FNAL offer important opportunities in the mid-1990's, there are significant differences, detailed below, between the FNAL and SSC programs that compel an aggressive pursuit of the SSC test beams program. There has been no technical development that has eliminated or diminished the important contribution of the SSC test beams program to the ultimate success of the SSC physics program.

One of the most important aspects of the test beams program for both detector collaborations is calorimeter calibration. Calorimeter response as a function of energy is inherently nonlinear and not easily modeled. Thus, extensive calibration data is required for the calorimeter to provide unambiguous evidence of missing momentum signatures due to, for example, Higgs or supersymmetric particles. The range in energy of test beam particles necessary for an accurate calibration depends on particle type, but is approximately $2 \mathrm{GeV}$ to a few TeV. The SSC MEB test beams will provide a range of $2-170 \mathrm{GeV}$, the FNAL test beams will provide approximately 
15-850 GeV, and a possible upgrade at the SSC could furnish test beams from the HEB from about $100 \mathrm{GeV}$ to $1.8 \mathrm{TeV}$. Since both the low and high energy limits are crucial to successful calorimetry, using only the range available at FNAL will not be sufficient to answer all the questions that will arise.

The availability of beam at FNAL is a major concern. Test beams for GEM and SDC at FNAL will be available only during the limited time devoted to fixed-target experiments. A large amount of beam time will be required because both detector collaborations intend to conduct detailed testing of tracker components, calorimeter calibration, test and calibration of spare modules, and pile-up studies. The facility at FNAL will not be able to provide sufficient running time, whereas the facility at the SSC, being dedicated to SSC priorities, will be able to satisfy the requirements for the extensive amount of testing. Test beams at Fermilab will become regularly available once the Main Injector is completed (1999?), but only up to an energy of $120 \mathrm{GeV}$. Long-term studies requiring more beam time than pre-installation studies can be conducted only at a dedicated SSC facility.

For the reasons discussed above, there is clearly a need for an "as early as possible" beginning of the SSC MEB test beams program. However, it remains to discuss the effective and economical implementation of the upgrade to $2 \mathrm{TeV}$ test beams extracted from the HEB. The upgrade capability is specifically not precluded by HEB design or civil construction of the HEB, MEB, or MEB test beams facility. Because the $2 \mathrm{TeV}$ test beams program costs substantially more than the $200 \mathrm{GeV}$ program, its implementation was reduced to an upgrade option in order to reduce costs. Given the present budget uncertainties, it is not immediately obvious as to the best way to proceed, but there are benefits to be gained from the $2 \mathrm{TeV}$ program that are not achievable elsewhere.

The major purpose of the $2 \mathrm{TeV}$ test beams, as opposed to the $200 \mathrm{GeV}$ MEB test beams, would be to characterize the nonlinear response of the calorimeters. It is expected that a statistically significant sample of $\sim 10 \mathrm{TeV} / \mathrm{c}$ jets will be collected during a standard SSC year $\left(10 \mathrm{fb}^{-1}\right)$. Fragmentation products from such jets typically possess $20 \%$ of the jet momentum or $\sim 2 \mathrm{TeV} / \mathrm{c}$. A direct measurement of the single particle response of the calorimeter in the $2 \mathrm{TeV}$ energy range will reduce the energy scaling uncertainties that constrain the detector's ability to set limits on new phenomena. Thus, it is important to use test beams to understand calorimeter response at energies as high as possible. While the SSC physics program can begin without this extended coverage, it is the opinion of those physicists familiar with the SDC and GEM calorimeters that it is "highly desirable" that $2 \mathrm{TeV}$ test beams should be available early in the experimental program to ensure that the detectors can fulfill their scientific missions.

It should also be noted that, contrary to some speculation, it is not feasible or desirable to eliminate the $200 \mathrm{GeV}$ program in favor of the $2 \mathrm{TeV}$ program. The reasons for this are primarily that the $2 \mathrm{TeV}$ test beams will not be able to produce very low energy secondary particles (it may or may not be feasible to generate sufficient flux of low energy particles via a tertiary beam), and that availability of the $2 \mathrm{TeV}$ facility must wait for the completion of HEB commissioning. Since the GEM central tracker only needs energies provided by the MEB, but needs them at as early a date as possible, it makes much more sense to use the MEB test beams for central tracker testing. It should also be noted that an important increase in injector string operational experience is afforded by the test beams operations that also provide physics benefits to the detector collaborations.

Listed below are possible options together with approximate costs for completion of SSC test beam facilities.

1. Three $200 \mathrm{GeV}$ test beams (calibration hall used only for $200 \mathrm{GeV}$ beams) $\$ 35 \mathrm{M}$. 
2. Three $200 \mathrm{GeV}$ test beams with calibration hall that could later be used with $2 \mathrm{TeV}$ test beams (hereafter called a common calibration hall) $\$ 36.7 \mathrm{M}$.

3. Civil construction of two $2 \mathrm{TeV}$ beam lines, a $2 \mathrm{TeV}$ calibration hall, and outfit one beam line $\$ 85.6 \mathrm{M}$.

4. Same as number 3 but both $2 \mathrm{TeV}$ beam lines are outfitted $\$ 92.3 \mathrm{M}$

5. Three $200 \mathrm{GeV}$ test beams, one outfitted $2 \mathrm{TeV}$ beam line (civil construction completed for two), and a common calibration hall. $\$ 119.4 \mathrm{M}$

6. Three $200 \mathrm{GeV}$ test beams, two $2 \mathrm{TeV}$ test beams, and a common calibration hall. $\$ 126.5 \mathrm{M}$

7. Three $200 \mathrm{GeV}$ test beams, common calibration hall, and civil construction of two 2 TeV test beam lines. $\$ 50.3 \mathrm{M}$

Based on this information, plus the recognition that construction costs always increase with time, the best option with respect to physics goals and cost realities is option 7 . Thus, it is strongly recommended that:

1. The $200 \mathrm{GeV}$ Test Beam program (and completion of the MEB) proceed at a vigorous pace ensuring that $200 \mathrm{GeV}$ test beams are available a minimum of 4 years before collider commissioning, and

2. The conventional construction of the two $2 \mathrm{TeV}$ beam lines be completed at the same time as HEB conventional construction so that the upgrade to $2 \mathrm{TeV}$ test beams can proceed early in the SSC physics program with minimized additional cost,

3. If the SSC program is staged so that the injector complex is completed 5 years before the collider, then the $2 \mathrm{TeV}$ test beams should be brought into operation so that extensive detector studies can be conducted and experience with HEB operations can be gained that will enable very rapid progress in the physics program once the collider is commissioned (without the additional funds and detectors required by a fixed-target experimental program).

\subsection{Cost/Schedule Summary}

The cost changes to include a $2 \mathrm{TeV}$ fixed target facility comprise:

Element (w/o Contingency)

Increment for $2 \mathrm{TeV}$ FT
Cost (FY94 \$M)

$$
1,168
$$

The cost profile for this project is phased to complete the HEB Fixed Target Facility in FY98 and the $20 \times 20$ TeV Collider in FY04.

\subsection{References}

[1] "Proposal for Rephasing the Superconducting Super Collider, Revison A," Rainer Meinke, July 1993. 
Figure $\bar{i}, l$ Fluxes of $v$ and $\bar{v}$ and the associated background $\bar{v}$ and $v$ at UNK.

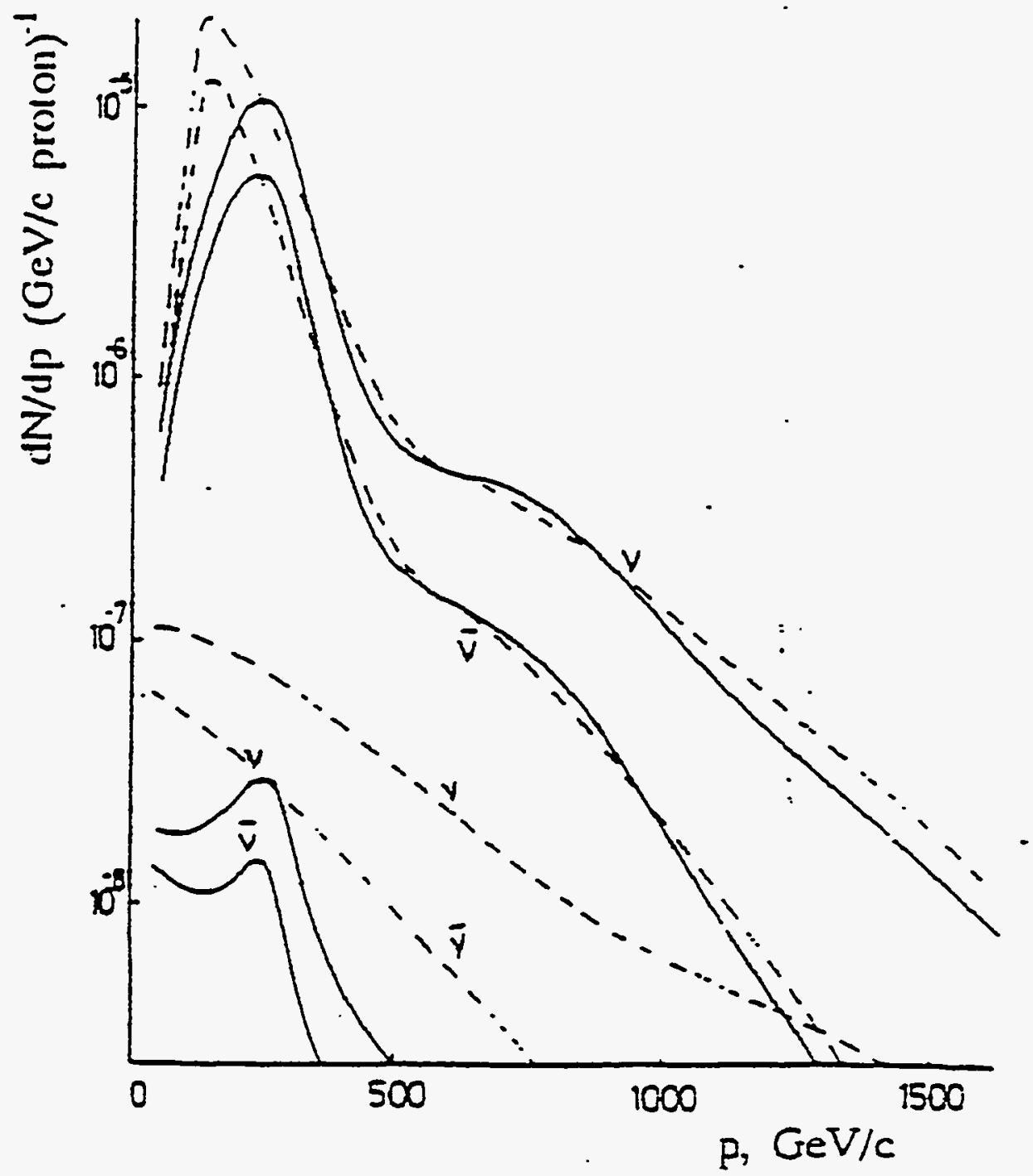


Figure 稳: Cross sections for $b \bar{b}$ duction as calculated by Berger and Meng. 11.1

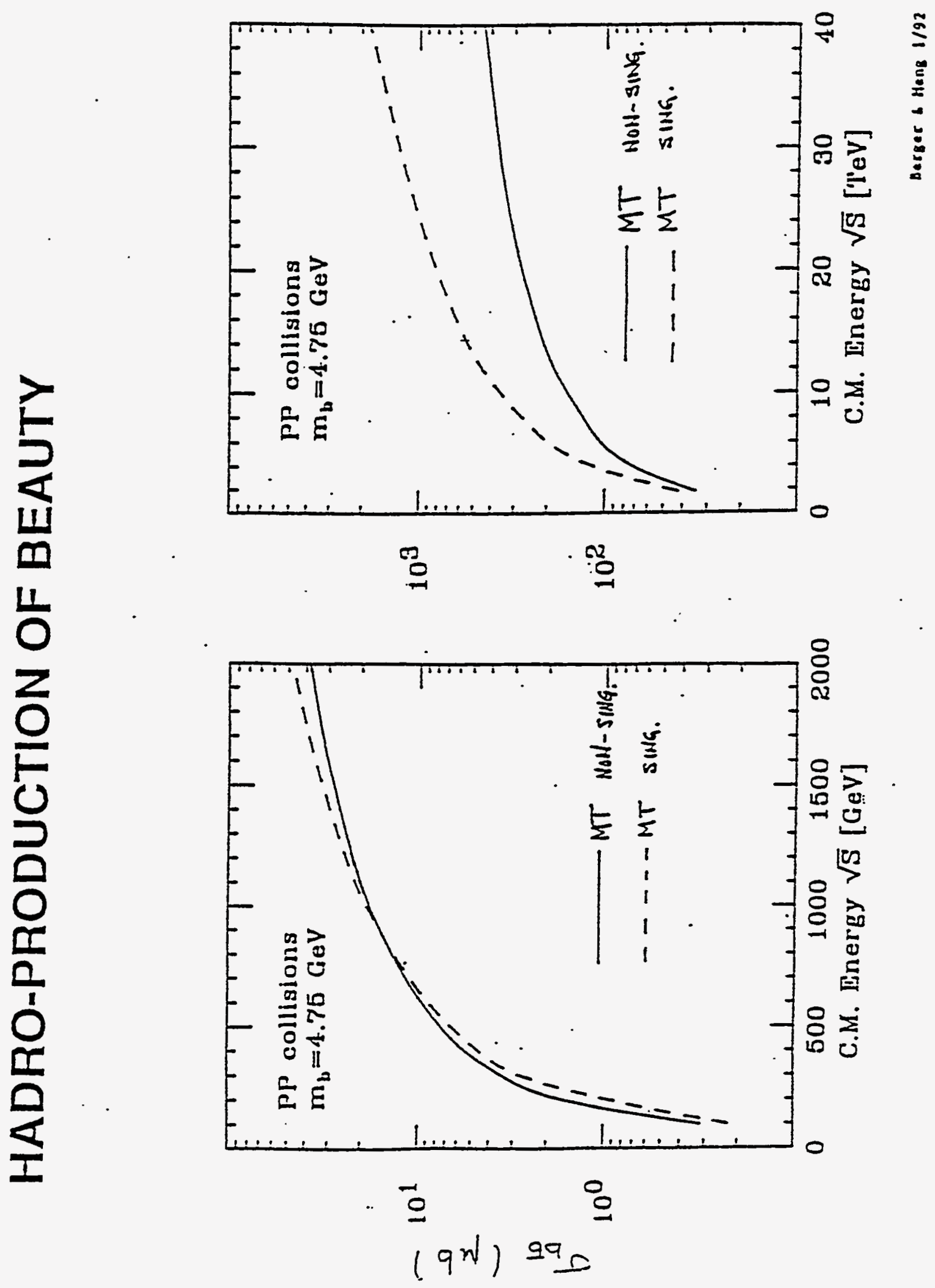




\section{Single collider ring with fixed target at $20 \mathrm{TeV}$}

\subsection{Introduction and Summary}

This section explores the physics opportunities for experiments using a single beam in the SSC Collider. This could provide physics results prior to the completion of the $20 \times 20 \mathrm{TeV}$ Collider. The experiments could be placed either in a straight section of the Collider exploiting internal targets or be placed behind an external target in a beam that is slowly extracted from the circulating proton beam. These experiments could continue during collider operation, with the HEB providing high intensity beams between collider fills and/or the collider providing protons at the level of $10^{8}$ protons/sec while beams are colliding and providing luminosity to the larger experiments. The use of the $20 \mathrm{TeV}$ ring as a dedicated fixed target machine with standard extraction systems is not being considered because of the very long fill times and slow ramp rate resulting in a poor duty cycle. However, it might be interesting to use the single turn extraction for the beam proton dump as a source for prompt neutrinos.

Physics topics that are being discussed for such experiments are B physics with emphasis on $\mathrm{CP}$ violating, and neutrino interactions, in particular $\tau$ neutrino interactions and deep inelastic scattering (DIS) at very high energies.

Direct competition for experiments at the HEB will be the fixed target program at Fermilab (1 $\mathrm{TeV})$ and at HERA $(0.8 \mathrm{TeV})$, and potentially UNK ( $3 \mathrm{TeV})$. The prime advantage of the Fermilab program is that it is fully implemented now, with extraction systems, neutrino beams, experiments halls and equipment. The new main injector will bring substantial increases in beam intensities. Still, with the primary focus at Fermilab on the Tevatron Collider, dedicated running time for the fixed target program will be limited to $30 \%$ or less over the next decade. Little is known for plans beyond the end of the decade. The HERA proton ring is available for parasitic use during the normal ep colliding beam operation. HELENA, an experiment to study CP

violation in the $\mathrm{B}-\rightarrow \Psi_{\mathrm{K}}$ transitions, is presently under consideration by the DESY management, with a decision expected later this fall. The future of UNK remains uncertain, its projected proton flux exceeds the HEB by an order of magnitude.

A potential $20 \mathrm{TeV}$ fixed target program has no competition on the energy front, though it will be limited in intensity if operated in parallel with the SSC collider. Its physics potential needs to be addressed in view of related information that will become available from experiments at other accelerators (Tevatron, LEP, HERA, SSC, LHC) over the next decade.

In summary, only a program that has an extremely compelling physics interest and for which the SSC beams provide a unique opportunity can justify the substantial costs for implementation of secondary beams, experimental facilities, and detectors, and operation at the SSC Laboratory. While B physics depends critically on the production rate (which can only be satisfied at the highest energies), most of the $\mathrm{v}$ physics goals could be addressed either at Fermilab after the completion of the main injector and an upgrade of the $\mathrm{v}$ beamline and also at UNK where a major fixed target facility is being planned.

\subsection{Beam parameters}

The relevant machine and beam parameters for extracted beam operation are listed in Table 9.1 for SSC injectors, HERA, the Tevatron, UNK and the SSC Collider with parasitic extraction. The average number of protons available are roughly equal for beams operating in the standard 
rapid cycling mode with extracted beams. Obviously, the secondary beam parameters will depend on the chosen physics program. At the SSC Collider, the protons are extracted from the stored beam by channeling in a mono-crystal. The proton rates are chosen such that $10 \%$ of the total circulating beam is extracted over the 22 hour storage cycle. This condition is imposed on the crystal extraction system so as to classify as parasitic beam usage when compared to the colliding beam experiments. The internal target experiment at HERA will operate under similar conditions, using $9 \%$ of the total beam over a period of 10 hours.

\subsection{Physics of Heavy Flavor Particles}

The prime interest in the physics of heavy flavors will remain their decays via the charged weak current. These studies will provide information on the elements of the CKM matrix, the form factors and decay constants. The study of $\mathrm{CP}$ violation in the $\mathrm{B}$ system is expected to provide the proof of direct CP violation in the weak decay amplitude as predicted by the standard model. Such studies will require much higher $B$ production rates and more sophisticated detectors and they are expected to remain a challenge during the next decade. While present fixed target experiments at the Tevatron are providing rates of more than 100,000 reconstructed charm decays, the number of detected $B$ decays remains very small. Over the next five to ten years, most of the information on the more common $\mathrm{B}$ decays is expected to come from $\mathrm{e}^{+} \mathrm{e}^{-}$colliders like CESR and LEP and from the Tevatron Collider experiments. It is expected that in the next few years these experiments will study the spectroscopy of beauty mesons and baryons. The measurement of the lifetimes of the individual beauty particles will be achieved with improved techniques and increasing statistics. Beyond these near term goals, the study of rare decays of B mesons and baryons will allow tests of the standard model predictions and thereby allow for the placement of strict limits on the presence of non-standard interactions.

Table 9.1: Beam for external fixed target experiments

\begin{tabular}{|c|c|c|c|c|c|c|}
\hline $\begin{array}{l}\text { Machine } \\
\text { units }\end{array}$ & $\begin{array}{l}\text { Energy } \\
{[\mathrm{GeV}]}\end{array}$ & \#bunches & $\begin{array}{c}\text { Protons } / \mathrm{b} \\
10^{10}\end{array}$ & $\begin{array}{c}\text { Spill } \\
\text { duration } \\
\text { [sec] }\end{array}$ & Duty cycle & $\begin{array}{l}\text { Average } \\
\text { rate } \\
\mathrm{p} / \mathrm{sec}\end{array}$ \\
\hline M.E.B. & 200 & 700 & 5 & 1 & $11 \%$ & $410^{12}$ \\
\hline HERA & 820 & 200 & 10 & 36,000 & $83 \%$ & $510^{7}$ \\
\hline Tevatron & 1,000 & 32 & 100 & 20 & $33 \%$ & $510^{11}$ \\
\hline H.E.B.* & 2,000 & 2,000 & 5 & 60 & $33 \%$ & $5.610^{11}$. \\
\hline UNK & 3,000 & $?$ & ? & 40 & $33 \%$ & $510^{12}$ \\
\hline S.S.C.** & 20,000 & 17,400 & 0.75 & 79,200 & $92 \%$ & $1.610^{8}$ \\
\hline
\end{tabular}

*extraction system and $2 \mathrm{TeV}$ transport not included in baseline.

$* *$ slow crystal extraction system not included in baseline, rates assume $10 \%$ beam extracted over 22 hours.

Figure 8.2 shows the calculated $\overline{\mathrm{b}}$ rate for $\mathrm{pp}$ and $\mathrm{p} \overline{\mathrm{p}}$ interactions as a function of c.m. energy. The two curves indicate the difference in the theoretical estimates based on QCD calculations by Berger and Meng1. At the Tevatron Collider the measured cross section is of the order $50 \mu \mathrm{b}$ or 0.001 of the total inelastic cross section, at SSC Collider energies the cross section is expected to increase to $1-2 \mathrm{mb}$ or about $1 \%$ of the total inelastic cross section. At the HEB beam energies, with a cm energy of $63 \mathrm{GeV}$, the $\bar{b}$ cross section is down to $30-50 \mathrm{nb}$, while a $20 \mathrm{TeV}$ beam 
results in a cm energy of $200 \mathrm{GeV}$ and a $b \bar{b}$ cross section as high as $2 \mu \mathrm{b}$ which constitutes $1 / 30,000$ of the interaction rate. These cross section are summarized in Table 9.2.

From present experience at fixed target experiments at the Tevatron, it is only too clear that higher energies are required to provide sufficient rate for the detection of $B$ hadron decays. It is also unlikely that an increase in $b \bar{b}$ production by a factor of three or four at the HEB will substantially change the outcome unless there is a substantial enhancement in the detector capabilities.

Table 9.2: $b \bar{b}$ cross sections and production rates

\begin{tabular}{lcccc}
\hline \hline & SSC Collider & SSC & Tevatron & SSC HEB \\
& F.T. & Collider & F.T. \\
\hline $\mathrm{E}_{\mathrm{cm}}(\mathrm{GeV})$ & 40,000 & 195 & 1,800 & 63 \\
Luminosity cm-2 $\mathrm{s}^{-1}$ & $10^{33}$ & $10^{7^{*}}$ & $510^{31}$ & $10^{7^{*}}$ \\
$\sigma_{\text {inel }}(\mathrm{mb})$ & 100 & 60 & 40 & 35 \\
$\sigma_{\mathrm{b} \overline{\mathrm{b}}}(\mu \mathrm{b})$ & 1,000 & 2 & 50 & $<0.04$ \\
$\mathrm{~N}_{\mathrm{b} \overline{\mathrm{b}} / \mathrm{sec}}$ & $10^{6}$ & $10^{3}$ & $510^{3}$ & $10^{2}$ \\
\hline \hline
\end{tabular}

*a maximum rate of $10^{7}$ interactions per sec is assumed.

Given the higher production rates at the Tevatron and SSC Colliders, the challenge for the measurement of rare decays remains the event selection, both at the level of the trigger as well as off-line. At present, only very simple trigger systems operate at rate of more than $10^{6} / \mathrm{s}$. The selection of $\mathrm{B}$ decays for the $\mathrm{CP}$ measurement can in principle rely on multiple leptons only, if one is prepared to restrict the measurement for decay modes containing a $\Psi \rightarrow 1+1^{-}$. At present, triggers rely on muons because the rejection of fake electrons appear to be very difficult. Similarly, present studies rely on muons for flavor tagging, even though substantial rate improvement could be obtained with electron and kaon tags. Other flavor tagging methods are still in the early phase of exploration, like the use of $\mathrm{B}^{* *}-\rightarrow \mathrm{B} \pi$ transitions. Precision vertex reconstruction could also lead to tags and improve the sample purity substantially. Furthermore, the measurement of time dependence is considered critical for the ultimate proof of $\mathrm{CP}$ violation. Presently planned upgrades of the Tevatron collider experiments include many new features and thereby enhance the sensitivity of these experiments for $\mathrm{CP}$ violation and other rare $\mathrm{B}$ decays.

A proposal under discussion at HERA might serve as a guide to what might be achievable in future fixed target experiments. The HERA-B group proposes the construction of a dedicated B experiment with a very demanding trigger system and an internal wire target system in the HERA proton beam. The goal of this experiment is to prove conclusively the existence of $\mathrm{CP}$ violation in $\mathrm{B}->\Psi \mathrm{K}$ decays. It is projected that a significant measurement of the $\mathrm{CP}$ asymmetry will require five years of operation with 8 wires targets and a data rate capability of $10^{8}$ interactions / $s$ to produce $40 \mathrm{~b} \bar{b}$ events / sec. This experiment also employs RICH counters to obtain flavor tags from kaons. 
A dedicated B experiment is being considered for the SSC with a crystal extracted secondary beam. A similar experiment could also be mounted downstream of an internal gas or wire target in the collider tunnel. The basic features of B experiments are given in Table 9.3 and they are compared with the projected performance of the upgraded CDF detector at the Tevatron. The principle advantage of the fixed target experiment is the larger acceptance, and the precise knowledge of the beam interaction point. In principle an active target could be employed to allow for the direct observation of the multiple vertices. In practice, small active targets of this kind have not performed to expectation, and it remains to be proven whether at higher energies a very high granularity silicon target would be a significant benefit.

Table 9.3: CP Asymmetry Measurements at the SSC

\begin{tabular}{lccccc}
\hline \hline & $\begin{array}{c}\text { SSC Fixed } \\
\text { Target }\end{array}$ & $\begin{array}{c}\text { SSC } \\
\text { Collider }\end{array}$ & $\begin{array}{c}\text { Hera B } \\
\ell \text { tag }\end{array}$ & $\begin{array}{c}\text { CDF } \\
\text { Upgrade }\end{array}$ & $\begin{array}{c}\text { B } \\
\text { Factory }\end{array}$ \\
\hline c.m. Energy $(\mathrm{GeV})$ & 195 & 40,000 & 40 & 1,800 & 10.6 \\
$\mathrm{~b} \overline{\mathrm{b}}$ cross section $\left(\mathrm{cm}^{2}\right)$ & $2 \cdot 10^{-30}$ & $10^{-27}$ & $2 \cdot 10^{-32}$ & $5 \cdot 10^{-29}$ & $1.1 \cdot 10^{-33}$ \\
Luminosity $\mathrm{cm}^{-2} \mathrm{~s}^{-1}$ & $5 \cdot 10^{32}$ & $10^{33}$ & $2 \cdot 10^{33}$ & $10^{32}$ & $3 \cdot 10^{33}$ \\
No. of $\mathrm{b} \overline{\mathrm{b}} / \mathrm{s}$ & $10^{3}$ & $10^{6}$ & 40 & $5 \cdot 10^{3}$ & 3.3 \\
$2 \mathrm{f}_{\mathrm{o}} \cdot \mathrm{x}$ & 0.75 & 0.75 & 1.5 & 1.5 & 2.2 \\
No. of B $\rightarrow \ell^{+} \ell^{-} \pi \pi / 10^{7} \mathrm{~s}$ & $1.4 \cdot 10^{5}$ & $1.4 \cdot 10^{8}$ & $1.1 \cdot 10^{4}$ & $1.4 \cdot 10^{6}$ & $1.4 \cdot 10^{3}$ \\
Overall Efficiency & 0.008 & $1.7 \cdot 10^{-5}$ & $8.3 \cdot 10^{-3}$ & $4 \cdot 10^{-4}$ & 0.23 \\
Tagged B Decays $/ 10^{7} \mathrm{~s}$ & 1,100 & 2,400 & 95 & 570 & 320 \\
Mixing Factor M & 0.75 & 0.47 & 0.77 & 0.50 & 0.53 \\
Dilution Factor D & 0.50 & 0.60 & 0.52 & 0.40 & 0.82 \\
$\Delta$ (sin 2B) & 0.08 & 0.07 & 0.26 & 0.21 & 0.13 \\
\hline \hline
\end{tabular}

$$
\begin{aligned}
& \mathrm{N}_{\text {prod }}\left(\Psi \mathrm{K}_{S}\right)=\int \mathrm{Ldt} \cdot \sigma \mathrm{b} \overline{\mathrm{b}} \cdot 2 \mathrm{f}_{\mathrm{O}} \cdot \mathrm{BR} \cdot \mathrm{x} \\
& \text { with } 2 \mathrm{f}_{\mathrm{O}}=0.75 \\
& \mathrm{BR}=\mathrm{BR}\left(\mathrm{B} \rightarrow \Psi \mathrm{K}_{\mathrm{S}}\right) \cdot \mathrm{BR}(\Psi \rightarrow \mu \mu) \cdot \mathrm{BR}\left(\mathrm{K}_{\mathrm{S}} \rightarrow \pi^{+} \pi^{-}\right) \\
& =4.10^{-4} \cdot 0.069 \cdot 0.686=1.9 \cdot 10^{-5}
\end{aligned}
$$

Both at the Tevatron Collider and the SSC $20 \mathrm{TeV}$ extracted beam significant $b \overline{\mathrm{b}}$ production rates are expected. The difference between experiments at these two machines will be more a question of the detector acceptance, trigger ability and particie identification that can be achieved given the high degree of sophistication required to perform a CP asymmetry measurement.

\subsection{Experimental Facilities and Detectors}

Costs for typical fixed target B experiments have been estimated to be in the range of $\$ 30 \mathrm{M}$ $40 \mathrm{M}$, no detailed designs are presently available. Typical neutrino experiments are comprised to several $1000 \mathrm{t}$ of target material followed by a magnetic spectrometer. In the past, costs for $\mathrm{v}$ experiments have been rather modest, but higher rates and finer granularity of $\tau$ detection are expected to substantially increase costs, to the level of probably $\$ 10 \mathrm{M}$ or more.

Internal Target B Experiments at $20 \mathrm{TeV}$ : Internal target experiments have the advantage that they do not require an extraction system, an external beam line, or a dedicated experimental hall, 
they can be placed in a long straight section of the accelerator close to an IP. In the Collider, the second beam pipe represents a limitation to the detector coverage, and a wider beam separation may be desirable. Gas targets have been operated successfully at CERN and other laboratories, they have the advantage that one can study interactions on hydrogen, though at the expense of lower luminosity. Heavier gases are contemplated for B experiments at LHC. Past experience has shown problems with background and shielding. The HERA B experiment uses a set of wire targets that operate in the halo of a single beam, this will require adjustments of the local $\beta$ function. Initial tests at HERA have produced encouraging results.

A typical internal target B experiment will require a hall of roughly $40 \mathrm{~m}$ length and $15 \mathrm{~m}$ width, which is estimated to cost a minimum of $\$ 3 \mathrm{M}$. If constructed underground, installation and access shafts will add about $\$ 2 \mathrm{M}$.

External Target $B$ experiments at $20 \mathrm{TeV}$ : An external target experiment will require a slow $(>20$ s) extraction system. Proposed B experiments at SSC and LHC foresee a crystal extraction system which is to be located in a long straight sections, preferentially sharing some of strong superconducting dipoles and shielding with the beam scraper system. Tests of the bent crystal system are under way at CERN and FNAL. Cost estimates for the complete system do not exist, but guesses places it in the $6 \mathrm{M} \$$ range, not including potential civil construction to extend the accelerator tunnel and to provide for an external beam line. The detector hall would be placed some $2000 \mathrm{~m}$ from the extraction point, its dimension should be roughly $90 \mathrm{~m}$ by $15 \mathrm{~m}$. It is not clear whethr such a hall could be located close to the surface, to avoid expensive excavation and access

\subsection{Summary}

Given the overall cost of the facilities for fixed target experiments at the SSC, it is very difficult if not impossible to justify a stand-alone program at the SSC Laboratory. A $20 \mathrm{TeV}$ external target B physics experiment is interesting and could potentially compete with collider B experiments. However, it would also only make operational sense to perform this experiment in a parasitic manner while other Collider experiments are proceeding.

\subsection{References:}

[1] E. Berger, T. Meng, Phys. Rev. 46 (1992) 169 


\section{Technical Advances}

\subsection{High-Field Magnet}

The question asked is prefaced by the following assumptions:

1. The basic magnet design stays the same or with only minor changes.

2. The accelerator (HEB) will operate more or less with the same characteristics.

3. The amount of rework or new design is minimized.

4. The degree of technical risk is minimal (already demonstrated performance).

If we look at the simple scaling rules that would be valid for such an option they would be:
a) Containment pressure $\sim \mathrm{B}^{2}$.
b) Flux return iron thickness $\sim B^{1 / 2}$.
c) Quench protection scales as stored energy $\sim \mathrm{B}^{2}$.
d) Synchrotron radiation $\sim B^{4}$ and finally...
e) The conductor super current density at $B(M A X) \sim B^{2}$.

A point of concern to be considered a factor is that the pressure at 10T (11T max.) is approximately $\sim 7000 \mathrm{psi}$. The yield point of even hardened copper is $\sim 10,000 \mathrm{psi}$. If we apply the same engineering margins for operation at $4.2 \mathrm{~K}$ to $1.8 \mathrm{~K}$ then the answer is an operational field of approximately of $9.0 \mathrm{~T}$ for the projected nominal temperature of $1.8 \mathrm{~K}$. Additional concerns would be pre-stress (as presently obtained) is marginal, the critical current is adequate, but the iron return yoke is probably thin (7\% saturation).

A prototype dipole was selected because it was the last of series of ASST prototypes to be tested to be operated in a superfluid environment. Although it experienced a couple of training quenches, it attained a quench current excess of $10 \mathrm{KA}$ resulting in a bore field of 9.5 Tesla. The operating temperature of $1.8 \mathrm{~K}$ has several features, a very high "effective" thermal conductance and superfluid properties versus lower Carnot efficiency. Although the field required is quite high, the current density margin is adequate. The cryostat for HEB should have a minimal heat load. The largest contributor to the load of the system will be the dynamic losses which will almost double in the lower temperature case, but due to the greatly enhanced heat transfer characteristics should be manageable. The conversion of the HEB refrigerators to the superfluid presents a problem. They were not set up for an efficient superfluid operation mode. Certainly there will be a loss in efficiency at the lower temperature. The question as to the correction coil adequacy for the machine cycle is probably most critical with respect to the quadrupole trims, and their ability to maintain proper dipole/quad track in the case of the highly saturated dipole approximately $7 \%$. If the correctors are only short sample limited in performance, however, this aspect should be OK. The chromaticity sextupole correctors will have a larger magnetization term to compensate for, but their lower operating temperature increased current density capabilities should be enough to compensate.

The correction coil that will have a sizable saturation effect is the closed orbit dipole corrector. So care must be exercised in installation accuracy, or we may have to go back in and move magnets.

The injection front porch will have a larger tune shift upon establishing the ramp due to the larger critical current magnetization currents that are able to contribute to that shift. At most, this requirement should only require a slowing down of the re-establishment of the ramp cycle after 
injection. The only other concern is that of the larger Lorentz forces and due to saturation the increased coil to coil interactions.

The question of whether we can protect the HEB dipole during quench can, of course, be simulated to first order. The temperatures in question are high not as high as melting solder, but probably high enough to shorten the lifetime of the devices. There has been specified a new style of heater in HEB which could go a long way into mitigating this problem but only prototypical measurements will ascertain it for sure. Voltages, of course, are unknown, but if the quenches are uniform they are probably o.k. as well.

The final line of such an upgrade would be an HEB peak energy of $2.71 \mathrm{Tev}$. If for some reason one or more of the assumptions is off base then probably $2.5 \mathrm{Tev}$ will be the final HEB peak energy.

\subsection{High Temperature Super Conductors}

The impact of high $\mathrm{T}_{\mathrm{c}}$ super conductors on the Collider design will be minimal. The semi-metal superconductor $\mathrm{Nb}_{3} \mathrm{Sn}$ was one of the first superconductors discovered, but, due to its non metallic nature, not successfully competed with malleable alloys like $\mathrm{NbTi}$ or $\mathrm{NbZr}$ for commercial applications. The inability for a non metal to take high levels of stress without degradation has made the device fabrication of accelerator components from these material very complicated and expensive. Even if these problems are addressed and solved, it is doubtful the system can operate successfully at an elevated temperature due to flux flow voltages. The present winding geometries would have to be greatly modified to take advantage of the two dimensional nature of the high " $\mathrm{T}_{\mathrm{c}}$ " materials, i.e., field/current orientation correct for optimum performance. The one "high $\mathrm{T}_{\mathfrak{C}}$ application" that appears to be possible is its use in power leads.

\subsection{Certified Pinning Centers in Super Conducting Fibers}

The most recent direction taken in increasing the performance of malleable super conductors (high $\mathrm{J}_{\mathrm{c}}$ "superconducting critical current density") is the application of artificial pinning centers. It is fairly straight forward to estimate the order of magnitude of the magnetic flux pinning structure to maximize the critical current. The flux pinning sites must be in an array with distances comparable to the fluxon density or greater. This approach has been highly successful, having achieved nearly $4 \times 10^{3} \mathrm{~A} / \mathrm{mm}^{2}$ at $5 \mathrm{~T}$ and $4.2 \mathrm{~K}$. The intermediate field work below $5 \mathrm{~T}$ has yielded positive results, but higher field gains have been more elusive.

\subsection{Cryogenic Refrigeration}

Advances in the technology of cryogenic refrigeration have been made in recent years, particularly in the improvement in overall efficiencies. Whereas about five years ago industry was quoting efficiencies of around 20\% Carnot, the RFP for the Surface refrigerators called for a minimum of $28 \%$. and the bidders were quite confident of achieving over $30 \%$ and perhaps as much as $32 \%$. This confidence is based on efficiencies demonstrated in plants delivered at HERA and more recently at CERN. Furthermore this improvement is achieved without an increase in capital cost. Therefore, although the main advantage gained from this advancement of technology is a decrease in operating costs there may actually be some reduction in first cost as well.

There are two other areas where some possibility of improvement may be hoped for, but both are longer term efforts which are unlikely to pay off during the construction of the SSC. The first of these is in improving the main helium gas compressors. About 50\% of the loss occurs here. 
While no specific activity is currently evident in The United States, it is recognized that there may be significant savings here. A more efficient compressor would drastically reduce the cooling requirements for the system saving both first costs and operating costs. The other area is to perfect a process innovation which cascades a high temperature Nitrogen system with a Helium refrigerator operating between $80 \mathrm{~K}$ and $4 \mathrm{~K}$. There are many difficult technological problems involved but the payoff if successful could be handsome. There is a certain amount of activity in both areas in Europe and Japan. 
Appendix A. Working Group Members

\author{
Baggett, Neal \\ Barton, Henry \\ Bird, Fred \\ Blocker, Craig \\ Burgett, William \\ Coles, Mark \\ Diwan, Milind \\ Dombeck, Tom \\ Dorenbosch, Jheroen \\ Elioff, Tom \\ Fukui, T. \\ Funk, Warren \\ Gannon, Jeff \\ Gerig, Rod \\ Gurd, Dave \\ Jayakumar, Jay \\ Johnson, David \\ Kandaswamy, J. \\ Kirk, Tom \\ Kraushaar, P. F. \\ Larson, D. J. \\ Lubatti, Henry \\ Luth, Vera \\ Lynch, Harvey \\ Maschke, Al \\ McAshan, Mike \\ McBride, Patricia \\ McFarlane, $\mathrm{K}$. \\ McGill, John \\ McInturff, $\mathrm{Al}$ \\ Meinke, Rainer \\ Mokhov, Nikolai \\ Mitselmakher, Gena \\ Mulholland, George \\ Paige, Frank \\ Pal, Trivan \\ Prichard, Ben \\ Siegrist, Jim \\ Takashima, $M$. \\ Tompkins, John \\ Turcotte, Marc \\ Webber, Bob \\ Weisend II, J. G. \\ Wienands, $U$. \\ Winje, Russ \\ Wojcicki, Stan \\ Womersley, John \\ Yarba, Victor \\ Yost, George
}


Appendix B. Assigned Section Editors

1. Pbar-P program at $20 \mathrm{TeV}$ on $20 \mathrm{TeV}$ (Ken McFarlane)

2. Missing Magnet Schemes for $10 \mathrm{TeV}$ on $10 \mathrm{TeV}$ (Victor Yarba)

3. Missing Magnet Coils for $15 \mathrm{TeV}$ on $15 \mathrm{TeV}$ (Victor yarba)

4. Asymmetric P-P for $2 \mathrm{TeV}$ on $20 \mathrm{TeV}$ (Guenakh Mitselmakher)

5. Asymmetric P-P for $200 \mathrm{GeV}$ on $2 \mathrm{TeV}$ (Dave Johnson)

6. Fixed Target program at 2 and $20 \mathrm{TeV}$ (Vera Luth)

7. Test Beam Program for Detector Development at $2 \mathrm{TeV}$ (William Burgett)

8. HEB P-P Collider Program at $2 \mathrm{TeV}$ on $2 \mathrm{TeV}$ at $10^{33}$ or $10^{34}$ Luminosity (Jim Siegrist)

9. Development of 9 to 10 Tesla Magnet (AlMcInturff)

10. Other technology considerations: Refrigerator Efficiency (Rao), HTSC (McInturff), Artificial Pinning Centers (McInturff), Detector subjects (Tom Kirk) 
Appendix C. The Text of the 1986 P-Bar-P Study Group's Conclusions

\author{
AN ASSESSMENT OF THE ANTIPROTON-PROTON OPTION \\ FOR THE SSC \\ pp Study Group \\ SSC Central Design Group* \\ c/o Lawrence Berkley Laboratory \\ Berkeley, California 94720 \\ May 1986 \\ *B.C. Barish, Caltech (Chairman) \\ A.Chao, SSC/CDG \\ M. Harrison, SSC/CDG - Fermilab \\ R. Hollebeek, Univ. of Pennsylvania \\ D.E. Johnson,SSC/CDG - Fermilab \\ R. Kass, SSC/CDG - Ohio State University \\ A. Minten, CERN \\ S.Peggs, SSC/CDG \\ A.Wrulich, SSC/CDG, LBL
}

Chapter 6

Discussions and Conclusions

In this study, we have assessed the anti-proton collider option for the SSC. In particular, we have compared a collider with the pp collider of performance, reliability, cost and physics potential. Our main conclusions are summarized below:

I. A luminosity of $10^{33} \mathrm{~cm}^{-2} \mathrm{~s}^{-1}$ for a pbar-p collider does not appear practical. This is primarily because of the bore of the main ring collider magnets must be increased in order to obtain adequate separation of the proton and antiproton beams. In addition, the production of enough antiprotons would require major technical advances. By contrast, the maximum luminosity of the proton proton collider is limited by the number of allowed events per crossing, which could be increased for some experiments.

II. A luminosity of $10^{32} \mathrm{~cm}^{-2} \mathrm{~s}^{-1}$ can reasonably be expected by extrapolating the technology of present $C E R N$ and Fermilab sources. Analyzing such a p $\bar{p}$ collider has the emphasis of our study.

1. We find that for most of the physics objectives, there is simply a loss of a factor of ten in rate compared to the pp collider. Processes involving weak or electromagnetic couplings and/or low mass scales favor the higher luminosity pp option. Event rates for hypothetical processes involving new gauge bosons or composite quarks and leptons coupled strongly to the qq annihilation process are roughly equal for the two options.

2. The best cost estimate of realizing such a collider is that it would cost approximately $\$ 242 \mathrm{M}$ less than a pp collider. Although only one ring is required, the added costs both of the larger bore single ring and of the $\mathrm{p}$ source and holding complex nullify much of the saving. 
3. We note that the pp collider costs would be somewhat reduced, by about $\$ 45 \mathrm{M}$, if a separate holding ring were not used or if it were demonstrated that a smaller separation of the proton and antiproton beams was sufficient in the main collider ring. However, neither of these possibilities appears justified.

III. Judging from the CERN experiences, it appears that pp is at least a factor of worse than pp in reliability of performance.

Finally, we note that we have not considered a low luminosity $\left(L=10^{30} \mathrm{~cm}^{-2} \mathrm{~s}^{-1}\right)$ machine without orbit separation. Such a collider would require neither a bright source nor a larger main collider bore, resulting in greater cost savings. Neither have we analyzed the site-specific p $\bar{p}$ option at Fermilab, where savings in both the proton injector and the $\bar{p}$ source might be realized. 